Report no. NA-06/10

\title{
Two-sided a posteriori error bounds for incompressible quasi-Newtonian flows
}

\author{
Stefano Berrone ${ }^{\mathrm{a}} \quad$ Endre Süli $^{\mathrm{b}}$
}

\begin{abstract}
We develop a posteriori upper and lower error bounds for mixed finite element approximations of a general family of steady, viscous, incompressible quasiNewtonian flows in a bounded Lipschitz domain $\Omega \subset \mathbb{R}^{d}$; the family includes degenerate models such as the power-law model, as well as non-degenerate ones such as the Carreau model. The unified theoretical framework developed herein yields residual-based a posteriori bounds which measure the error in the approximation of the velocity in the $\mathrm{W}^{1, r}(\Omega)$ norm and that of the pressure in the $\mathrm{L}^{r^{\prime}}(\Omega)$ norm, $1 / r+1 / r^{\prime}=1$.
\end{abstract}

Subject classifications: AMS(MOS): 65N30, 65N15, 65G99, 76D07, 76D99

Key words and phrases: finite element methods, a posteriori error estimates, non-Newtonian fluids

Oxford University Computing Laboratory

Numerical Analysis Group

Wolfson Building

Parks Road

Oxford, England OX1 3QD

June, 2006

aPolitecnico di Torino, Dipartimento di Matematica, corso Duca degli Abruzzi 24, I-10129 Torino, Italy

${ }^{\mathrm{b}}$ University of Oxford, Computing Laboratory, Parks Road, Oxford OX1 3QD, United Kingdom 


\section{Introduction}

The aim of this paper is to develop two-sided a posteriori error bounds for finite element approximations of constant-density, viscous, steady, incompressible quasi-Newtonian flows. Partial differential equations with nonlinearities of the kind considered herein arise in a number of application areas, including geophysical models of the lithosphere, as well as chemical engineering, particularly in the modelling of the flow of pastes and dies.

Suppose that $\Omega$ is a bounded Lipschitz domain in $\mathbb{R}^{d}, d \geq 2$, scaled so that $|\Omega|=1$, and, for $r \in(1, \infty)$, let $r^{\prime}=r /(r-1)$. The fluid, whose motion in $\Omega$ is due to an external body force $\mathbf{f} \in\left[\mathrm{L}^{r^{\prime}}(\Omega)\right]^{d}$, has velocity $\mathbf{u}$ and kinematic pressure $p$. For ease of exposition, $\mathbf{u}$ will be assumed to satisfy a homogeneous Dirichlet boundary condition; more general boundary conditions will be addressed in Remark 24 . The strong form of the governing equations is

$$
\begin{array}{rlrl}
-\nabla \cdot \sigma & =\mathbf{f}, & & \text { in } \Omega, \\
\nabla \cdot \mathbf{u}=0, & & \text { in } \Omega, \\
\mathbf{u} & =\mathbf{0}, & & \text { on } \partial \Omega=\Gamma,
\end{array}
$$

where

$$
\sigma(x, \mathbf{u})=k(x,|e(\mathbf{u})|) e(\mathbf{u})-p I
$$

is the stress tensor, $I$ denotes the $d \times d$ identity matrix, and the strain tensor $e(\mathbf{u}) \in \mathbb{R}_{\text {symm }}^{d \times d}$ is defined by its components

$$
e(\mathbf{u})_{i j}=\frac{1}{2}\left(\frac{\partial u_{i}}{\partial x_{j}}+\frac{\partial u_{j}}{\partial x_{i}}\right), \quad i, j=1, \ldots, d .
$$

Here $\mathbb{R}_{\text {symm }}^{d \times d}$ denotes the set of all symmetric real-valued $d \times d$ matrices. The function $k$ is the viscosity coefficient that characterizes the nonlinear viscosity model of the flow. Equation (1.1) is the momentum equation of the flow and (1.2) is the continuity equation in its usual form for a constant-density incompressible fluid.

The weak formulation of the boundary value problem requires finding the functions $\mathbf{u} \in \mathrm{V}$ and $p \in \mathrm{Q}$ such that

$$
\begin{aligned}
a(\mathbf{u}, \mathbf{v})+b(p, \mathbf{v}) & =(\mathbf{f}, \mathbf{v}) & & \forall \mathbf{v} \in \mathrm{V} \\
b(q, \mathbf{u}) & =0 & & \forall q \in \mathrm{Q},
\end{aligned}
$$

where $\mathrm{V}=\left[\mathrm{W}_{0}^{1, r}(\Omega)\right]^{d}, \mathrm{Q}=\mathrm{L}_{0}^{r^{\prime}}(\Omega)=\mathrm{L}^{r^{\prime}}(\Omega) / \mathbb{R}$,

$$
a(\mathbf{u}, \mathbf{v})=\int_{\Omega} k(x,|e(\mathbf{u})|) e(\mathbf{u}): e(\mathbf{v}) \mathrm{d} \Omega, \quad b(q, \mathbf{v})=-\int_{\Omega}(\nabla \cdot \mathbf{v}) q \mathrm{~d} \Omega .
$$

We shall adopt the following structural hypothesis on the viscosity coefficient.

Assumption $(A)$ : We assume that $k \in \mathrm{C}(\bar{\Omega} \times(0, \infty))$ and that, given $r \in(1, \infty)$ as above, there exist constants $\alpha \in[0,1]$ and $\varepsilon, K_{1}, K_{2}>0$ such that, for all $x \in \bar{\Omega}$, 


$$
\begin{aligned}
& K_{1}\left[(t+s)^{\alpha}(1+t+s)^{1-\alpha}\right]^{r-2}(t-s) \leq k(x, t) t-k(x, s) s \quad \text { for all } t \geq s>0 ; \\
& k(x, t) \leq K_{2}\left[t^{\alpha}(1+t)^{1-\alpha}\right]^{r-2} \quad \text { for all } t>0, \text { and } \\
& \quad|k(x, t) t-k(x, s) s| \leq K_{2}\left[(t+s)^{\alpha}(1+t+s)^{1-\alpha}\right]^{r-2}|t-s|
\end{aligned}
$$

for all $s, t>0$ satisfying $|(s / t)-1| \leq \varepsilon$.

For the sake of notational simplicity, we shall write $k(\cdot)$ instead of $k(x, \cdot)$. The parameter $\alpha$ in (A) measures the degree of singularity/degeneracy in $k(\cdot)$ for a given value of $r \in(1, \infty) \backslash\{2\}$ in the sense that the closer $\alpha$ is to 1 the more singular/degenerate $k(\cdot)$ is, for $r<2$, respectively $r>2$. When $r=2, t \mapsto k(t) t$ is uniformly monotone and globally Lipschitz continuous. For example:

(a) the power-law model with $k(t)=2 \mu t^{r-2}$ corresponds to $\alpha=1$; when $r=2$, this reduces to $k(t) \equiv 2 \mu$, yielding the Stokes equations which govern the stationary flow of a viscous incompressible Newtonian fluid;

(b) the Carreau law $k(t)=k_{\infty}+\left(k_{0}-k_{\infty}\right)\left(1+\lambda t^{2}\right)^{(\theta-2) / 2}$ with $k_{0}>k_{\infty} \geq 0, \lambda>0$, $\theta \in(1, \infty)$ corresponds to $\alpha=0$ with $r=\theta$ if $k_{\infty}=0$, and $r=2$ if $\theta \in(1,2]$ and $k_{\infty}>0$.

We equip the spaces $\mathrm{V}$ and $\mathrm{Q}$ with the norms

$$
\|\mathbf{v}\|_{\mathrm{V}}=\|e(\mathbf{v})\|_{\mathrm{L}^{r}(\Omega)} \quad \text { and } \quad\|q\|_{\mathrm{Q}}=\inf _{c \in \mathbb{R}}\|q+c\|_{\mathrm{L}^{r^{\prime}}(\Omega)},
$$

and recall from [1] that the bilinear form $b(\cdot, \cdot)$ satisfies the following inf-sup condition: there exists a positive constant $c_{0}$ such that

$$
\inf _{q \in \mathrm{Q}} \sup _{\mathbf{v} \in \mathrm{V}} \frac{b(q, \mathbf{v})}{\|q\|_{\mathrm{Q}}\|\mathbf{v}\|_{\mathrm{V}}} \geq c_{0} \quad \forall q \in \mathrm{Q} .
$$

In Section 2, we shall assume that the finite element subspaces $\mathrm{V}_{h}$ and $\mathrm{Q}_{h}$ of the spaces $\mathrm{V}$ and Q satisfy an analogous inf-sup condition, with inf-sup constant $c_{0}^{\prime}>0$.

The fact that $\|\cdot\|_{\mathrm{V}}$ is a norm on $\mathrm{V}$ is a consequence of Korn's inequality (cf. [16, 19]) which asserts the existence of a constant $C=C(r, d, \Omega), 1<r<\infty$, such that

$$
\|\mathbf{v}\|_{\mathrm{W}^{1, r}(\Omega)} \leq C\|e(\mathbf{v})\|_{\mathrm{L}^{r}(\Omega)} \quad \forall \mathbf{v} \in \mathrm{V} .
$$

In the sequel, for the sake of notational simplicity, we shall suppress the dependence of all constants on $r, d$ and $\Omega$; in particular, we shall write $C$ or $c$ instead of $C(r, d, \Omega)$.

The definition of the norm on $\mathrm{Q}$ reflects the fact that in the case of Dirichlet boundary condition on $\partial \Omega$ the pressure in the model is determined only up to an additive constant. Let $\mathrm{V}^{\prime}$ denote the dual space of $\mathrm{V}$ and let $\mathrm{Q}^{\prime}$ be the dual space of $\mathrm{Q}$; the spaces $\mathrm{V}^{\prime}$ and $\mathrm{Q}^{\prime}$ have the norms

$$
\|\mathbf{f}\|_{\mathrm{V}^{\prime}}=\sup _{\mathbf{v} \in \mathrm{V}} \frac{\langle\mathbf{f}, \mathbf{v}\rangle}{\|\mathbf{v}\|_{\mathrm{V}}} \quad \text { and } \quad\|g\|_{\mathrm{Q}^{\prime}}=\sup _{q \in \mathrm{Q}} \frac{\langle g, q\rangle}{\|q\|_{\mathrm{Q}}}
$$


Here, in the definition of $\|\cdot\|_{\mathrm{V}^{\prime}},\langle\cdot, \cdot\rangle$ denotes the duality pairing between $\mathrm{V}^{\prime}$ and $\mathrm{V}$, and in the definition of $\|\cdot\|_{\mathrm{Q}^{\prime}}$ it signifies the duality pairing between $\mathrm{Q}^{\prime}$ and $\mathrm{Q}$; as the choice of spaces over which the duality pairings act will always be clear from the context we have chosen not to indicate them explicitly in our notation $\langle\cdot, \cdot\rangle$.

Over the last decade, there has been considerable interest both in the mathematical analysis of quasi-Newtonian flow problems of this kind and in their finite element approximation. The existence and uniqueness of solutions to the boundary value problem (1.4), (1.5) was studied by Baranger and Najib [5] and Barrett and Liu [7]. In particular, it is known that (1.4), (1.5) has a unique solution $(\mathbf{u}, p) \in \mathrm{V} \times \mathrm{Q}$. Concerning the a priori error analysis of finite element methods for quasi-Newtonian flow equations, we refer to Baranger and Najib [5], Du and Gunzburger [14], Sandri [21], Barrett and Liu [7,8], Barrett and Bao [6], and Bao [3]. Baranger and El Amri [4] were the first to pursue the a posteriori error analysis of conforming finite element approximations to a quasi-Newtonian flow in the case of the Carreau law. Subsequently, Simms [23] considered the a posteriori error analysis of Fortin's element for conforming mixed finite element approximations of quasi-Newtonian flow problems and, more recently, Sandri [22] studied the a posteriori error analysis of conforming mixed finite element approximations of the power-law model and derived a posteriori upper bounds for the case of $1<r<2$. In fact, Sandri's upper bounds on the error in the velocity and the pressure will emerge from our analysis for the special case of $k(t)=2 \mu t^{r-2}, 1<r<2$; similarly, the upper bounds of Baranger and El Amri [4] are arrived at by selecting $k(t)=k_{\infty}+\left(k_{0}-k_{\infty}\right)\left(1+\lambda t^{2}\right)^{(\theta-2) / 2}, 1<\theta \leq 2$. For nonconforming finite element methods, Padra [20] derived a posteriori upper bounds for Fortin-Soulie [15] piecewise quadratic approximations of quasi-Newtonian flows. In the case of Carreau-type nonlinearities in two space dimensions, Bao and Barrett [6] developed a posteriori upper bounds based on the linear nonconforming element of Kouhia and Stenberg [18] which involves continuous piecewise linear approximation for one velocity component and a discontinuous linear Crouzeix-Raviart element for the other in tandem with piecewise constant approximation of the pressure. More recently, Carstensen and Funken [11] established two-sided a posteriori error bounds for quite a general class of conforming and nonconforming finite element methods for steady quasi-Newtonian flows, albeit under a stronger structural hypothesis than Assumption (A): the function $k$ was assumed to be uniformly monotone and uniformly Lipschitz-continuous, which corresponds to taking $r=2$ in Assumption (A). A posteriori upper bounds for the power-law model were also considered by Verfürth in [25], as an example of his general error analysis for finite element approximations of nonlinear variational problems.

The purpose of the present paper is to develop two-sided a posteriori error bounds, under Assumption (A), for (V,Q)-conforming finite element approximations to (1.4), (1.5), for the entire range of $r \in(1, \infty)$. A distinctive feature of problem $(1.4),(1.5)$ is that, in general, there is no value of $r>1$ other than $r=2$ such that the nonlinear differential operator is both uniformly monotone and uniformly Lipschitz-continuous in the Sobolev norm $\|\cdot\|_{W^{1, r}(\Omega)}$. Hence, following the work of Barrett and Liu [7,8], we shall rely here on uniform monotonicity and local Lipschitz continuity properties in Sobolev quasi-norms. Our a posteriori upper bounds first appeared, in a somewhat different 
form, in the unpublished technical report [9].

The paper is structured as follows. In Section 2 we state the finite element discretisation of the boundary-value problem. In Section 3, we establish some preliminary results which will then be used in Sections 4 and 5 to derive our a posteriori bounds on the error of the approximations $\mathbf{u}_{h}$ and $p_{h}$ in the norms $\|\cdot\|_{\mathrm{V}}$ and $\|\cdot\|_{\mathrm{Q}}$, respectively, in terms of residual functionals and computable finite element residuals. The main results of the paper are the following a posteriori upper and lower bounds on the error.

Theorem 1 Let $(\mathbf{u}, p) \in \mathrm{V} \times \mathrm{Q}$ denote the solution to (1.4), (1.5), and let $\left(\mathbf{u}_{h}, p_{h}\right) \in$ $\mathrm{V}_{h} \times \mathrm{Q}_{h}$ be its finite element approximation defined by (2.2), (2.3). Then, there exist positive constants $C$ and $c$ depending on $K_{1}, K_{2}, c_{0}, c_{0}^{\prime}, r,\|\mathbf{f}\|_{\mathrm{V}^{\prime}}$ such that

$$
\begin{gathered}
\left\|\mathbf{u}-\mathbf{u}_{h}\right\|_{\mathrm{V}}^{\mathrm{R}_{\mathrm{U}}}+\left\|p-p_{h}\right\|_{\mathrm{Q}}^{\mathrm{G}} \leq C\left(\left\|\mathbf{S}_{1}\right\|_{\mathrm{V}^{\prime}}^{\mathrm{R}_{\mathrm{U}}^{\prime}}+\left\|\mathrm{S}_{2}\right\|_{\mathrm{Q}^{\prime}}^{\mathrm{G}^{\prime}}\right), \\
c\left(\left\|\mathbf{S}_{1}\right\|_{\mathrm{V}^{\prime}}^{\mathrm{R}_{\mathrm{L}}^{\prime}}+\left\|\mathrm{S}_{2}\right\|_{\mathrm{Q}^{\prime}}^{\mathrm{G}^{\prime}}\right) \leq\left\|\mathbf{u}-\mathbf{u}_{h}\right\|_{\mathrm{V}}^{\mathrm{R}_{\mathrm{L}}}+\left\|p-p_{h}\right\|_{\mathrm{Q}}^{\mathrm{G}},
\end{gathered}
$$

where $\mathrm{S}_{1}$ and $\mathrm{S}_{2}$ are residual functionals which are computably bounded; further, $\mathrm{R}_{\mathrm{U}}=$ $\max \{r, 2\}, \mathrm{R}_{\mathrm{L}}=\min \{r, 2\}$, я $=\max \left\{r^{\prime}, 2\right\}$, where $1 / \mathrm{R}_{\mathrm{U}}+1 / \mathrm{R}_{\mathrm{U}}^{\prime}=1,1 / \mathrm{R}_{\mathrm{L}}+1 / \mathrm{R}_{\mathrm{L}}^{\prime}=1$, $1 / \mathrm{s}+1 / \mathrm{q}^{\prime}=1$ and $c_{0}^{\prime}$ is the constant from the discrete inf-sup condition.

We substantiate the phrase computably bounded in the previous theorem by further bounding $S_{1}$ and $S_{2}$ in terms of computable residuals to deduce the following result.

Theorem 2 Let $(\mathbf{u}, p) \in \mathrm{V} \times \mathrm{Q}$ denote the solution to (1.4), (1.5), and let $\left(\mathbf{u}_{h}, p_{h}\right) \in$ $\mathrm{V}_{h} \times \mathrm{Q}_{h}$ denote its finite element approximation defined by (2.2), (2.3). Then, there exist positive constants $C_{U}$ and $c_{L}$, depending on $K_{1}, K_{2}, c_{0}, c_{0}^{\prime}, r$ and $f$, such that

$$
\begin{aligned}
\left\|\mathbf{u}-\mathbf{u}_{h}\right\|_{V}^{\mathrm{R}_{U}}+ & \left\|p-p_{h}\right\|_{Q}^{\mathrm{G}} \leq C_{U}\left[\left(\sum_{T \in \mathcal{T}_{h}} h_{T}^{r^{\prime}}\left\|\Pi_{T} \mathbf{R}_{T}\left(\left[\mathbf{u}_{h}, p_{h}\right]\right)\right\|_{L^{r^{\prime}}(T)}^{r^{\prime}}\right)^{\frac{\mathrm{R}_{U}^{\prime}}{r^{\prime}}}\right. \\
& +\left(\sum_{E \in \mathcal{E}_{h, \Omega}} h_{E}\left\|\Pi_{E} \mathbf{J}_{E}\left(\left[\mathbf{u}_{h}, p_{h}\right]\right)\right\|_{L^{r^{\prime}}(E)}^{r^{\prime}}\right)^{\frac{\mathrm{R}_{U}^{\prime}}{r^{\prime}}}+\left\|\nabla \cdot \mathbf{u}_{h}\right\|_{L^{r}(\Omega)}^{\mathrm{g}^{\prime}} \\
& +\left(\sum_{T \in \mathcal{T}_{h}} h_{T}^{r^{\prime}}\left\|\mathbf{R}_{T}\left(\left[\mathbf{u}_{h}, p_{h}\right]\right)-\Pi_{T} \mathbf{R}_{T}\left(\left[\mathbf{u}_{h}, p_{h}\right]\right)\right\|_{L^{r^{\prime}}(T)}^{r^{\prime}}\right)^{\frac{\mathrm{R}_{U}^{\prime}}{r^{\prime}}} \\
& \left.+\left(\sum_{E \in \mathcal{E}_{h, \Omega}} h_{E}\left\|\mathbf{J}_{E}\left(\left[\mathbf{u}_{h}, p_{h}\right]\right)-\Pi_{E} \mathbf{J}_{E}\left(\left[\mathbf{u}_{h}, p_{h}\right]\right)\right\|_{L^{r^{\prime}}(E)}^{r^{\prime}}\right)^{\frac{\mathrm{R}_{U}^{\prime}}{r^{\prime}}}\right]
\end{aligned}
$$

and

$$
c_{L}\left[\left(\sum_{T \in \mathcal{T}_{h}} h_{T}^{r^{\prime}}\left\|\mathbf{R}_{T}\left(\left[\mathbf{u}_{h}, p_{h}\right]\right)\right\|_{L^{r^{\prime}}(T)}^{r^{\prime}}\right)^{\frac{\mathrm{R}_{L}^{\prime}}{r^{\prime}}}+\left(\sum_{E \in \mathcal{E}_{h, \Omega}} h_{E}\left\|\mathbf{J}_{E}\left(\left[\mathbf{u}_{h}, p_{h}\right]\right)\right\|_{L^{r^{\prime}}(E)}^{r^{\prime}}\right)^{\frac{\mathrm{R}_{L}^{\prime}}{r^{\prime}}}\right.
$$




$$
\begin{array}{r}
\left.+\left\|\nabla \cdot \mathbf{u}_{h}\right\|_{L^{r}(\Omega)}^{\mathrm{g}^{\prime}}\right] \leq\left\|\mathbf{u}-\mathbf{u}_{h}\right\|_{\mathrm{V}}^{\mathrm{R}_{\mathrm{L}}}+\left\|p-p_{h}\right\|_{Q}^{\mathrm{g}} \\
+\left(\sum_{T \in \mathcal{T}_{h}} h_{T}^{r^{\prime}}\left\|\mathbf{R}_{T}\left(\left[\mathbf{u}_{h}, p_{h}\right]\right)-\Pi_{T} \mathbf{R}_{T}\left(\left[\mathbf{u}_{h}, p_{h}\right]\right)\right\|_{L^{r^{\prime}(T)}}^{r^{\prime}}\right)^{\frac{\mathrm{R}_{\mathrm{L}}^{\prime}}{r^{\prime}}} \\
+\left(\sum_{E \in \mathcal{E}_{h, \Omega}} h_{E}\left\|\mathbf{J}_{E}\left(\left[\mathbf{u}_{h}, p_{h}\right]\right)-\Pi_{E} \mathbf{J}_{E}\left(\left[\mathbf{u}_{h}, p_{h}\right]\right)\right\|_{L^{r^{\prime}(E)}}^{r^{\prime}}\right)^{\frac{\mathrm{R}_{\mathrm{L}}^{\prime}}{r^{\prime}}}
\end{array}
$$

where $\mathbf{R}_{T}\left(\left[\mathbf{u}_{h}, p_{h}\right]\right)$ is the element-residual of the strong form of the momentum equation, $\mathbf{J}_{E}\left(\left[\mathbf{u}_{h}, p_{h}\right]\right)$ is the jump of the normal component of the stress tensor across an element-face $E$ in the triangulation, $\nabla \cdot \mathbf{u}_{h}$ is the residual of the continuity equation, and $\mathrm{R}_{\mathrm{U}}=\max \{r, 2\}, \mathrm{R}_{\mathrm{L}}=\min \{r, 2\}, \mathrm{g}=\max \left\{r^{\prime}, 2\right\}, 1 / \mathrm{R}_{\mathrm{U}}+1 / \mathrm{R}_{\mathrm{U}}^{\prime}=1,1 / \mathrm{R}_{\mathrm{L}}+1 / \mathrm{R}_{\mathrm{L}}^{\prime}=1$, $1 / \mathrm{s}+1 / \mathrm{s}^{\prime}=1$. Further, $\mathcal{E}_{h, \Omega}$ denotes the set of all element-faces internal to $\Omega$, and $\mathcal{T}_{h}$ is the set of all elements in the triangulation of $\Omega$.

Remark 3 We observe that $\mathrm{R}_{\mathrm{L}}=\mathrm{s}^{\prime}$, and therefore all the bounds can be expressed in terms of the two symbols: $\mathrm{R}_{\mathrm{U}}$ and $\mathrm{R}_{\mathrm{L}}$ and of their duals; nevertheless, we prefer to use different symbols for the powers of the pressure error and of $\mathrm{S}_{2}$ to stress the fact that this power is the same in the upper and the lower estimates.

In Section 6 we present some numerical results on uniform grids to investigate the robustness and effectivity of our error bounds. In Section 7 we implement the error bounds in a simple adaptive algorithm whose performance is exhibited on several model problems.

We adopt the following notational conventions.

Notation 4 When the letter $C$ appears in a mathematical formula without further qualifications, it is understood to mean a positive constant, independent of the discretization parameter $h$. For any $\xi, \eta>0: \xi \precsim \eta \Longleftrightarrow \exists C>0: \xi \leq C \eta$; $\xi \asymp \eta \Longleftrightarrow \xi \precsim \eta$ and $\eta \precsim \xi$.

\section{Finite element approximation}

For the sake of simplicity of presentation, we shall henceforth suppose that $\Omega \subset \mathbb{R}^{d}$ is a bounded polyhedral domain and that $\left\{\mathcal{T}_{h}\right\}_{h>0}$ is a shape-regular family of subdivisions of $\Omega$ consisting of $d$-dimensional open simplexes $T \in \mathcal{T}_{h}$, each of which is an affine image of the open unit simplex

$$
\hat{T}=\left\{\hat{x}=\left(\hat{x}_{1}, \ldots, \hat{x}_{d}\right) \in \mathbb{R}^{d}: 0<\hat{x}_{i}<1, i=1, \ldots, d, 0<\hat{x}_{1}+\ldots+\hat{x}_{d}<1\right\} .
$$

For any $T \in \mathcal{T}_{h}$ we denote by $\mathcal{E}(T)$ the set of its $(d-1)$-dimensional faces (for $d=2$ edges); we denote by $\mathcal{E}_{h}=\bigcup_{T \in T_{h}} \mathcal{E}(T)$ the set of all faces of $\mathcal{T}_{h}$. Moreover, we define $\mathcal{E}_{h, \Omega}=\left\{E \in \mathcal{E}_{h}: E \not \supset \partial \Omega\right\}$. 
Suppose that $\mathrm{V}_{h} \subset \mathrm{V}$ is a finite element space consisting of continuous piecewise polynomial $d$-component vector-functions defined on the triangulation $\mathcal{T}_{h}$ of $\Omega$ and $\mathrm{Q}_{h} \subset$ $\mathrm{Q}$ is a finite element space consisting of continuous or discontinuous piecewise polynomial functions defined on $\mathcal{T}_{h}$. We shall assume that the pair $\left(\mathrm{V}_{h}, \mathrm{Q}_{h}\right)$ satisfies the following inf-sup condition: there exists a positive constant $c_{0}^{\prime}$, independent of the discretisation parameter $h>0$, such that

$$
\inf _{q_{h} \in \mathrm{Q}_{h}} \sup _{\mathbf{v}_{h} \in \mathrm{V}_{h}} \frac{b\left(q_{h}, \mathbf{v}_{h}\right)}{\left\|q_{h}\right\|_{\mathrm{Q}}\left\|\mathbf{v}_{h}\right\|_{\mathrm{V}}} \geq c_{0}^{\prime} .
$$

The finite element approximation of our model problem has the following form: find $\mathbf{u}_{h} \in \mathrm{V}_{h}$ and $p_{h} \in \mathrm{Q}_{h}$ such that

$$
\begin{aligned}
a\left(\mathbf{u}_{h}, \mathbf{v}_{h}\right)+b\left(p_{h}, \mathbf{v}_{h}\right) & =\left(\mathbf{f}, \mathbf{v}_{h}\right) & & \forall \mathbf{v}_{h} \in \mathrm{V}_{h} \\
b\left(q_{h}, \mathbf{u}_{h}\right) & =0 & & \forall q_{h} \in \mathrm{Q}_{h} .
\end{aligned}
$$

Under the stated hypotheses problem $(2.2),(2.3)$ has a unique solution $\left(\mathbf{u}_{h}, p_{h}\right)$ in $\mathrm{V}_{h} \times \mathrm{Q}_{h}$ (cf. $[4,7])$.

For each $T \in \mathcal{T}_{h}$ and for each $E \in \mathcal{E}_{h}$ we define:

$$
\omega_{E}=\bigcup_{\left\{T^{\prime}: E \in \mathcal{E}\left(T^{\prime}\right)\right\}} T^{\prime}, \quad \tilde{\omega}_{T}=\bigcup_{\left\{T^{\prime}: \partial T \cap \partial T^{\prime} \neq \emptyset\right\}} T^{\prime}, \quad \tilde{\omega}_{E}=\bigcup_{\left\{T^{\prime}: E \cap \partial T^{\prime} \neq \emptyset\right\}} T^{\prime} .
$$

Note that the set $\omega_{E}$ is the union of $d$-dimensional elements that share the $(d-1)$ dimensional face $E$, whereas the sets $\tilde{\omega}_{T}$ and $\tilde{\omega}_{E}$ are unions of elements that share at least one point with $T$ or $E$, respectively.

For each $E \in \mathcal{E}_{h}$ we consider a unit vector $\hat{n}_{E}$ such that $\hat{n}_{E}$ is orthogonal to $E$. Given any $E \in \mathcal{E}_{h, \Omega}$ and any $\varphi$ with $\varphi_{\left.\right|_{T^{\prime}}} \in \mathrm{C}^{0}\left(T^{\prime}\right)$ for all $T^{\prime} \in \omega_{E}$, we denote by $\llbracket \varphi \rrbracket_{E}$ the jump of $\varphi$ across $E$ along the orientation of $\hat{n}_{E}$.

If the minimal angle of the family $\left\{\mathcal{T}_{h}\right\}_{h>0}$ is bounded away from zero, there exist constants, only dependent on the smallest angle in the triangulation, such that: $|T| \asymp$ $h_{T}^{d}, \forall T \in \mathcal{T}_{h}, h_{T} \asymp h_{E}, \forall E \in \mathcal{E}(T),|T| \asymp h_{E}^{d}, \forall T \in \omega_{E}$.

Let us denote by $\hat{E}$ the $(d-1)$-dimensional reference face, i.e. the face of $\hat{T}$ spanned by the vertices numbered $0, \ldots, d-1$. Moreover, let $\hat{b}_{\hat{T}}(\hat{x}, \hat{y})$ be the usual reference element bubble function and let $\hat{b}_{\hat{E}}$ be the usual reference face bubble function [26]. Let $\lambda_{i}, i=0, \ldots, d$, be the barycentric coordinates on the reference element; then, the reference element bubble function is $\hat{b}_{\hat{T}}=(d+1)^{d+1} \prod_{i=0, ., d} \lambda_{i}$, and the reference face bubble function is $\hat{b}_{\hat{E}}=d^{d} \prod_{i=0, \ldots, d-1} \lambda_{i}$; cf. [13,26].

Let $F_{T}: \hat{T} \rightarrow T$ be the invertible affine mapping that maps $\hat{T}$ onto $T$. We then define the element bubble function $b_{T}$ by $b_{T}=\hat{b}_{\hat{T}} \circ F_{T}^{-1}$.

Given any $E \in \mathcal{E}_{h, \Omega}$, let $T_{\sharp}$ and $T_{b}$ be the two elements of $\mathcal{T}_{h}$ such that $\omega_{E}=T_{\sharp} \cup T_{b}$; we enumerate the vertices of $T_{\sharp}$ and $T_{b}$ in such a way that the vertices of $E$ are numbered first. Let $T$ be one of the elements $T_{\sharp}$ and $T_{b}$, assume that $E$ has vertices $\mathbf{a}_{0}, \ldots, \mathbf{a}_{d-1}$ and denote by $\mathbf{a}_{c}$ the barycentre of the element $T$; let us partition $T$ into $d+1 d$-dimensional simplexes $T_{0}, \ldots, T_{d}$ with $T_{d}$ having $E$ as a face (see Figure 1 , right). Let $F_{E, T}: \hat{T} \rightarrow T_{d}$ 


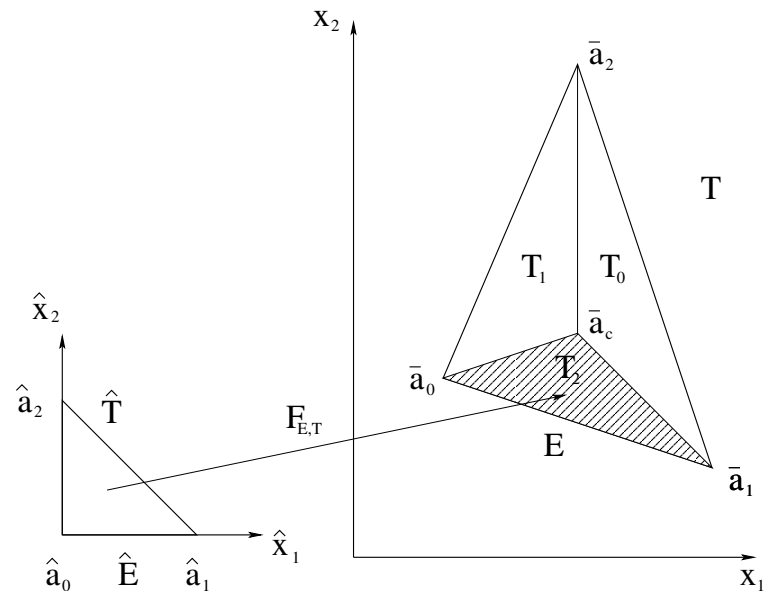

Figure 1: The mapping $F_{E, T}: \hat{T} \rightarrow T_{2}$

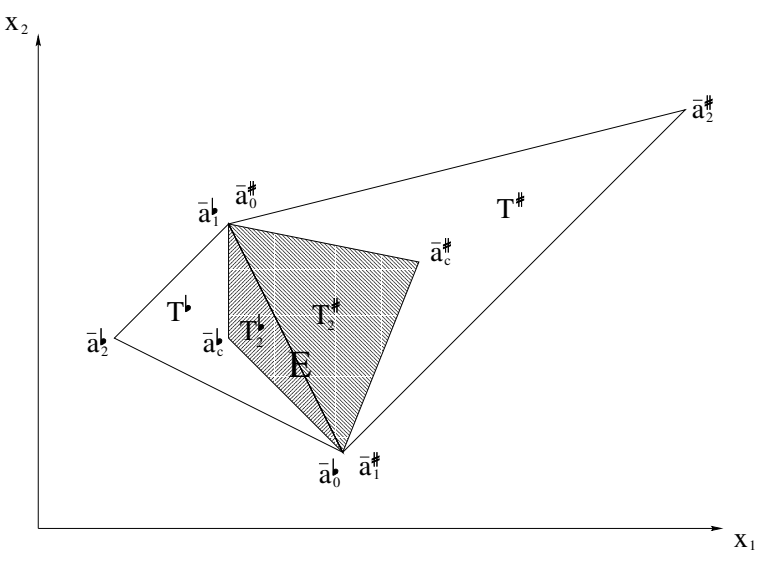

Figure 2: The support of the function $b_{E}$

be the invertible affine mapping that maps the reference element $\hat{T}$ onto the element $T_{d}$. Then, we define the face bubble function $b_{E}$ by gluing together the two bubble functions:

$$
b_{E, T_{\sharp}}=\hat{b}_{\hat{E}} \circ F_{E, T_{\sharp}}^{-1}, \quad b_{E, T_{b}}=\hat{b}_{\hat{E}} \circ F_{E, T_{b}}^{-1},
$$

each one being nonzero only on $T_{2}^{\sharp}$ and $T_{2}^{b}$, respectively. Finally, let us define the set $\grave{\omega}_{E}=T_{2}^{\sharp} \cup T_{2}^{b}$ (the shaded area in Figure 2, right). For the boundary face $E$ that belongs to the element $T$ only, we naturally identify $b_{E}$ with $b_{E, T}=\hat{b}_{\hat{E}} \circ F_{E, T}^{-1}$.

With this definition of face bubble functions we have a set of orthogonal functions, in the sense that the intersection of the supports of two different face bubble functions is the empty set. This property is also true for the set of element bubble functions.

Moreover, for the reference face $\hat{E}$ we define the extension operator $\hat{\mathcal{P}}_{\hat{E}}: \mathbb{P}_{i}(\hat{E}) \rightarrow$ $\mathbb{P}_{i}(\hat{T})$ which extends a polynomial of degree $i$ defined on the face $\hat{E}$ to a polynomial of the same degree defined on $\hat{T}$ with constant values along lines orthogonal to $\hat{E}$. Then, we define the extension operator $\mathcal{P}_{E}: \mathbb{P}_{i}(E) \rightarrow \mathbb{P}_{i}\left(\omega_{E}\right)$ which extends a polynomial of degree $i$ defined on the edge $E$ to a piecewise polynomial of the same degree defined on the patch $\dot{\omega}_{E}$ as follows:

$$
\left.\mathcal{P}_{E}(.)\right|_{T_{\sharp}}=\left.\hat{\mathcal{P}}_{\hat{E}}\left(.\left.\circ F_{T_{\sharp}}\right|_{\hat{E}}\right) \circ F_{T_{\sharp}}^{-1}\right|_{E} \quad \text { and }\left.\quad \mathcal{P}_{E}(.)\right|_{T_{b}}=\left.\hat{\mathcal{P}}_{\hat{E}}\left(.\left.\circ F_{T_{b}}\right|_{\hat{E}}\right) \circ F_{T_{b}}^{-1}\right|_{E} .
$$

The image of $\mathbb{P}_{i}(E)$ under the extension operator $\mathcal{P}_{E}$ is a subset of $\mathrm{C}^{0}\left(\grave{\omega}_{E}\right)$, but it is not contained in the function space $\mathrm{C}^{1}\left(\grave{\omega}_{E}\right)$.

In the following we shall have to collect all the $d$-dimensional simplexes belonging to

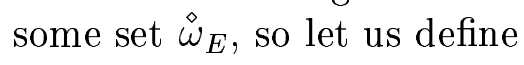

$$
\mathcal{T}_{h}^{\diamond}=\left\{T_{2} \in \grave{\omega}_{E}: E \in \mathcal{E}_{h, \Omega}\right\} .
$$

Besides, we denote by $I_{h}: \mathrm{V} \rightarrow \mathrm{V}_{h}$ the quasi-interpolation operator $[10,12,24]$ which possesses the following approximation properties. 
Lemma 5 Let $T \in \mathcal{T}_{h}$ and $E \in \mathcal{E}_{h}$ be arbitrary; then,

$$
\begin{aligned}
\left|v-I_{h} v\right|_{\mathrm{W}^{k, r}(T)} & \precsim h_{T}^{l-k}|v|_{\mathrm{W}^{l, r}\left(\tilde{\omega}_{T}\right)} \quad \forall v \in \mathrm{W}^{l, r}\left(\tilde{\omega}_{T}\right), \quad 0 \leq k \leq l \leq 1, \\
\left\|v-I_{h} v\right\|_{\mathrm{L}^{r}(E)} & \precsim h_{E}^{1-1 / r}|v|_{\mathrm{W}^{1, r}\left(\tilde{\omega}_{E}\right)} \quad \forall v \in \mathrm{W}^{1, r}\left(\tilde{\omega}_{E}\right),
\end{aligned}
$$

where the suppressed constants on the right-hand sides of these inequalities depend only on the smallest angle in the triangulation.

\section{Quasinorms in Sobolev spaces}

For $\alpha \in[0,1]$ and $t \in[0, \infty)$, we define

$$
\Xi_{\alpha}(t)=t^{\alpha}(1+t)^{1-\alpha}
$$

Hence,

$$
\Xi_{\alpha}^{\prime}(t)=(\alpha+t)(1+t)^{-\alpha} t^{\alpha-1} \quad \text { and } \quad \Xi_{\alpha}^{\prime \prime}(t)=-\alpha(1-\alpha) t^{\alpha-2}(1+t)^{-\alpha-1} .
$$

Therefore, for any $\alpha \in[0,1], t \mapsto \Xi_{\alpha}(t)$ is a strictly monotonic increasing function of $t \in[0, \infty)$; in particular, $t \mapsto \Xi_{0}(t)$ and $t \mapsto \Xi_{1}(t)$ are affine functions of $t \in[0, \infty)$. Furthermore, for $\alpha \in(0,1), t \mapsto \Xi_{\alpha}(t)$ is a strictly concave function of $t \in(0, \infty)$. The following Jensen-type inequality is easily proved by using Hölder's inequality and the triangle inequality in $\mathrm{L}^{r}(\Omega)$ : for any $r \in[1, \infty), \alpha \in[0,1]$, and all $w \in \mathrm{L}^{r}(\Omega)$,

$$
\left(\frac{1}{|\Omega|} \int_{\Omega}\left[\Xi_{\alpha}(|w(x)|)\right]^{r} \mathrm{~d} \Omega\right)^{\frac{1}{r}} \leq \Xi_{\alpha}\left(\left(\frac{1}{|\Omega|} \int_{\Omega}|w(x)|^{r} \mathrm{~d} \Omega\right)^{\frac{1}{r}}\right)
$$

According to our simplifying assumption from the start of the paper, $|\Omega|=1$; hence,

$$
\left\|\Xi_{\alpha}(|w|)\right\|_{L^{r}(\Omega)} \leq \Xi_{\alpha}\left(\|w\|_{L^{r}(\Omega)}\right)
$$

for all $r \in[1, \infty), \alpha \in[0,1]$, and all $w \in \mathrm{L}^{r}(\Omega)$.

We recall the following result from the paper of Barrett and Liu [7].

Lemma 6 Let $k$ satisfy assumption (A1) for $r \in(1, \infty)$ and $\alpha \in[0,1]$. Then, for all $M_{1}, M_{2}$ in $\mathbb{R}_{\mathrm{symm}}^{d \times d}$ and $\delta \geq 0$, we have that

$$
K_{1}\left[\Xi_{\alpha}\left(\left|M_{1}\right|+\left|M_{2}\right|\right)\right]^{r-2-\delta}\left|M_{1}-M_{2}\right|^{2+\delta} \leq\left(k\left(\left|M_{1}\right|\right) M_{1}-k\left(\left|M_{2}\right|\right) M_{2}\right):\left(M_{1}-M_{2}\right) .
$$

Let $k$ satisfy assumption (A2) for $r \in(1, \infty)$ and $\alpha \in[0,1]$. Then, for all $M_{1}, M_{2}$ in $\mathbb{R}_{\mathrm{symm}}^{d \times d}$ and $\delta \geq 0$, we have that

$$
\left|k\left(\left|M_{1}\right|\right) M_{1}-k\left(\left|M_{2}\right|\right) M_{2}\right| \leq K_{2}\left[\Xi_{\alpha}\left(\left|M_{1}\right|+\left|M_{2}\right|\right)\right]^{r-2+\delta}\left|M_{1}-M_{2}\right|^{1-\delta} .
$$

Next, we introduce the notation

$$
|\mathbf{v}|_{(\mathbf{w}, r, \alpha)}^{2}=\int_{\Omega}\left[\Xi_{\alpha}(|e(\mathbf{v})|+|e(\mathbf{w})|)\right]^{r-2}|e(\mathbf{v})|^{2} \mathrm{~d} \Omega, \quad \mathbf{v}, \mathbf{w} \in\left[\mathrm{W}^{1, r}(\Omega)\right]^{d}, 1<r<\infty .
$$


Proposition 7 Suppose that $r \in(1, \infty), \alpha \in[0,1]$ and $\mathbf{w} \in\left[\mathrm{W}^{1, r}(\Omega)\right]^{d}$; then, the following hold:

(i) $|\mathbf{v}|_{(\mathbf{w}, r, \alpha)} \geq 0$ for all $\mathbf{v} \in\left[\mathrm{W}^{1, r}(\Omega)\right]^{d}$. In particular, when $\mathbf{v} \in \mathrm{V}=\left[\mathrm{W}_{0}^{1, r}(\Omega)\right]^{d}$, $|\mathbf{v}|_{(\mathbf{w}, r, \alpha)}=0$ if, and only if, $\mathbf{v}=\mathbf{0}$;

(ii) (Quasi-triangle-inequality): there exists a constant $C=C(r)$ such that

$$
\left|\mathbf{v}_{1}+\mathbf{v}_{2}\right|_{(\mathbf{w}, r, \alpha)} \leq C\left(\left|\mathbf{v}_{1}\right|_{(\mathbf{w}, r, \alpha)}+\left|\mathbf{v}_{2}\right|_{(\mathbf{w}, r, \alpha)}\right)
$$

for all $\mathbf{v}_{1}, \mathbf{v}_{2} \in\left[\mathrm{W}^{1, r}(\Omega)\right]^{d}$;

(iii) For $1<r \leq 2$,

$$
|\mathbf{v}|_{(\mathbf{w}, r, \alpha)}^{2 / r} \leq\|e(\mathbf{v})\|_{L^{r}(\Omega)} \leq\left[\Xi_{\alpha}\left(\|e(\mathbf{v})\|_{L^{r}(\Omega)}+\|e(\mathbf{w})\|_{L^{r}(\Omega)}\right)\right]^{(2-r) / 2}|\mathbf{v}|_{(\mathbf{w}, r, \alpha)}
$$

for all $\mathbf{v} \in\left[\mathrm{W}^{1, r}(\Omega)\right]^{d}$.

For $2 \leq r<\infty$,

$$
\|e(\mathbf{v})\|_{L^{r}(\Omega)}^{r / 2} \leq|\mathbf{v}|_{(\mathbf{w}, r, \alpha)} \leq\left[\Xi_{\alpha}\left(\|e(\mathbf{v})\|_{L^{r}(\Omega)}+\|e(\mathbf{w})\|_{L^{r}(\Omega)}\right)\right]^{(r-2) / 2}\|e(\mathbf{v})\|_{L^{r}(\Omega)}
$$

for all $\mathbf{v} \in\left[\mathrm{W}^{1, r}(\Omega)\right]^{d}$.

Part (i) of this proposition follows from the definition of $|\cdot|_{(\mathbf{w}, r, \alpha)}$ and Korn's inequality. Part (ii) has been proved in the paper of Barrett and Liu [8]. The proof of (iii) is based on a straightforward application of Hölder's inequality and Jensen's inequality (3.1). Properties (i) and (ii) in Proposition 7 are the axioms of quasi-norm. Thus, for $\mathbf{w} \in \mathrm{V}=\left[\mathrm{W}^{1, r}(\Omega)\right]^{d},|\cdot|_{(\mathbf{w}, r, \alpha)}$ is a quasi-norm on $\mathrm{V}$. Property (iii) relates the Sobolev norm $\|\cdot\|_{\mathrm{V}}=|\cdot|_{\mathrm{W}^{1, r}(\Omega)}$ to the quasi-norm $|\cdot|_{(\mathbf{w}, r, \alpha)}$.

Now, we show the uniform monotonicity and local Lipschitz continuity of the semilinear form $a(\cdot, \cdot)$ with respect to the quasi-norm.

Lemma 8 Suppose that $r \in(1, \infty)$ and define the constants $C_{2}=2^{-|r-2|} K_{1}$ and $C_{3}=$ $2^{|r-2| / \max \left\{2, r^{\prime}\right\}} K_{2}$; then, for $i=1,2$, and all $\mathbf{v}_{1}, \mathbf{v}_{2}, \mathbf{w}$ in $\mathrm{V}$,

$$
\begin{gathered}
a\left(\mathbf{v}_{1}, \mathbf{v}_{1}-\mathbf{v}_{2}\right)-a\left(\mathbf{v}_{2}, \mathbf{v}_{1}-\mathbf{v}_{2}\right) \geq C_{2}\left|\mathbf{v}_{1}-\mathbf{v}_{2}\right|_{\left(\mathbf{v}_{i}, r, \alpha\right)}^{2} \\
\left|a\left(\mathbf{v}_{1}, \mathbf{w}\right)-a\left(\mathbf{v}_{2}, \mathbf{w}\right)\right| \leq C_{3}\left|\mathbf{v}_{1}-\mathbf{v}_{2}\right|_{\left(\mathbf{v}_{i}, r, \alpha\right)}^{\min \left\{1, \frac{2}{r^{\prime}}\right\}}\left[\Xi_{\alpha}\left(\left\|\mathbf{v}_{1}\right\|_{\mathrm{V}}+\left\|\mathbf{v}_{2}\right\|_{\mathrm{V}}\right)\right]^{\max \left\{0, \frac{r-2}{2}\right\}}\|\mathbf{w}\|_{\mathrm{V}}
\end{gathered}
$$

Proof. To prove (3.4), we use (3.2) with $\delta=0$, and $M_{i}=e\left(\mathbf{v}_{i}\right), i=1,2$. Hence, we deduce that, for any $\mathbf{v}_{1}, \mathbf{v}_{2} \in \mathrm{V}$,

$$
\begin{aligned}
& a\left(\mathbf{v}_{1}, \mathbf{v}_{1}-\mathbf{v}_{2}\right)-a\left(\mathbf{v}_{2}, \mathbf{v}_{1}-\mathbf{v}_{2}\right) \\
& \quad \geq K_{1} \int_{\Omega}\left|e\left(\mathbf{v}_{1}\right)-e\left(\mathbf{v}_{2}\right)\right|^{2}\left[\Xi_{\alpha}\left(\left|e\left(\mathbf{v}_{1}\right)\right|+\left|e\left(\mathbf{v}_{2}\right)\right|\right)\right]^{r-2} \mathrm{~d} \Omega .
\end{aligned}
$$


We note that, for $i=1,2$,

$$
\frac{1}{2}\left(\left|e\left(\mathbf{v}_{1}-\mathbf{v}_{2}\right)\right|+\left|e\left(\mathbf{v}_{i}\right)\right|\right) \leq\left|e\left(\mathbf{v}_{1}\right)\right|+\left|e\left(\mathbf{v}_{2}\right)\right| \leq 2\left(\left|e\left(\mathbf{v}_{1}-\mathbf{v}_{2}\right)\right|+\left|e\left(\mathbf{v}_{i}\right)\right|\right) .
$$

Suppose that $1<r \leq 2$; then, (3.6), the second inequality in (3.7) and the definition of the quasi-norm $|\cdot|_{\left(\mathbf{v}_{i}, r, \alpha\right)}$ imply that

$$
a\left(\mathbf{v}_{1}, \mathbf{v}_{1}-\mathbf{v}_{2}\right)-a\left(\mathbf{v}_{2}, \mathbf{v}_{1}-\mathbf{v}_{2}\right) \geq 2^{r-2} K_{1}\left|\mathbf{v}_{1}-\mathbf{v}_{2}\right|_{\left(\mathbf{v}_{i}, r, \alpha\right)}^{2}, \quad i=1,2,
$$

and hence (3.4) with $C_{2}=2^{r-2} K_{1}$ for $1<r \leq 2$.

Similarly, when $2 \leq r<\infty$, the first inequality in (3.7) and the definition of the quasi-norm $|\cdot|_{\left(\mathbf{v}_{i}, r, \alpha\right)}$ imply that

$$
a\left(\mathbf{v}_{1}, \mathbf{v}_{1}-\mathbf{v}_{2}\right)-a\left(\mathbf{v}_{2}, \mathbf{v}_{1}-\mathbf{v}_{2}\right) \geq 2^{2-r} K_{1}\left|\mathbf{v}_{1}-\mathbf{v}_{2}\right|_{\left(\mathbf{v}_{i}, r, \alpha\right)}^{2}, \quad i=1,2,
$$

and hence (3.4) with $C_{2}=2^{2-r} K_{1}$ for $2 \leq r<\infty$.

To show (3.5), we apply Hölder's inequality, the fact that $\|e(\mathbf{w})\|_{\mathrm{L}^{r}(\Omega)}=\|\mathbf{w}\|_{\mathrm{V}}$, and the inequality (3.2) with $M_{i}=e\left(\mathbf{v}_{i}\right), i=1,2$; hence, we deduce that

$$
\begin{aligned}
& \left|a\left(\mathbf{v}_{1}, \mathbf{w}\right)-a\left(\mathbf{v}_{2}, \mathbf{w}\right)\right|=\int_{\Omega}\left(k\left(\left|e\left(\mathbf{v}_{1}\right)\right|\right) e\left(\mathbf{v}_{1}\right)-k\left(\left|e\left(\mathbf{v}_{2}\right)\right|\right) e\left(\mathbf{v}_{2}\right)\right): e(\mathbf{w}) \mathrm{d} \Omega \\
& \leq\left(\int_{\Omega}\left|k\left(\left|e\left(\mathbf{v}_{1}\right)\right|\right) e\left(\mathbf{v}_{1}\right)-k\left(\left|e\left(\mathbf{v}_{2}\right)\right|\right) e\left(\mathbf{v}_{2}\right)\right|^{r^{\prime}} \mathrm{d} \Omega\right)^{1 / r^{\prime}}\|\mathbf{w}\|_{\mathrm{V}} \\
& \leq K_{2}\left(\int_{\Omega}\left|e\left(\mathbf{v}_{1}\right)-e\left(\mathbf{v}_{2}\right)\right|^{(1-\delta) r^{\prime}}\left[\Xi_{\alpha}\left(\left|e\left(\mathbf{v}_{1}\right)\right|+\left|e\left(\mathbf{v}_{2}\right)\right|\right)\right]^{(r-2+\delta) r^{\prime}} \mathrm{d} \Omega\right)^{1 / r^{\prime}}\|\mathbf{w}\|_{\mathrm{V}}
\end{aligned}
$$

Let $1<r \leq 2$ and define $\delta=1-\left(2 / r^{\prime}\right)$; then $(1-\delta) r^{\prime}=2,(r-2+\delta) r^{\prime}=r-2$. Therefore, using the first inequality in (3.7),

$$
\left|a\left(\mathbf{v}_{1}, \mathbf{w}\right)-a\left(\mathbf{v}_{2}, \mathbf{w}\right)\right| \leq 2^{(2-r) / r^{\prime}} K_{2}\left|\mathbf{v}_{1}-\mathbf{v}_{2}\right|_{\left(\mathbf{v}_{i}, r, \alpha\right)}^{2 / r^{\prime}}\|\mathbf{w}\|_{\mathrm{V}}, \quad i=1,2
$$

which is (3.5) with $C_{3}=2^{(2-r) / r^{\prime}} K_{2}$ for $1<r \leq 2$.

Now, let $2<r \leq \infty$ and hence $r^{\prime}=r /(r-1) \in(1,2)$; we shall use Hölder's inequality in the integral on the right-hand side of (3.8). Thus, we take $\delta=0$, split

$$
\left[\Xi_{\alpha}\left(\left|e\left(\mathbf{v}_{1}\right)\right|+\left|e\left(\mathbf{v}_{2}\right)\right|\right)\right]^{(r-2) r^{\prime}}=\left[\Xi_{\alpha}\left(\left|e\left(\mathbf{v}_{1}\right)\right|+\left|e\left(\mathbf{v}_{2}\right)\right|\right)\right]^{\frac{(r-2) r^{\prime}}{2}}\left[\Xi_{\alpha}\left(\left|e\left(\mathbf{v}_{1}\right)\right|+\left|e\left(\mathbf{v}_{2}\right)\right|\right)\right]^{\frac{(r-2) r^{\prime}}{2}}
$$

and group the first factor on the right with $\left|e\left(\mathbf{v}_{1}\right)-e\left(\mathbf{v}_{2}\right)\right|^{r^{\prime}}$. The application of Hölder's inequality with exponents $\beta=2 / r^{\prime}$ and $\beta^{\prime}=2 /\left(2-r^{\prime}\right), 1 / \beta+1 / \beta^{\prime}=1$, corresponding to the factors

$$
\left|e\left(\mathbf{v}_{1}\right)-e\left(\mathbf{v}_{2}\right)\right|^{r^{\prime}}\left[\Xi_{\alpha}\left(\left|e\left(\mathbf{v}_{1}\right)\right|+\left|e\left(\mathbf{v}_{2}\right)\right|\right)\right]^{(r-2) r^{\prime} / 2} \text { and }\left[\Xi_{\alpha}\left(\left|e\left(\mathbf{v}_{1}\right)\right|+\left|e\left(\mathbf{v}_{2}\right)\right|\right)\right]^{(r-2) r^{\prime} / 2},
$$

respectively, yields

$$
\begin{aligned}
\left|a\left(\mathbf{v}_{1}, \mathbf{w}\right)-a\left(\mathbf{v}_{2}, \mathbf{w}\right)\right| \leq & K_{2}\left(\int_{\Omega}\left|e\left(\mathbf{v}_{1}\right)-e\left(\mathbf{v}_{2}\right)\right|^{2}\left[\Xi_{\alpha}\left(\left|e\left(\mathbf{v}_{1}\right)\right|+\left|e\left(\mathbf{v}_{2}\right)\right|\right)\right]^{r-2} \mathrm{~d} \Omega\right)^{1 / 2} \\
& \times\left(\int_{\Omega}\left[\Xi_{\alpha}\left(\left|e\left(\mathbf{v}_{1}\right)\right|+\left|e\left(\mathbf{v}_{2}\right)\right|\right)\right]^{\frac{(r-2) r^{\prime}}{2} \cdot \frac{2}{2-r^{\prime}} \mathrm{d} \Omega}\right)^{\left(2-r^{\prime}\right) /\left(2 r^{\prime}\right)}\|\mathbf{w}\|_{\mathrm{v}}
\end{aligned}
$$


As $\frac{(r-2) r^{\prime}}{2} \cdot \frac{2}{2-r^{\prime}}=r, \frac{2-r^{\prime}}{2 r^{\prime}}=\frac{r-2}{2 r}$, using the second inequality in (3.7) we get that

$$
\left|a\left(\mathbf{v}_{1}, \mathbf{w}\right)-a\left(\mathbf{v}_{2}, \mathbf{w}\right)\right| \leq C_{3}\left|\mathbf{v}_{1}-\mathbf{v}_{2}\right|_{\left(\mathbf{v}_{i}, r, \alpha\right)}\left\|\left[\Xi_{\alpha}\left(\left|e\left(\mathbf{v}_{1}\right)\right|+\left|e\left(\mathbf{v}_{2}\right)\right|\right)\right]\right\|_{L^{r}(\Omega)}^{(r-2) / 2}\|\mathbf{w}\|_{\mathrm{V}}
$$

for all $\mathbf{v}_{1}, \mathbf{v}_{2}$ in $\mathrm{V}$, with $C_{3}=2^{(r-2) / 2} K_{2}$. On noting (3.1), the triangle inequality for the $\|\cdot\|_{L^{r}(\Omega)}$ norm, and that $t \mapsto \Xi_{\alpha}(t)$ is monotonic increasing, we have (3.5) for $2<r<\infty$.

Next, we show that the solutions to problems (1.4), (1.5) and (2.2), (2.3) can be bounded in terms of $\|\mathbf{f}\|_{\mathrm{V}^{\prime}}$.

Lemma 9 Let $(\mathbf{u}, p) \in \mathrm{V} \times \mathrm{Q}$ and $\left(\mathbf{u}_{h}, p_{h}\right) \in \mathrm{V}_{h} \times \mathrm{Q}_{h}$ denote the solutions to problems (1.4), (1.5) and (2.2), (2.3), respectively, and let $r \in(1, \infty)$; then

$$
\begin{gathered}
\|\mathbf{u}\|_{\mathrm{V}} \leq G^{-1}\left(\frac{1}{K_{1}}\|\mathbf{f}\|_{\mathrm{V}^{\prime}}\right), \quad\|p\|_{\mathrm{Q}} \leq \frac{1}{c_{0}}\left(\|\mathbf{f}\|_{\mathrm{V}^{\prime}}+K_{2}\left(H \circ G^{-1}\right)\left(\frac{1}{K_{1}}\|\mathbf{f}\|_{\mathrm{V}^{\prime}}\right)\right) \\
\left\|\mathbf{u}_{h}\right\|_{\mathrm{V}} \leq G^{-1}\left(\frac{1}{K_{1}}\|\mathbf{f}\|_{\mathrm{V}^{\prime}}\right), \quad\left\|p_{h}\right\|_{\mathrm{Q}} \leq \frac{1}{c_{0}^{\prime}}\left(\|\mathbf{f}\|_{\mathrm{V}^{\prime}}+K_{2}\left(H \circ G^{-1}\right)\left(\frac{1}{K_{1}}\|\mathbf{f}\|_{\mathrm{V}^{\prime}}\right)\right)
\end{gathered}
$$

where $c_{0}$ and $c_{0}^{\prime}$ are the inf-sup constants from (1.6) and (2.1), respectively, and $G$ and $H$ are continuous strictly monotonic increasing functions defined on $[0, \infty)$.

Proof. Taking $\mathbf{v}=\mathbf{u}$ in (1.4) and using (1.5), we have that

$$
a(\mathbf{u}, \mathbf{u})=(\mathbf{f}, \mathbf{u})=\langle\mathbf{f}, \mathbf{u}\rangle \leq\|\mathbf{f}\|_{\mathrm{V}^{\prime}}\|\mathbf{u}\|_{\mathrm{V}}
$$

Now, using (3.2) with $M_{1}=e(\mathbf{u}), M_{2}=0, \delta=0$ and (iii) of Proposition 7 with $\mathbf{w}=\mathbf{0}$, we obtain

$$
a(\mathbf{u}, \mathbf{u}) \geq K_{1}|\mathbf{u}|_{(\mathbf{0}, r, \alpha)}^{2} \geq K_{1}\|\mathbf{u}\|_{\mathrm{V}} G\left(\|\mathbf{u}\|_{\mathrm{V}}\right) ; \quad G(t)= \begin{cases}t \cdot\left[\Xi_{\alpha}(t)\right]^{r-2}, & 1<r \leq 2 \\ t^{r-1}, & r \geq 2\end{cases}
$$

Since $G: t \mapsto G(t)$ is continuous and strictly monotonic increasing on $[0, \infty)$, its inverse function $G^{-1}$ is continuous and strictly monotonic increasing on $[0, \infty)$. Hence,

$$
\|\mathbf{u}\|_{\mathrm{V}} \leq G^{-1}\left(\frac{1}{K_{1}}\|\mathbf{f}\|_{\mathrm{V}^{\prime}}\right)
$$

To bound $\|p\|_{\mathrm{Q}}$, note that, by the inf-sup condition (1.6),

$$
c_{0}\|p\|_{\mathrm{Q}} \leq \sup _{\mathbf{v} \in \mathrm{V}} \frac{b(p, \mathbf{v})}{\|\mathbf{v}\|_{\mathrm{V}}} .
$$

On the other hand, from (1.4), and using using (3.3) with $M_{1}=e(\mathbf{u}), M_{2}=0, \delta=0$, and (iii) of Proposition 7 with $\mathbf{w}=\mathbf{0}$, we obtain

$$
b(p, \mathbf{v})=(\mathbf{f}, \mathbf{v})-a(\mathbf{u}, \mathbf{v})=\langle\mathbf{f}, \mathbf{v}\rangle-a(\mathbf{u}, \mathbf{v}) \leq\|\mathbf{f}\|_{\mathrm{V}^{\prime}}\|\mathbf{v}\|_{\mathrm{V}}+K_{2} H\left(\|\mathbf{u}\|_{\mathrm{V}}\right)\|\mathbf{v}\|_{\mathrm{V}}
$$

where

$$
H(t)= \begin{cases}t^{r-1}, & 1<r \leq 2, \\ t \cdot\left[\Xi_{\alpha}(t)\right]^{r-2}, & 2 \leq r,\end{cases}
$$


and therefore,

$$
c_{0}\|p\|_{\mathrm{Q}} \leq\|\mathbf{f}\|_{\mathrm{V}^{\prime}}+K_{2} H\left(\|\mathbf{u}\|_{\mathrm{V}}\right)
$$

Clearly, $H: t \mapsto H(t)$ is continuous and strictly monotonic increasing on $[0, \infty)$. Together with our earlier bound $\|\mathbf{u}\|_{\mathrm{V}} \leq G^{-1}\left(\frac{1}{K_{1}}\|\mathbf{f}\|_{\mathrm{V}^{\prime}}\right)$, this proves (3.9); the proof of (3.10) is identical, except that (2.1) is used instead of (1.6).

Remark $10 A s|(\mathbf{f}, \mathbf{w})|=|\langle\mathbf{f}, \mathbf{w}\rangle| \leq\|\mathbf{f}\|_{\mathrm{V}^{\prime}}\|\mathbf{w}\|_{\mathrm{V}}$ and, by Hölder's inequality, $|a(\mathbf{v}, \mathbf{w})| \leq$ $K_{2} H\left(\|\mathbf{v}\|_{\mathrm{V}}\right)\|\mathbf{w}\|_{\mathrm{V}}$ and $|b(q, \mathbf{w})| \leq\|q\|_{\mathrm{Q}}\|\mathbf{w}\|_{\mathrm{V}}$, it follows, using (3.10), that

$$
\left|(\mathbf{f}, \mathbf{w})-a\left(\mathbf{u}_{h}, \mathbf{w}\right)-b\left(p_{h}, \mathbf{w}\right)\right| \leq\left(1+\frac{1}{c_{0}^{\prime}}\right)\left(\|\mathbf{f}\|_{\mathrm{V}^{\prime}}+K_{2}\left(H \circ G^{-1}\right)\left(\frac{1}{K_{1}}\|\mathbf{f}\|_{\mathrm{V}^{\prime}}\right)\right)\|\mathbf{w}\|_{\mathrm{V}}
$$

Hence, for $\mathbf{f} \in\left[\mathrm{L}^{r^{\prime}}(\Omega)\right]^{d}$ fixed, and the corresponding unique solution $\left(\mathbf{u}_{h}, p_{h}\right)$ of (2.2), (2.3) in $\mathrm{V}_{h} \times \mathrm{Q}_{h} \subset \mathrm{V} \times \mathrm{Q}$ thereby also fixed, $\mathbf{w} \mapsto(\mathbf{f}, \mathbf{w})-a\left(\mathbf{u}_{h}, \mathbf{w}\right)-b\left(p_{h}, \mathbf{w}\right)$ is a bounded (and therefore continuous) linear functional on $\mathrm{V}$; as such, it belongs to $\mathrm{V}^{\prime}$. It is this element of $\mathrm{V}^{\prime}$ that we denote by $\mathbf{S}_{1}$. Similarly, for $\mathbf{u}_{h} \in \mathrm{V}_{h} \subset \mathrm{V}$ fixed, $q \mapsto b\left(q, \mathbf{u}_{h}\right)$ is a bounded (and therefore continuous) linear functional on $\mathrm{Q}$, and as such, it belongs to $\mathrm{Q}^{\prime}$; it is this element of $\mathrm{Q}^{\prime}$ that we denote by $\mathrm{S}_{2}$.

Using $\mathbf{v}_{1}=\mathbf{u}$ and $\mathbf{v}_{2}=\mathbf{u}_{h}$ in (3.4) and (3.5), together with the bounds on $\|\mathbf{u}\|_{\mathrm{V}}$ and $\left\|\mathbf{u}_{h}\right\|_{\mathrm{V}}$ from Lemma 9 , we obtain the following result.

Lemma 11 Let $(\mathbf{u}, p) \in \mathrm{V} \times \mathrm{Q}$ and $\left(\mathbf{u}_{h}, p_{h}\right) \in \mathrm{V}_{h} \times \mathrm{Q}_{h}$ denote the solutions to problems (1.4), (1.5) and (2.2), (2.3), respectively, and suppose that $r \in(1, \infty)$; then,

$$
\begin{aligned}
& a\left(\mathbf{u}, \mathbf{u}-\mathbf{u}_{h}\right)-a\left(\mathbf{u}_{h}, \mathbf{u}-\mathbf{u}_{h}\right) \geq C_{2}\left|\mathbf{u}-\mathbf{u}_{h}\right|_{(\mathbf{u}, r, \alpha)}^{2} \\
& \quad\left|a(\mathbf{u}, \mathbf{w})-a\left(\mathbf{u}_{h}, \mathbf{w}\right)\right| \leq C_{4}\left|\mathbf{u}-\mathbf{u}_{h}\right|_{(\mathbf{u}, r, \alpha)}^{\min \left\{1, \frac{2}{r}\right\}}\|\mathbf{w}\|_{\mathrm{V}},
\end{aligned}
$$

where $C_{4}=C_{3} 2^{\max \left\{0, \frac{r-2}{2}\right\}}\left[\left(\Xi_{\alpha} \circ G^{-1}\right)\left(\frac{1}{K_{1}}\|\mathbf{f}\|_{\mathrm{V}^{\prime}}\right)\right]^{\max \left\{0, \frac{r-2}{2}\right\}}$, and $C_{2}=C_{2}\left(K_{1}, r\right), C_{3}=$ $\mathrm{C}_{3}\left(K_{2}, r\right)$ are as in Lemma 8 .

\section{The a posteriori error upper bound}

We begin by bounding the norms $\left\|\mathbf{u}-\mathbf{u}_{h}\right\|_{\mathrm{V}}$ and $\left\|p-p_{h}\right\|_{\mathrm{Q}}$ of the error in the approximations to the velocity and the pressure by the norms of the residual functionals $\mathbf{S}_{1} \in \mathrm{V}^{\prime}$ and $\mathrm{S}_{2} \in \mathrm{Q}^{\prime}$ defined below. We shall complete the a posteriori error analysis by showing that $\left\|\mathbf{S}_{1}\right\|_{\mathrm{V}^{\prime}}$ and $\left\|\mathrm{S}_{2}\right\|_{\mathrm{Q}^{\prime}}$ can, in turn, be bounded in terms of computable residuals. 


\subsection{Upper bound on the error in terms of residual functionals}

We define $\mathbf{S}_{1} \in \mathrm{V}^{\prime}$ by

$$
\left\langle\mathbf{S}_{1}, \mathbf{w}\right\rangle=(\mathbf{f}, \mathbf{w})-a\left(\mathbf{u}_{h}, \mathbf{w}\right)-b\left(p_{h}, \mathbf{w}\right) \quad \forall \mathbf{w} \in \mathrm{V}
$$

Similarly, we define $\mathrm{S}_{2} \in \mathrm{Q}^{\prime}$ by

$$
\left\langle\mathrm{S}_{2}, q\right\rangle=-b\left(q, \mathbf{u}_{h}\right) \quad \forall q \in \mathrm{Q} .
$$

The existence of the functionals $S_{1}$ and $S_{2}$ as elements of $V^{\prime}$ and $Q^{\prime}$, respectively, is the consequence of Remark 10 following Lemma 9.

For $1<r<\infty$ the reflexive Banach space $\mathrm{V}$ is continuously and densely embedded into the reflexive Banach space $\left[\mathrm{L}^{r}(\Omega)\right]^{d}$. Hence, $\left[\mathrm{L}^{r^{\prime}}(\Omega)\right]^{d}$, the dual space of $\left[\mathrm{L}^{r}(\Omega)\right]^{d}$, is continuously and densely embedded into $\mathrm{V}^{\prime}$. In particular, $\mathbf{f} \in\left[\mathrm{L}^{r^{\prime}}(\Omega)\right]^{d}$ can be identified with an element of $\mathrm{V}^{\prime}$ (also denoted $\mathbf{f}$ for the sake of notational simplicity) via $\langle\mathbf{f}, \mathbf{w}\rangle=(\mathbf{f}, \mathbf{w})$ for all $\mathbf{w} \in \mathrm{V}$. Hence, the definitions (4.1) and (4.2) imply that

$$
\begin{aligned}
a\left(\mathbf{u}_{h}, \mathbf{w}\right)+b\left(p_{h}, \mathbf{w}\right) & =\left\langle\mathbf{f}-\mathbf{S}_{1}, \mathbf{w}\right\rangle & & \forall \mathbf{w} \in \mathrm{V} \\
b\left(q, \mathbf{u}_{h}\right) & =\left\langle-\mathrm{S}_{2}, q\right\rangle & & \forall q \in \mathrm{Q} .
\end{aligned}
$$

On subtracting these from (1.4) and (1.5), respectively, we obtain

$$
\begin{array}{rlrl}
a(\mathbf{u}, \mathbf{w})-a\left(\mathbf{u}_{h}, \mathbf{w}\right)+b\left(p-p_{h}, \mathbf{w}\right) & =\left\langle\mathbf{S}_{1}, \mathbf{w}\right\rangle & & \forall \mathbf{w} \in \mathrm{V}, \\
b\left(q, \mathbf{u}-\mathbf{u}_{h}\right) & =\left\langle\mathrm{S}_{2}, q\right\rangle & \forall q \in \mathrm{Q} .
\end{array}
$$

Proposition 12 Let $(\mathbf{u}, p) \in \mathrm{V} \times \mathrm{Q}$ denote the solution to (1.4), (1.5), and let $\left(\mathbf{u}_{h}, p_{h}\right) \in$ $\mathrm{V}_{h} \times \mathrm{Q}_{h}$ denote its finite element approximation defined by (2.2), (2.3); then, there exists a positive constant $C=C\left(K_{1}, K_{2}, c_{0}, r,\|\mathbf{f}\|_{\mathrm{V}^{\prime}}\right)$ such that

$$
\begin{aligned}
\left|\mathbf{u}-\mathbf{u}_{h}\right|_{(\mathbf{u}, r, \alpha)}^{2} & \leq C\left(\left\|\mathbf{S}_{1}\right\|_{\mathrm{V}^{\prime}}^{\mathrm{R}_{\mathrm{U}}^{\prime}}+\left\|\mathrm{S}_{2}\right\|_{\mathrm{Q}^{\prime}}^{\mathrm{g}^{\prime}}+\left\|\mathbf{S}_{1}\right\|_{\mathrm{V}^{\prime}}\left\|\mathrm{S}_{2}\right\|_{\mathrm{Q}^{\prime}}\right) \\
\left\|p-p_{h}\right\|_{\mathrm{Q}}^{\mathrm{g}} & \leq C\left(\left\|\mathbf{S}_{1}\right\|_{\mathrm{V}^{\prime}}^{\mathrm{g}}+\left|\mathbf{u}-\mathbf{u}_{h}\right|_{(\mathbf{u}, r, \alpha)}^{2}\right)
\end{aligned}
$$

where $\mathrm{R}_{\mathrm{U}}=\max \{r, 2\}$, я $=\max \left\{r^{\prime}, 2\right\}, 1 / \mathrm{R}_{\mathrm{U}}+1 / \mathrm{R}_{\mathrm{U}}^{\prime}=1,1 /$ я $+1 / \mathrm{g}^{\prime}=1$.

Proof. According to the inf-sup condition (1.6), identity (4.3), the definition of the norm $\|\cdot\|_{\mathrm{V}^{\prime}}$ and (3.12), we have that

$$
c_{0}\left\|p-p_{h}\right\|_{\mathrm{Q}} \leq\left\|\mathbf{S}_{1}\right\|_{\mathrm{V}^{\prime}}+C_{4}\left|\mathbf{u}-\mathbf{u}_{h}\right|_{(\mathbf{u}, r, \alpha)}^{\min \left\{1, \frac{2}{r}\right\}},
$$

and hence (4.6) on noting that $\min \left\{1, \frac{2}{r^{\prime}}\right\}=2 \min \left\{\frac{1}{2}, \frac{1}{r^{\prime}}\right\}=2 /$ s.

On the other hand, taking $\mathbf{w}=\mathbf{u}-\mathbf{u}_{h}$ in (4.3), then using (4.4) with $q=p-p_{h}$, (3.11), and the definitions of the norms $\|\cdot\|_{\mathrm{V}^{\prime}}$ and $\|\cdot\|_{\mathrm{Q}^{\prime}}$, we get that

$$
C_{2}\left|\mathbf{u}-\mathbf{u}_{h}\right|_{(\mathbf{u}, r, \alpha)}^{2} \leq\left\|\mathbf{S}_{1}\right\|_{\mathrm{V}^{\prime}}\left\|\mathbf{u}-\mathbf{u}_{h}\right\|_{\mathrm{V}}+\left\|\mathrm{S}_{2}\right\|_{\mathrm{Q}^{\prime}}\left\|p-p_{h}\right\|_{\mathrm{Q}}
$$


Multiplying (4.8) by $c_{0}$ and then eliminating $c_{0}\left\|p-p_{h}\right\|_{\mathrm{Q}}$ using (4.7) gives

$$
\begin{aligned}
c_{0} C_{2}\left|\mathbf{u}-\mathbf{u}_{h}\right|_{(\mathbf{u}, r, \alpha)}^{2} \leq & c_{0}\left\|\mathbf{S}_{1}\right\|_{\mathrm{V}^{\prime}}\left\|\mathbf{u}-\mathbf{u}_{h}\right\|_{\mathrm{V}}+\left\|\mathbf{S}_{1}\right\|_{\mathrm{V}^{\prime}}\left\|\mathrm{S}_{2}\right\|_{\mathrm{Q}^{\prime}} \\
& +C_{4}\left\|\mathrm{~S}_{2}\right\|_{\mathrm{Q}^{\prime}}\left|\mathbf{u}-\mathbf{u}_{h}\right|_{(\mathbf{u}, r, \alpha)}^{\min \left\{1, \frac{2}{r^{\prime}}\right\}} .
\end{aligned}
$$

Part (iii) of Proposition 7, with $\mathbf{v}=\mathbf{u}-\mathbf{u}_{h}$ and $\mathbf{w}=\mathbf{u}$ implies that

$$
\left\|\mathbf{u}-\mathbf{u}_{h}\right\|_{\mathrm{V}} \leq\left[\Xi_{\alpha}\left(\left\|\mathbf{u}-\mathbf{u}_{h}\right\|_{\mathrm{V}}+\|\mathbf{u}\|_{\mathrm{V}}\right)\right]^{\max \left\{0, \frac{2-r}{2}\right\}}\left|\mathbf{u}-\mathbf{u}_{h}\right|_{(\mathbf{u}, r, \alpha)}^{\min \left\{1, \frac{2}{r}\right\}}
$$

Also, recalling from Lemma 9 that

$$
\|\mathbf{u}\|_{\mathrm{V}} \leq G^{-1}\left(\frac{1}{K_{1}}\|\mathbf{f}\|_{\mathrm{V}^{\prime}}\right) \quad \text { and } \quad\left\|\mathbf{u}_{h}\right\|_{\mathrm{V}} \leq G^{-1}\left(\frac{1}{K_{1}}\|\mathbf{f}\|_{\mathrm{V}^{\prime}}\right)
$$

gives

$$
\left\|\mathbf{u}-\mathbf{u}_{h}\right\|_{\mathrm{V}}+\|\mathbf{u}\|_{\mathrm{V}} \leq 2\left(\|\mathbf{u}\|_{\mathrm{V}}+\left\|\mathbf{u}_{h}\right\|_{\mathrm{V}}\right) \leq 4 G^{-1}\left(\frac{1}{K_{1}}\|\mathbf{f}\|_{\mathrm{V}^{\prime}}\right) .
$$

Hence, (4.10) and the fact that $t \mapsto \Xi_{\alpha}(t)$ is monotonic increasing on $[0, \infty)$ imply that

$$
\left\|\mathbf{u}-\mathbf{u}_{h}\right\|_{\mathrm{V}} \leq 2^{\max \{0,2-r\}}\left[\left(\Xi_{\alpha} \circ G^{-1}\right)\left(\frac{1}{K_{1}}\|\mathbf{f}\|_{\mathrm{V}^{\prime}}\right)\right]^{\max \left\{0, \frac{2-r}{2}\right\}}\left|\mathbf{u}-\mathbf{u}_{h}\right|_{(\mathbf{u}, r, \alpha)}^{\min \left\{1, \frac{2}{r}\right\}} .
$$

We substitute this into the right-hand side of (4.9) to eliminate $\left\|\mathbf{u}-\mathbf{u}_{h}\right\|_{\mathrm{V}}$; thus,

$$
\begin{aligned}
\left|\mathbf{u}-\mathbf{u}_{h}\right|_{(\mathbf{u}, r, \alpha)}^{2} \leq & C\left(\left\|\mathbf{S}_{1}\right\|_{\mathrm{V}^{\prime}}\left|\mathbf{u}-\mathbf{u}_{h}\right|_{(\mathbf{u}, r, \alpha)}^{\min \left\{1, \frac{2}{r}\right\}}+\left\|\mathbf{S}_{1}\right\|_{\mathrm{V}^{\prime}}\left\|\mathrm{S}_{2}\right\|_{\mathrm{Q}^{\prime}}\right. \\
& \left.+\left\|\mathrm{S}_{2}\right\|_{\mathrm{Q}^{\prime}}\left|\mathbf{u}-\mathbf{u}_{h}\right|_{(\mathbf{u}, r, \alpha)}^{\min \left\{1, \frac{2}{r}\right\}}\right),
\end{aligned}
$$

where $C=C\left(K_{1}, K_{2}, c_{0}, r,\|\mathbf{f}\|_{\mathrm{V}^{\prime}}\right)$ is a positive constant. We apply Young's inequality

$$
a b \leq \varepsilon^{1-s} \frac{a^{s}}{s}+\varepsilon \frac{b^{s^{\prime}}}{s^{\prime}}, \quad 1 / s+1 / s^{\prime}=1, \quad 1<s<\infty, \quad a, b \geq 0, \quad \varepsilon>0,
$$

to the first and the third term on the right-hand side of (4.12), with $a=C\left\|\mathbf{S}_{1}\right\|_{\mathrm{V}^{\prime}}$ and $s^{\prime}=2 / \min \{1,2 / r\}=\max \{r, 2\}$ in the case of the first term and $a=C\left\|\mathrm{~S}_{2}\right\|_{\mathrm{Q}^{\prime}}$ and $s^{\prime}=2 / \min \left\{1,2 / r^{\prime}\right\}=\max \left\{r^{\prime}, 2\right\}$ in the case of the third term, and take $\varepsilon>0$ sufficiently small so as to hide the term $\varepsilon\left(1 / \max \{r, 2\}+1 / \max \left\{r^{\prime}, 2\right\}\right)\left|\mathbf{u}-\mathbf{u}_{h}\right|_{(\mathbf{u}, r, \alpha)}^{2}$ thus resulting from the right-hand side of (4.12) into $\left|\mathbf{u}-\mathbf{u}_{h}\right|_{(\mathbf{u}, r, \alpha)}^{2}$ appearing on the left-hand side of (4.12); any $\varepsilon \in(0,1)$ will suffice. Hence we deduce that

$$
\left|\mathbf{u}-\mathbf{u}_{h}\right|_{(\mathbf{u}, r, \alpha)}^{2} \leq C\left(\left\|\mathrm{~S}_{1}\right\|_{\mathrm{V}^{\prime}}^{\mathrm{R}_{\mathrm{U}}^{\prime}}+\left\|\mathrm{S}_{1}\right\|_{\mathrm{V}^{\prime}}\left\|\mathrm{S}_{2}\right\|_{\mathrm{Q}^{\prime}}+\left\|\mathrm{S}_{2}\right\|_{\mathrm{Q}^{\prime}}^{\mathrm{G}^{\prime}}\right)
$$

where $\mathrm{R}_{\mathrm{U}}=\max \{r, 2\}, \mathrm{s}=\max \left\{r^{\prime}, 2\right\}, 1 / \mathrm{R}_{\mathrm{U}}+1 / \mathrm{R}_{\mathrm{U}}^{\prime}=1,1 / \mathrm{s}+1 / \mathrm{s}^{\prime}=1$, and $C$ is a constant depending only on $K_{1}, K_{2}, c_{0}, r,\|\mathbf{f}\|_{\mathrm{V}^{\prime}}$.

Proposition 13 Let $(\mathbf{u}, p) \in \mathrm{V} \times \mathrm{Q}$ denote the solution to (1.4), (1.5), and let $\left(\mathbf{u}_{h}, p_{h}\right) \in$ $\mathrm{V}_{h} \times \mathrm{Q}_{h}$ denote its finite element approximation defined by (2.2), (2.3); then, there exists a positive constant $C=C\left(K_{1}, K_{2}, c_{0}, r,\|\mathbf{f}\|_{\mathrm{V}^{\prime}}\right)$ such that

$$
\left\|\mathbf{u}-\mathbf{u}_{h}\right\|_{\mathrm{V}}^{\mathrm{R}_{\mathrm{U}}}+\left\|p-p_{h}\right\|_{\mathrm{Q}}^{\mathrm{g}} \leq C\left(\left\|\mathbf{S}_{1}\right\|_{\mathrm{V}^{\prime}}^{\mathrm{g}}+\left\|\mathbf{S}_{1}\right\|_{\mathrm{V}^{\prime}}^{\mathrm{R}_{\mathrm{U}}^{\prime}}+\left\|\mathrm{S}_{2}\right\|_{\mathrm{Q}^{\prime}}^{\mathrm{g}^{\prime}}+\left\|\mathbf{S}_{1}\right\|_{\mathrm{V}^{\prime}}\left\|\mathrm{S}_{2}\right\|_{\mathrm{Q}^{\prime}}\right),
$$

where $\mathrm{R}_{\mathrm{U}}=\max \{r, 2\}, \mathrm{g}=\max \left\{r^{\prime}, 2\right\}, 1 / \mathrm{R}_{\mathrm{U}}+1 / \mathrm{R}_{\mathrm{U}}^{\prime}=1,1 / \mathrm{g}+1 / \mathrm{g}^{\prime}=1$. 
Proof. Part (iii) of Proposition 7 with $\mathbf{v}=\mathbf{u}-\mathbf{u}_{h}$ and $\mathbf{w}=\mathbf{u}$, (4.11) and (4.13) imply that

$$
\left\|\mathbf{u}-\mathbf{u}_{h}\right\|_{\mathrm{V}}^{\mathrm{R}_{\mathrm{U}}} \leq C\left(\left\|\mathrm{~S}_{1}\right\|_{\mathrm{V}^{\prime}}^{\mathrm{R}_{\mathrm{U}}^{\prime}}+\left\|\mathrm{S}_{1}\right\|_{\mathrm{V}^{\prime}}\left\|\mathrm{S}_{2}\right\|_{\mathrm{Q}^{\prime}}+\left\|\mathrm{S}_{2}\right\|_{\mathrm{Q}^{\prime}}^{\mathrm{g}^{\prime}}\right) .
$$

For the error in the pressure, substitution of (4.13) into the right-hand side of (4.6) yields

$$
\left\|p-p_{h}\right\|_{\mathrm{Q}}^{\mathrm{g}} \leq C\left(\left\|\mathbf{S}_{1}\right\|_{\mathrm{V}^{\prime}}^{\mathrm{g}}+\left\|\mathbf{S}_{1}\right\|_{\mathrm{V}^{\prime}}^{\mathrm{R}_{\mathrm{U}}^{\prime}}+\left\|\mathrm{S}_{2}\right\|_{\mathrm{Q}^{\prime}}^{\mathrm{g}^{\prime}}+\left\|\mathbf{S}_{1}\right\|_{\mathrm{V}^{\prime}}\left\|\mathrm{S}_{2}\right\|_{\mathrm{Q}^{\prime}}\right) .
$$

Inequalities (4.15) and (4.16) simply yield (4.14) and that completes the proof of Proposition 13 .

In the special case of a power-law model, $k(t)=2 \mu t^{r-2}$ with $r \in(1,2)$, the bounds (4.15) and (4.16) collapse to those of Sandri [22].

The a posteriori error bounds stated in Proposition 13 can be simplified, thus leading to Theorem 14.

Theorem 14 Let $(\mathbf{u}, p) \in \mathrm{V} \times \mathrm{Q}$ denote the solution to (1.4), (1.5), and let $\left(\mathbf{u}_{h}, p_{h}\right) \in$ $\mathrm{V}_{h} \times \mathrm{Q}_{h}$ denote its finite element approximation defined by (2.2), (2.3). Then, there exists a positive constant $C$ depending on $K_{1}, K_{2}, c_{0}, c_{0}^{\prime}, r,\|\mathbf{f}\|_{\mathrm{V}^{\prime}}$ such that

$$
\left\|\mathbf{u}-\mathbf{u}_{h}\right\|_{\mathrm{V}}^{\mathrm{R}_{\mathrm{U}}}+\left\|p-p_{h}\right\|_{\mathrm{Q}}^{\mathrm{g}} \leq C\left(\left\|\mathrm{~S}_{1}\right\|_{\mathrm{V}^{\prime}}^{\mathrm{R}^{\prime}}+\left\|\mathrm{S}_{2}\right\|_{\mathrm{Q}^{\prime}}^{\mathrm{g}^{\prime}}\right),
$$

where $\mathrm{R}_{\mathrm{U}}=\max \{r, 2\}, \mathrm{я}=\max \left\{r^{\prime}, 2\right\}, 1 / \mathrm{R}_{\mathrm{U}}+1 / \mathrm{R}_{\mathrm{U}}^{\prime}=1,1 / \mathrm{s}+1 / \mathrm{g}^{\prime}=1$.

Proof. By virtue of (4.1) and Remark 10,

$$
\left\|\mathbf{S}_{1}\right\|_{\mathrm{V}^{\prime}} \leq\left(1+\frac{1}{c_{0}^{\prime}}\right)\left(\|\mathbf{f}\|_{\mathrm{V}^{\prime}}+K_{2}\left(H \circ G^{-1}\right)\left(\frac{1}{K_{1}}\|\mathbf{f}\|_{\mathrm{V}^{\prime}}\right)\right) .
$$

Thus, on noting that $\mathrm{R}_{\mathrm{U}}^{\prime}=\min \left\{r^{\prime}, 2\right\} \leq \max \left\{r^{\prime}, 2\right\}=$ я, we have that

$$
\left\|\mathbf{S}_{1}\right\|_{\mathrm{V}^{\prime}}^{\mathrm{R}_{\mathrm{U}}^{\prime}}+\left\|\mathbf{S}_{1}\right\|_{\mathrm{V}^{\prime}}^{\mathrm{g}}=\left\|\mathbf{S}_{1}\right\|_{\mathrm{V}^{\prime}}^{\mathrm{R}^{\prime}}\left(1+\left\|\mathbf{S}_{1}\right\|_{\mathrm{V}^{\prime}}^{\mathrm{g}-\mathrm{R}_{\mathrm{U}}^{\prime}}\right) \leq C\left\|\mathbf{S}_{1}\right\|_{\mathrm{V}^{\prime}}^{\mathrm{R}_{\mathrm{U}}^{\prime}}
$$

where $C=C\left(K_{1}, K_{2}, c_{0}^{\prime}, r,\|\mathbf{f}\|_{\mathrm{V}^{\prime}}\right)$. Also, by Young's inequality and (4.18),

$$
\left\|\mathrm{S}_{1}\right\|_{\mathrm{V}^{\prime}}\left\|\mathrm{S}_{2}\right\|_{\mathrm{Q}^{\prime}} \leq \frac{1}{\mathrm{~g}}\left\|\mathrm{~S}_{1}\right\|_{\mathrm{V}^{\prime}}^{\mathrm{g}}+\frac{1}{\mathrm{~g}^{\prime}}\left\|\mathrm{S}_{2}\right\|_{\mathrm{Q}^{\prime}}^{\mathrm{g}^{\prime}} \leq C\left(\left\|\mathrm{~S}_{1}\right\|_{\mathrm{V}^{\prime}}^{\mathrm{R}_{\mathrm{U}}^{\prime}}+\left\|\mathrm{S}_{2}\right\|_{\mathrm{Q}^{\prime}}^{\mathrm{g}^{\prime}}\right)
$$

where $C=C\left(K_{1}, K_{2}, c_{0}^{\prime}, r,\|\mathbf{f}\|_{\mathrm{V}^{\prime}}\right)$. Hence, (4.18), (4.19), together with (4.15) and (4.16) of Proposition 13, yield the a posteriori error bound (4.17).

\subsection{Upper bound on the error in terms of computable residuals}

Since $\left\|\mathrm{S}_{1}\right\|_{\mathrm{V}^{\prime}}$ and $\left\|\mathrm{S}_{2}\right\|_{\mathrm{Q}^{\prime}}$ can be bounded in terms of the following residuals:

$$
\begin{aligned}
\mathbf{R}_{T}\left(\left[\mathbf{u}_{h}, p_{h}\right]\right) & =\nabla \cdot\left(k\left(x,\left|e\left(\mathbf{u}_{h}\right)\right|\right) e\left(\mathbf{u}_{h}\right)\right)-\nabla p+\left.\mathbf{f}\right|_{T} & & \forall T \in \mathcal{T}_{h}, \\
\mathbf{J}_{E}\left(\left[\mathbf{u}_{h}, p_{h}\right]\right) & =\llbracket \hat{n}_{E} \cdot\left(k\left(x,\left|e\left(\mathbf{u}_{h}\right)\right|\right) e\left(\mathbf{u}_{h}\right)-p_{h} \mathbf{I}\right) \rrbracket_{E} & & \forall E \in \mathcal{E}_{h, \Omega}
\end{aligned}
$$


and $\nabla \cdot \mathbf{u}_{h}$, we can easily obtain the desired a posteriori upper bound in terms of computable quantities stated in the first part of Theorem 2. Before doing so, we need to introduce two operators, $\Pi_{T}$ and $\Pi_{E}$, which associate to a given function $u$, defined on an element $T$ or on a face $E$, a suitable polynomial approximation on $T$ or $E$, respectively. In particular, we can consider these approximations as being related to numerical quadrature formulae.

Theorem 15 Let $(\mathbf{u}, p) \in \mathrm{V} \times \mathrm{Q}$ denote the solution to (1.4), (1.5), and let $\left(\mathbf{u}_{h}, p_{h}\right) \in$ $\mathrm{V}_{h} \times \mathrm{Q}_{h}$ denote its finite element approximation defined by (2.2), (2.3). Then, there exists a positive constant $C_{U}$ depending on $K_{1}, K_{2}, c_{0}, c_{0}^{\prime}, r,\|\mathbf{f}\|_{\mathrm{V}^{\prime}}$ and on the minimal angle of the triangulation such that

$$
\begin{aligned}
\left\|\mathbf{u}-\mathbf{u}_{h}\right\|_{\mathrm{V}}^{\mathrm{R}_{\mathrm{U}}}+ & \left\|p-p_{h}\right\|_{\mathrm{Q}}^{\text {g }} \leq C_{U}\left[\left(\sum_{T \in \mathcal{T}_{h}} h_{T}^{r^{\prime}}\left\|\Pi_{T} \mathbf{R}_{T}\left(\left[\mathbf{u}_{h}, p_{h}\right]\right)\right\|_{L^{r^{\prime}}(T)}^{r^{\prime}}\right)^{\frac{\mathrm{R}_{\mathrm{U}}^{\prime}}{r^{\prime}}}\right. \\
+ & \left(\sum_{E \in \mathcal{E}_{h, \Omega}} h_{E}\left\|\Pi_{E} \mathbf{J}_{E}\left(\left[\mathbf{u}_{h}, p_{h}\right]\right)\right\|_{L^{r^{\prime}}(E)}^{r^{\prime}}\right)^{\frac{\mathrm{R}_{U}^{\prime}}{r^{\prime}}}+\left\|\nabla \cdot \mathbf{u}_{h}\right\|_{L^{r^{\prime}}(\Omega)}^{\mathrm{g}^{\prime}} \\
& +\left(\sum_{T \in \mathcal{T}_{h}} h_{T}^{r^{\prime}}\left\|\mathbf{R}_{T}\left(\left[\mathbf{u}_{h}, p_{h}\right]\right)-\Pi_{T} \mathbf{R}_{T}\left(\left[\mathbf{u}_{h}, p_{h}\right]\right)\right\|_{L^{r^{\prime}}(T)}^{r^{\prime}}\right)^{\frac{\mathrm{R}_{\mathrm{U}}^{\prime}}{r^{\prime}}} \\
+ & \left.\left(\sum_{E \in \mathcal{E}_{h, \Omega}} h_{E}\left\|\mathbf{J}_{E}\left(\left[\mathbf{u}_{h}, p_{h}\right]\right)-\Pi_{E} \mathbf{J}_{E}\left(\left[\mathbf{u}_{h}, p_{h}\right]\right)\right\|_{L^{r^{\prime}}(E)}^{r^{\prime}}\right)^{\frac{\mathrm{R}_{\mathrm{U}}^{\prime}}{r^{\prime}}}\right]
\end{aligned}
$$

where $\mathrm{R}_{\mathrm{U}}=\max \{r, 2\}, \mathrm{g}=\max \left\{r^{\prime}, 2\right\}, 1 / \mathrm{R}_{\mathrm{U}}+1 / \mathrm{R}_{\mathrm{U}}^{\prime}=1,1 / \mathrm{s}+1 / \mathrm{s}^{\prime}=1$.

Proof. We deduce from (1.4) and (1.5) that, for all $\mathbf{w} \in \mathrm{V}$ and all $q \in \mathrm{Q}$,

$$
\begin{aligned}
a(\mathbf{u}, \mathbf{w})-a\left(\mathbf{u}_{h}, \mathbf{w}\right)+b\left(p-p_{h}, \mathbf{w}\right) & =(\mathbf{f}, \mathbf{w})-a\left(\mathbf{u}_{h}, \mathbf{w}\right)-b\left(p_{h}, \mathbf{w}\right) \\
b\left(q, \mathbf{u}-\mathbf{u}_{h}\right) & =-b\left(q, \mathbf{u}_{h}\right) .
\end{aligned}
$$

Adding (4.21) to (4.22) and using (2.2) with $\mathbf{v}_{h}=I_{h} \mathbf{w}$ yields

$$
\begin{aligned}
& a(\mathbf{u}, \mathbf{w})-a\left(\mathbf{u}_{h}, \mathbf{w}\right)+b\left(p-p_{h}, \mathbf{w}\right)+b\left(q, \mathbf{u}-\mathbf{u}_{h}\right) \\
& \quad=\left\{\left(\mathbf{f}, \mathbf{w}-I_{h} \mathbf{w}\right)-a\left(\mathbf{u}_{h}, \mathbf{w}-I_{h} \mathbf{w}\right)-b\left(p_{h}, \mathbf{w}-I_{h} \mathbf{w}\right)\right\}-b\left(q, \mathbf{u}_{h}\right)
\end{aligned}
$$

for all $\mathbf{w} \in \mathrm{V}$ and all $q \in \mathrm{Q}$.

We proceed by decomposing the inner product $(\cdot, \cdot)$, the semilinear form $a(\cdot, \cdot)$ and the bilinear form $b(\cdot, \cdot)$ as sums of integrals over elements $T \in \mathcal{T}_{h}$, and integrating by 
parts over each element $T \in \mathcal{T}_{h}$; thus,

$$
\begin{aligned}
& a(\mathbf{u}, \mathbf{w})-a\left(\mathbf{u}_{h}, \mathbf{w}\right)+b\left(p-p_{h}, \mathbf{w}\right)+b\left(q, \mathbf{u}-\mathbf{u}_{h}\right)=\sum_{T \in \mathcal{T}_{h}} \int_{T} \mathbf{f} \cdot\left(\mathbf{w}-I_{h} \mathbf{w}\right) \mathrm{d} \Omega \\
& -\sum_{T \in \mathcal{T}_{h}} \int_{T} k\left(\left|e\left(\mathbf{u}_{h}\right)\right|\right) e\left(\mathbf{u}_{h}\right): e\left(\mathbf{w}-I_{h} \mathbf{w}\right) \mathrm{d} \Omega \\
& +\sum_{T \in \mathcal{T}_{h}} \int_{T} p_{h} \nabla \cdot\left(\mathbf{w}-I_{h} \mathbf{w}\right) \mathrm{d} \Omega+\sum_{T \in \mathcal{T}_{h}} \int_{T}\left(\nabla \cdot \mathbf{u}_{h}\right) q \mathrm{~d} \Omega \\
& =\sum_{T \in \mathcal{T}_{h}} \int_{T}\left(\mathbf{f}+\nabla \cdot\left(k\left(\left|e\left(\mathbf{u}_{h}\right)\right|\right) e\left(\mathbf{u}_{h}\right)\right)-\nabla p_{h}\right) \cdot\left(\mathbf{w}-I_{h} \mathbf{w}\right) \mathrm{d} \Omega \\
& -\sum_{T \in \mathcal{T}_{h}} \int_{\partial T}\left[k\left(\left|e\left(\mathbf{u}_{h}\right)\right|\right) e\left(\mathbf{u}_{h}\right) \mathbf{n}_{T}-p_{h} \mathbf{n}_{T}\right] \cdot\left(\mathbf{w}-I_{h} \mathbf{w}\right) \mathrm{d} \Gamma \\
& +\sum_{T \in \mathcal{T}_{h}} \int_{T}\left(\nabla \cdot \mathbf{u}_{h}\right) q \mathrm{~d} \Omega \\
& =\sum_{T \in \mathcal{T}_{h}} \int_{T} \mathbf{R}_{T}\left(\left[\mathbf{u}_{h}, p_{h}\right]\right) \cdot\left(\mathbf{w}-I_{h} \mathbf{w}\right) \mathrm{d} \Omega \\
& -\sum_{E \in \mathcal{E}_{h, \Omega}} \int_{E} \mathbf{J}_{E}\left(\left[\mathbf{u}_{h}, p_{h}\right]\right) \cdot\left(\mathbf{w}-I_{h} \mathbf{w}\right) \mathrm{d} \Gamma \\
& +\sum_{T \in \mathcal{T}_{h}} \int_{T}\left(\nabla \cdot \mathbf{u}_{h}\right) q \mathrm{~d} \Omega, \\
& =\sum_{T \in \mathcal{T}_{h}} \int_{T} \Pi_{T} \mathbf{R}_{T}\left(\left[\mathbf{u}_{h}, p_{h}\right]\right) \cdot\left(\mathbf{w}-I_{h} \mathbf{w}\right) \mathrm{d} \Omega \\
& +\sum_{T \in \mathcal{T}_{h}} \int_{T}\left(\mathbf{R}_{T}\left(\left[\mathbf{u}_{h}, p_{h}\right]\right)-\Pi_{T} \mathbf{R}_{T}\left(\left[\mathbf{u}_{h}, p_{h}\right]\right)\right) \cdot\left(\mathbf{w}-I_{h} \mathbf{w}\right) \mathrm{d} \Omega \\
& -\sum_{E \in \mathcal{E}_{h, \Omega}} \int_{E} \Pi_{E} \mathbf{J}_{E}\left(\left[\mathbf{u}_{h}, p_{h}\right]\right) \cdot\left(\mathbf{w}-I_{h} \mathbf{w}\right) \mathrm{d} \Gamma \\
& -\sum_{E \in \mathcal{E}_{h, \Omega}} \int_{E}\left(\mathbf{J}_{E}\left(\left[\mathbf{u}_{h}, p_{h}\right]\right)-\Pi_{E} \mathbf{J}_{E}\left(\left[\mathbf{u}_{h}, p_{h}\right]\right)\right) \cdot\left(\mathbf{w}-I_{h} \mathbf{w}\right) \mathrm{d} \Gamma \\
& +\sum_{T \in \mathcal{T}_{h}} \int_{T}\left(\nabla \cdot \mathbf{u}_{h}\right) q \mathrm{~d} \Omega
\end{aligned}
$$

where $\mathbf{n}_{T}$ is the unit outward normal vector to the boundary $\partial T$ of the simplex $T \in \mathcal{T}_{h}$. As $\mathbf{w}-I_{h} \mathbf{w}=\mathbf{0}$ on $\partial \Omega$, parts of $\partial T$ which intersect $\partial \Omega$ can be omitted from the region of integration. Hence, only faces $E \subset \partial T$ internal to $\Omega$ need to be considered in detail.

Let $E \subset \Omega$ be a $(d-1)$-dimensional face shared by elements $T_{\sharp}$ and $T_{b}$. Then, during the summation $\sum_{T \in \mathcal{T}_{h}}$ and surface integration $\int_{\partial T} \ldots \mathrm{d} \Gamma$, the face $E$ will be traversed twice: once in the course of integration over $\partial T_{\sharp}$ and then in the course of integration 


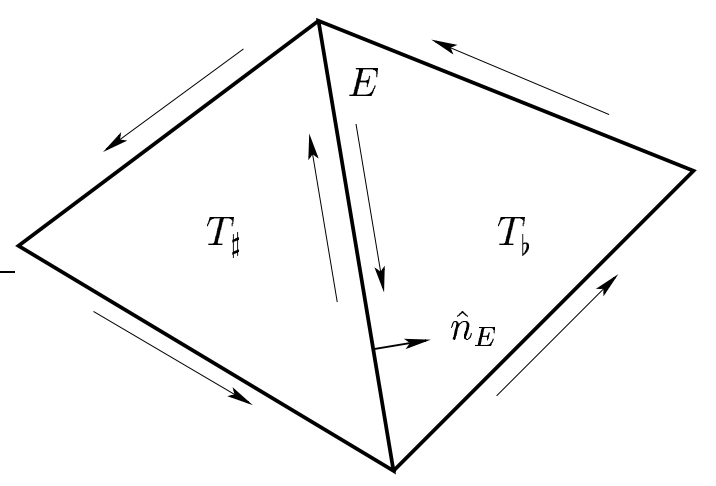

Figure 3: A face $E$ shared by elements $T_{\sharp}$ and $T_{b}$ in the triangulation and the unit outward normal vector $\hat{n}_{T_{\sharp}} \equiv \hat{n}_{E}$ to the boundary $\partial T_{\sharp}$ of $T_{\sharp}$, in the case $d=2$

over $\partial T_{b}$ (cf. Figure 3).

Since during the two passes through $E=\partial T_{\sharp} \cap \partial T_{b}$ the orientation of the unit outward normal changes, we get the jump

$$
\llbracket \mathbf{v} \rrbracket_{E}=\left.\mathbf{v} \cdot \hat{n}_{T_{\sharp}}\right|_{\partial T_{\sharp} \cap \Omega}+\left.\mathbf{v} \cdot \hat{n}_{T_{\mathrm{b}}}\right|_{\partial T_{\mathrm{b}} \cap \Omega}=\left.\mathbf{v} \cdot \hat{n}_{E}\right|_{\partial T_{\sharp} \cap \Omega}-\left.\mathbf{v} \cdot \hat{n}_{E}\right|_{\partial T_{\mathrm{b}} \cap \Omega} .
$$

If we write the sum $\sum_{E \in \mathcal{E}_{h, \Omega}}$ of the jumps $\Pi_{E} \mathbf{J}_{E}\left(\left[\mathbf{u}_{h}, p_{h}\right]\right)$ as $\sum_{T \in \mathcal{T}_{h}} \sum_{E \in \mathcal{E}(T) \cap \Omega}$ we need to introduce a factor $\frac{1}{2}$, due to the fact that in the double summation over $T \in \mathcal{T}_{h}$ and $E \in \mathcal{E}(T) \cap \Omega$ each face $E$ has been counted twice:

$$
\sum_{E \in \mathcal{E}_{h}, \Omega} \int_{E} \Pi_{E} \mathbf{J}_{E}\left(\left[\mathbf{u}_{h}, p_{h}\right]\right) \cdot\left(\mathbf{w}-I_{h} \mathbf{w}\right) \mathrm{d} \Gamma=\frac{1}{2} \sum_{T \in \mathcal{T}_{h}} \sum_{E \in \mathcal{E}(T) \cap \Omega} \int_{E} \Pi_{E} \mathbf{J}_{E}\left(\left[\mathbf{u}_{h}, p_{h}\right]\right) \cdot\left(\mathbf{w}-I_{h} \mathbf{w}\right) \mathrm{d} \Gamma .
$$

On expressing the right-hand side of (4.23) in terms of $\Pi_{T} \mathbf{R}_{T}\left(\left[\mathbf{u}_{h}, p_{h}\right]\right), \Pi_{E} \mathbf{J}_{E}\left(\left[\mathbf{u}_{h}, p_{h}\right]\right)$ and $\nabla \cdot \mathbf{u}_{h}$, we obtain the following error representation formula:

$$
\begin{aligned}
& a(\mathbf{u}, \mathbf{w})-a\left(\mathbf{u}_{h}, \mathbf{w}\right)+b\left(p-p_{h}, \mathbf{w}\right)+b\left(q, \mathbf{u}-\mathbf{u}_{h}\right) \\
& =\sum_{T \in \mathcal{T}_{h}} \int_{T} \Pi_{T} \mathbf{R}_{T}\left(\left[\mathbf{u}_{h}, p_{h}\right]\right) \cdot\left(\mathbf{w}-I_{h} \mathbf{w}\right) \mathrm{d} \Omega \\
& \quad+\sum_{T \in \mathcal{T}_{h}} \int_{T}\left(\mathbf{R}_{T}\left(\left[\mathbf{u}_{h}, p_{h}\right]\right)-\Pi_{T} \mathbf{R}_{T}\left(\left[\mathbf{u}_{h}, p_{h}\right]\right)\right) \cdot\left(\mathbf{w}-I_{h} \mathbf{w}\right) \mathrm{d} \Omega \\
& \quad-\sum_{E \in \mathcal{E}_{h, \Omega}} \int_{E} \Pi_{E} \mathbf{J}_{E}\left(\left[\mathbf{u}_{h}, p_{h}\right]\right) \cdot\left(\mathbf{w}-I_{h} \mathbf{w}\right) \mathrm{d} \Gamma \\
& \quad-\sum_{E \in \mathcal{E}_{h, \Omega}} \int_{E}\left(\mathbf{J}_{E}\left(\left[\mathbf{u}_{h}, p_{h}\right]\right)-\Pi_{E} \mathbf{J}_{E}\left(\left[\mathbf{u}_{h}, p_{h}\right]\right)\right) \cdot\left(\mathbf{w}-I_{h} \mathbf{w}\right) \mathrm{d} \Gamma \\
& \quad+\sum_{T \in \mathcal{T}_{h}} \int_{T} \nabla \cdot \mathbf{u}_{h}(q+c) \mathrm{d} \Omega
\end{aligned}
$$


for all $\mathbf{w} \in \mathrm{V}$, all $q \in \mathrm{Q}$, and all $c \in \mathbb{R}$; here we made use of the fact that

$$
c \sum_{T \in \mathcal{T}_{h}} \int_{T} \nabla \cdot \mathbf{u}_{h} \mathrm{~d} \Omega=c \int_{\Omega} \nabla \cdot \mathbf{u}_{h} \mathrm{~d} \Omega=c \int_{\partial \Omega} \mathbf{u}_{h} \cdot \mathbf{n} \mathrm{d} \Gamma=0
$$

for all $c \in \mathbb{R}$ since $\left.\mathbf{u}_{h}\right|_{\partial \Omega}=\mathbf{0}$.

Applying Hölder's inequality to each of the terms on the right-hand side of (4.25) and then taking the infimum over all $c \in \mathbb{R}$, we have that

$$
\begin{aligned}
a(\mathbf{u}, \mathbf{w})- & a\left(\mathbf{u}_{h}, \mathbf{w}\right)+b\left(p-p_{h}, \mathbf{w}\right)+b\left(q, \mathbf{u}-\mathbf{u}_{h}\right) \\
\leq & \sum_{T \in \mathcal{T}_{h}}\left\|\Pi_{T} \mathbf{R}_{T}\left(\left[\mathbf{u}_{h}, p_{h}\right]\right)\right\|_{L^{r^{\prime}}(T)}\left\|\mathbf{w}-I_{h} \mathbf{w}\right\|_{L^{r}(T)} \\
& +\sum_{T \in \mathcal{T}_{h}}\left\|\mathbf{R}_{T}\left(\left[\mathbf{u}_{h}, p_{h}\right]\right)-\Pi_{T} \mathbf{R}_{T}\left(\left[\mathbf{u}_{h}, p_{h}\right]\right)\right\|_{L^{r^{\prime}(T)}}\left\|\mathbf{w}-I_{h} \mathbf{w}\right\|_{L^{r}(T)} \\
& +\sum_{E \in \mathcal{E}_{h, \Omega}}\left\|\Pi_{E} \mathbf{J}_{E}\left(\left[\mathbf{u}_{h}, p_{h}\right]\right)\right\|_{L^{r^{\prime}(E)}}\left\|\mathbf{w}-I_{h} \mathbf{w}\right\|_{L^{r}(E)} \\
& +\sum_{E \in \mathcal{E}_{h, \Omega}}\left\|\mathbf{J}_{E}\left(\left[\mathbf{u}_{h}, p_{h}\right]\right)-\Pi_{E} \mathbf{J}_{E}\left(\left[\mathbf{u}_{h}, p_{h}\right]\right)\right\|_{\left.L^{r^{\prime}(E)}\right)}\left\|\mathbf{w}-I_{h} \mathbf{w}\right\|_{L^{r}(E)} \\
& +\sum_{T \in \mathcal{T}_{h}}\left\|\nabla \cdot \mathbf{u}_{h}\right\|_{L^{r}(T)}{\underset{i n f}{c \in \mathbb{R}}}^{\inf }\|q\|_{L^{r^{\prime}}(T)}
\end{aligned}
$$

for all $\mathbf{w} \in \mathrm{V}$ and all $q \in \mathrm{Q}$.

Applying (2.4) and (2.5) in (4.26) and using Hölder's inequality for finite sums, we have that

$$
\begin{aligned}
a(\mathbf{u}, \mathbf{w})- & a\left(\mathbf{u}_{h}, \mathbf{w}\right)+b\left(p-p_{h}, \mathbf{w}\right)+b\left(q, \mathbf{u}-\mathbf{u}_{h}\right) \\
\leq & C\left(\sum_{T \in \mathcal{T}_{h}} h_{T}^{r^{\prime}}\left\|\Pi_{T} \mathbf{R}_{T}\left(\left[\mathbf{u}_{h}, p_{h}\right]\right)\right\|_{L^{r^{\prime}(T)}}^{r^{\prime}}\right)^{1 / r^{\prime}}|\mathbf{w}|_{\mathrm{W}^{1, r}(\Omega)} \\
& +C\left(\sum_{T \in \mathcal{T}_{h}} h_{T}^{r^{\prime}}\left\|\mathbf{R}_{T}\left(\left[\mathbf{u}_{h}, p_{h}\right]\right)-\Pi_{T} \mathbf{R}_{T}\left(\left[\mathbf{u}_{h}, p_{h}\right]\right)\right\|_{L^{r^{\prime}(T)}}^{r^{\prime}}\right)^{1 / r^{\prime}}|\mathbf{w}|_{\mathrm{W}^{1, r}(\Omega)} \\
& +C\left(\sum_{E \in \mathcal{E}_{h, \Omega}} h_{T}\left\|\Pi_{E} \mathbf{J}_{E}\left(\left[\mathbf{u}_{h}, p_{h}\right]\right)\right\|_{L^{r^{\prime}(E)}}^{r^{\prime}}\right)^{1 / r^{\prime}}|\mathbf{w}|_{\mathrm{W}^{1, r}(\Omega)}|\mathbf{w}|_{\mathrm{W}^{1, r}(\Omega)} \\
& +C\left(\sum_{E \in \mathcal{E}_{h, \Omega}} h_{T}\left\|\mathbf{J}_{E}\left(\left[\mathbf{u}_{h}, p_{h}\right]\right)-\Pi_{E} \mathbf{J}_{E}\left(\left[\mathbf{u}_{h}, p_{h}\right]\right)\right\|_{L^{r^{\prime}}(E)}^{r^{\prime}}\right)^{1 / r^{\prime}} \\
& +\left(\sum_{T \in \mathcal{T}_{h}}\left\|\nabla \cdot \mathbf{u}_{h}\right\|_{L^{r}(T)}^{r}\right)^{1 / r} \inf _{c \in \mathbb{R}}\|q+c\|_{L^{r^{\prime}}(\Omega)}
\end{aligned}
$$


for all $\mathbf{w} \in \mathrm{V}$ and all $q \in \mathrm{Q}$. Inequality (4.27) implies that

$$
\begin{aligned}
a(\mathbf{u}, \mathbf{w})- & a\left(\mathbf{u}_{h}, \mathbf{w}\right)+b\left(p-p_{h}, \mathbf{w}\right)+b\left(q, \mathbf{u}-\mathbf{u}_{h}\right) \\
\leq C & {\left[\left(\sum_{T \in \mathcal{T}_{h}} h_{T}^{r^{\prime}}\left\|\Pi_{T} \mathbf{R}_{T}\left(\left[\mathbf{u}_{h}, p_{h}\right]\right)\right\|_{L^{r^{\prime}}(T)}^{r^{\prime}}\right)^{\frac{1}{r^{\prime}}}\right.} \\
& +\left(\sum_{T \in \mathcal{T}_{h}} h_{T}^{r^{\prime}}\left\|\mathbf{R}_{T}\left(\left[\mathbf{u}_{h}, p_{h}\right]\right)-\Pi_{T} \mathbf{R}_{T}\left(\left[\mathbf{u}_{h}, p_{h}\right]\right)\right\|_{L^{r^{\prime}}(T)}^{r^{\prime}}\right)^{\frac{1}{r^{\prime}}} \\
& +\left(\sum_{E \in \mathcal{E}_{h, \Omega}} h_{T}\left\|\Pi_{E} \mathbf{J}_{E}\left(\left[\mathbf{u}_{h}, p_{h}\right]\right)\right\|_{L^{r^{\prime}}(E)}^{r^{\prime}}\right)^{\frac{1}{r^{\prime}}} \\
& \left.+\left(\sum_{E \in \mathcal{E}_{h, \Omega}} h_{T}\left\|\mathbf{J}_{E}\left(\left[\mathbf{u}_{h}, p_{h}\right]\right)-\Pi_{E} \mathbf{J}_{E}\left(\left[\mathbf{u}_{h}, p_{h}\right]\right)\right\|_{L^{r^{\prime}}(E)}^{r^{\prime}}\right)^{\frac{1}{r^{\prime}}}\right] \\
& +\left(\sum_{T \in \mathcal{T}_{h}}\left\|\nabla \cdot \mathbf{w}_{h}\right\|_{\mathrm{W}^{1, r}(\Omega)}^{r}\right)_{L^{r}(T)}^{\frac{1}{r^{\prime}}} \inf _{c \in \mathbb{R}}\|q+c\|_{L^{r^{\prime}}(\Omega)}
\end{aligned}
$$

for all $\mathbf{w} \in \mathrm{V}$ and all $q \in \mathrm{Q}$. Taking $q=0$ in (4.28) and then the supremum over all $\mathbf{w} \in \mathrm{V}=\left[\mathrm{W}_{0}^{1, r}(\Omega)\right]^{d}$, using (1.7), and recalling that $\|e(\mathbf{w})\|_{\mathrm{L}^{r}(\Omega)}=\|\mathbf{w}\|_{\mathrm{V}}$, we get

$$
\begin{aligned}
& \sup _{\mathbf{w} \in \mathrm{V}} \frac{a(\mathbf{u}, \mathbf{w})-a\left(\mathbf{u}_{h}, \mathbf{w}\right)+b\left(p-p_{h}, \mathbf{w}\right)}{\|\mathbf{w}\|_{\mathrm{V}}} \\
& \leq C\left[\left(\sum_{T \in \mathcal{T}_{h}} h_{T}^{r^{\prime}}\left\|\Pi_{T} \mathbf{R}_{T}\left(\left[\mathbf{u}_{h}, p_{h}\right]\right)\right\|_{L^{r^{\prime}}(T)}^{r^{\prime}}\right)^{\frac{1}{r^{\prime}}}+\left(\sum_{E \in \mathcal{E}_{h, \Omega}} h_{T}\left\|\Pi_{E} \mathbf{J}_{E}\left(\left[\mathbf{u}_{h}, p_{h}\right]\right)\right\|_{L^{r^{\prime}}(E)}^{r^{\prime}}\right)^{\frac{1}{r^{\prime}}}\right. \\
&+\left(\sum_{T \in \mathcal{T}_{h}} h_{T}^{r^{\prime}}\left\|\mathbf{R}_{T}\left(\left[\mathbf{u}_{h}, p_{h}\right]\right)-\Pi_{T} \mathbf{R}_{T}\left(\left[\mathbf{u}_{h}, p_{h}\right]\right)\right\|_{L^{r^{\prime}}(T)}^{r^{\prime}}\right)^{\frac{1}{r^{\prime}}} \\
&\left.+\left(\sum_{E \in \mathcal{E}_{h, \Omega}} h_{T}\left\|\mathbf{J}_{E}\left(\left[\mathbf{u}_{h}, p_{h}\right]\right)-\Pi_{E} \mathbf{J}_{E}\left(\left[\mathbf{u}_{h}, p_{h}\right]\right)\right\|_{L^{r^{\prime}}(E)}^{r^{\prime}}\right)^{\frac{1}{r^{\prime}}}\right]
\end{aligned}
$$

On the other hand, taking $\mathbf{w}=\mathbf{0}$ in (4.28) and then the supremum over $q \in \mathrm{Q}$ yields

$$
\sup _{q \in \mathrm{Q}} \frac{b\left(q, \mathbf{u}-\mathbf{u}_{h}\right)}{\|q\|_{\mathrm{Q}}} \leq\left(\sum_{T \in \mathcal{T}_{h}}\left\|\nabla \cdot \mathbf{u}_{h}\right\|_{L^{r}(T)}^{r}\right)^{1 / r}
$$

Let us bound the norms $\left\|\mathrm{S}_{1}\right\|_{\mathrm{V}^{\prime}}$ and $\left\|\mathrm{S}_{2}\right\|_{\mathrm{Q}^{\prime}}$. We begin by noting that (1.4) and (4.1) imply that

$$
\left\langle\mathbf{S}_{1}, \mathbf{w}\right\rangle=a(\mathbf{u}, \mathbf{w})-a\left(\mathbf{u}_{h}, \mathbf{w}\right)+b(p, \mathbf{w})-b\left(p_{h}, \mathbf{w}_{h}\right) .
$$


Hence,

$$
\left\|\mathbf{S}_{1}\right\|_{\mathrm{V}^{\prime}}=\sup _{\mathbf{w} \in \mathrm{V}} \frac{\left\langle\mathbf{S}_{1}, \mathbf{w}\right\rangle}{\|\mathbf{w}\|_{\mathrm{V}}}=\sup _{\mathbf{w} \in \mathrm{V}} \frac{a(\mathbf{u}, \mathbf{w})-a\left(\mathbf{u}_{h}, \mathbf{w}\right)+b\left(p-p_{h}, \mathbf{w}\right)}{\|\mathbf{w}\|_{\mathrm{V}}} .
$$

Applying (4.29) to the right-most expression in this chain, we deduce that

$$
\begin{aligned}
\left\|\mathbf{S}_{1}\right\|_{\mathrm{V}^{\prime}} \leq C & {\left[\left(\sum_{T \in \mathcal{T}_{h}} h_{T}^{r^{\prime}}\left\|\Pi_{T} \mathbf{R}_{T}\left(\left[\mathbf{u}_{h}, p_{h}\right]\right)\right\|_{L^{r^{\prime}}(T)}^{r^{\prime}}\right)^{\frac{1}{r^{\prime}}}+\left(\sum_{E \in \mathcal{E}_{h, \Omega}} h_{T}\left\|\Pi_{E} \mathbf{J}_{E}\left(\left[\mathbf{u}_{h}, p_{h}\right]\right)\right\|_{L^{r^{\prime}}(E)}^{r^{\prime}}\right)^{\frac{1}{r^{\prime}}}\right.} \\
& +\left(\sum_{T \in \mathcal{T}_{h}} h_{T}^{r^{\prime}}\left\|\mathbf{R}_{T}\left(\left[\mathbf{u}_{h}, p_{h}\right]\right)-\Pi_{T} \mathbf{R}_{T}\left(\left[\mathbf{u}_{h}, p_{h}\right]\right)\right\|_{L^{r^{\prime}}(T)}^{r^{\prime}}\right)^{\frac{1}{r^{\prime}}} \\
& \left.+\left(\sum_{E \in \mathcal{E}_{h, \Omega}} h_{T}\left\|\mathbf{J}_{E}\left(\left[\mathbf{u}_{h}, p_{h}\right]\right)-\Pi_{E} \mathbf{J}_{E}\left(\left[\mathbf{u}_{h}, p_{h}\right]\right)\right\|_{L^{r^{\prime}}(E)}^{r^{\prime}}\right)^{\frac{1}{r^{\prime}}}\right]
\end{aligned}
$$

Analogously, by (4.2),

$$
\left\|\mathrm{S}_{2}\right\|_{\mathrm{Q}^{\prime}}=\sup _{q \in \mathrm{Q}} \frac{\left\langle\mathrm{S}_{2}, q\right\rangle}{\|q\|_{\mathrm{Q}}}=\sup _{q \in \mathrm{Q}} \frac{-b\left(q, \mathbf{u}_{h}\right)}{\|q\|_{\mathrm{Q}}}=\sup _{q \in \mathrm{Q}} \frac{b\left(q, \mathbf{u}-\mathbf{u}_{h}\right)}{\|q\|_{\mathrm{Q}}}
$$

and therefore, by (4.30),

$$
\left\|\mathrm{S}_{2}\right\|_{\mathrm{Q}^{\prime}} \leq\left(\sum_{T \in \mathcal{T}_{h}}\left\|\nabla \cdot \mathbf{u}_{h}\right\|_{\mathrm{L}^{r}(T)}^{r}\right)^{1 / r}
$$

Combining inequalities (4.31), (4.32) and (4.17) we get the stated bound.

\section{The a posteriori lower bound on the error}

In this section we prove the lower bounds stated in Theorems 1 and 2 .

\subsection{Lower bound on the error in terms of residual functionals}

The following inequalities can be easily obtained by recalling the definitions of $\|\mathbf{u}\|_{\mathrm{W}^{1, r}(\omega)}$, $\|\nabla \cdot \mathbf{u}\|_{L^{r}(\omega)}$ and $\|e(\mathbf{u})\|_{L^{r}(\omega)}$ :

$$
\begin{aligned}
\|\nabla \cdot \mathbf{u}\|_{L^{r}(\omega)} & \leq\|\mathbf{u}\|_{\mathrm{W}^{1, r}(\omega)} \\
\|\nabla \cdot \mathbf{u}\|_{L^{r}(\omega)} & \leq\|e(\mathbf{u})\|_{\mathrm{L}^{r}(\omega)} \\
\|e(\mathbf{u})\|_{\mathrm{L}^{r}(\omega)} & \leq C(r, d)\|\mathbf{u}\|_{\mathrm{W}^{1, r}(\omega)}
\end{aligned}
$$


Theorem 16 Let $(\mathbf{u}, p) \in \mathrm{V} \times \mathrm{Q}$ denote the solution to (1.4), (1.5), and let $\left(\mathbf{u}_{h}, p_{h}\right) \in$ $\mathrm{V}_{h} \times \mathrm{Q}_{h}$ denote its finite element approximation defined by (2.2), (2.3). Then, there exists a positive constant $c$ depending on $K_{1}, K_{2}, r,\|\mathbf{f}\|_{\mathrm{V}^{\prime}}$ such that

$$
c\left[\left\|\mathrm{~S}_{1}\right\|_{\mathrm{V}^{\prime}}^{\mathrm{R}_{\mathrm{L}}^{\prime}}+\left\|\mathrm{S}_{2}\right\|_{\mathrm{Q}^{\prime}}^{\mathrm{G}^{\prime}}\right] \leq\left\|\mathbf{u}-\mathbf{u}_{h}\right\|_{\mathrm{V}}^{\mathrm{R}_{\mathrm{L}}}+\left\|p-p_{h}\right\|_{\mathrm{Q}}^{\mathrm{g}}
$$

where $\mathrm{R}_{\mathrm{L}}=\min \{r, 2\}$, $\mathrm{s}=\max \left\{r^{\prime}, 2\right\}, 1 / \mathrm{R}_{\mathrm{L}}+1 / \mathrm{R}_{\mathrm{L}}^{\prime}=1,1 / \mathrm{s}+1 / \mathrm{g}^{\prime}=1$, and $\mathrm{S}_{1}$ and $\mathrm{S}_{2}$ are the residual functionals defined by (4.1) and (4.2) which are computably bounded.

Proof. Starting from the definition of $\left\|\mathbf{S}_{1}\right\|_{\mathrm{V}^{\prime}}$ we get

$$
\left\|\mathbf{S}_{1}\right\|_{\mathrm{V}^{\prime}}=\sup _{\mathbf{w} \in \mathrm{V}} \frac{\left\langle\mathbf{S}_{1}, \mathbf{w}\right\rangle}{\|\mathbf{w}\|_{\mathrm{V}}} \leq \sup _{\mathbf{w} \in \mathrm{V}} \frac{a(\mathbf{u}, \mathbf{w})-a\left(\mathbf{u}_{h}, \mathbf{w}\right)}{\|\mathbf{w}\|_{\mathrm{V}}}+\sup _{\mathbf{w} \in \mathrm{V}} \frac{b\left(p-p_{h}, \mathbf{w}\right)}{\|\mathbf{w}\|_{\mathrm{V}}} .
$$

The second term on the right-hand side in (5.5) can be bounded, by applying (5.2), in the following way:

$$
\sup _{\mathbf{w} \in \mathrm{V}} \frac{b\left(p-p_{h}, \mathbf{w}\right)}{\|\mathbf{w}\|_{\mathrm{V}}} \leq \sup _{\mathbf{w} \in \mathrm{V}} \frac{\left\|p-p_{h}\right\|_{\mathrm{Q}}\|\nabla \cdot \mathbf{w}\|_{L^{r}(\Omega)}}{\|\mathbf{w}\|_{\mathrm{V}}} \leq\left\|p-p_{h}\right\|_{\mathrm{Q}} .
$$

Next, we use (3.12) in (5.5) to get

$$
\left\|\mathbf{S}_{1}\right\|_{\mathrm{V}^{\prime}} \leq C_{4}\left|\mathbf{u}-\mathbf{u}_{h}\right|_{(\mathbf{u}, r, \alpha)}^{\min \left\{1, \frac{2}{r^{\prime}}\right\}}+\left\|p-p_{h}\right\|_{\mathrm{Q}}
$$

Now let us consider separately the two cases $1<r<2$ and $r \geq 2$.

First suppose that $1<r<2$. Using (iii) of Proposition 7 we have

$$
\left|\mathbf{u}-\mathbf{u}_{h}\right|_{(\mathbf{u}, r, \alpha)}^{\frac{2}{r}} \leq\left\|e\left(\mathbf{u}-\mathbf{u}_{h}\right)\right\|_{L^{r}(\Omega)}
$$

and we conclude that

$$
\begin{array}{r}
\left\|\mathbf{S}_{1}\right\|_{\mathrm{V}^{\prime}} \leq C_{4}\left|\mathbf{u}-\mathbf{u}_{h}\right|_{(\mathbf{u}, r, \alpha)}^{\frac{2}{r^{r}}}+\left\|p-p_{h}\right\|_{\mathrm{Q}} \\
\leq C_{4}\left\|e\left(\mathbf{u}-\mathbf{u}_{h}\right)\right\|_{L^{r}(\Omega)}^{\frac{r}{r^{r}}}+\left\|p-p_{h}\right\|_{\mathrm{Q}} .
\end{array}
$$

On recalling the definition

$$
C_{4}=C_{3} 2^{\max \left\{0, \frac{r-2}{2}\right\}}\left[\Xi_{\alpha} \circ G^{-1}\left(\frac{1}{K_{1}}\|f\|_{\mathrm{V}^{\prime}}\right)\right]^{\max \left\{0, \frac{r-2}{2}\right\}}
$$

from Lemma 11 we get

$$
\left\|\mathbf{S}_{1}\right\|_{\mathrm{V}^{\prime}} \leq C_{3}\left\|e\left(\mathbf{u}-\mathbf{u}_{h}\right)\right\|_{L^{r}(\Omega)}^{\frac{r}{r^{r}}}+\left\|p-p_{h}\right\|_{\mathrm{Q}} .
$$

Now suppose that $r \geq 2$. Again, using (iii) of Proposition 7 we have

$$
\left|\mathbf{u}-\mathbf{u}_{h}\right|_{(\mathbf{u}, r, \alpha)} \leq\left[\Xi_{\alpha}\left(\left\|e\left(\mathbf{u}-\mathbf{u}_{h}\right)\right\|_{L^{r}(\Omega)}+\|e(\mathbf{u})\|_{L^{r}(\Omega)}\right)\right]^{\frac{r-2}{2}}\left\|e\left(\mathbf{u}-\mathbf{u}_{h}\right)\right\|_{L^{r}(\Omega)} .
$$


On considering

$$
\begin{array}{r}
\left\|e\left(\mathbf{u}-\mathbf{u}_{h}\right)\right\|_{L^{r}(\Omega)}+\|e(\mathbf{u})\|_{L^{r}(\Omega)} \leq 2\left(\left\|e\left(\mathbf{u}_{h}\right)\right\|_{L^{r}(\Omega)}+\|e(\mathbf{u})\|_{L^{r}(\Omega)}\right) \\
\leq 4 G^{-1}\left(\frac{1}{K_{1}}\|f\|_{\mathrm{V}^{\prime}}\right)
\end{array}
$$

we have

$$
\left|\mathbf{u}-\mathbf{u}_{h}\right|_{(\mathbf{u}, r, \alpha)} \leq 2^{r-2}\left[\Xi_{\alpha} \circ G^{-1}\left(\frac{1}{K_{1}}\|f\|_{\mathrm{V}^{\prime}}\right)\right]^{\frac{r-2}{2}}\left\|e\left(\mathbf{u}-\mathbf{u}_{h}\right)\right\|_{L^{r}(\Omega)} .
$$

Recalling the definition of $C_{4}$ we get

$$
\begin{array}{r}
\left\|\mathbf{S}_{1}\right\|_{\mathrm{V}^{\prime}} \leq C_{4}\left|\mathbf{u}-\mathbf{u}_{h}\right|_{(\mathbf{u}, r, \alpha)}+\left\|p-p_{h}\right\|_{\mathrm{Q}} \\
\leq C_{5}\left\|e\left(\mathbf{u}_{h}-\mathbf{u}\right)\right\|_{L^{r}(\Omega)}+\left\|p-p_{h}\right\|_{\mathrm{Q}}
\end{array}
$$

where $C_{5}=C_{3} 4^{r-2}\left[\Xi_{\alpha} \circ G^{-1}\left(\frac{1}{K_{1}}\|f\|_{\mathrm{V}^{\prime}}\right)\right]^{r-2}$.

The proof can be easily completed by bounding the norm of $S_{2}$ as follows

$$
\begin{aligned}
\left\|\mathrm{S}_{2}\right\|_{\mathrm{Q}^{\prime}} & =\sup _{q \in \mathrm{Q}} \frac{\left\langle\mathrm{S}_{2}, q\right\rangle}{\|q\|_{\mathrm{Q}}}=\sup _{q \in \mathrm{Q}} \frac{-b\left(q, \mathbf{u}_{h}\right)}{\|q\|_{\mathrm{Q}}}=\sup _{q \in \mathrm{Q}} \frac{b\left(q, \mathbf{u}-\mathbf{u}_{h}\right)}{\|q\|_{\mathrm{Q}}} \\
& =\left\|\nabla \cdot\left(\mathbf{u}-\mathbf{u}_{h}\right)\right\|_{\mathrm{L}^{r}(\Omega)} \leq\left\|e\left(\mathbf{u}-\mathbf{u}_{h}\right)\right\|_{\mathrm{L}^{r}(\Omega)}=\left\|\mathbf{u}-\mathbf{u}_{h}\right\|_{\mathrm{V}} .
\end{aligned}
$$

\subsection{General results}

Before proving our lower bounds in terms of computable residuals, we recall some results from [25] and [26]. Let us denote by $\mathrm{V}_{\hat{T}} \subset \mathrm{W}^{1, r}(\hat{T}) \cap \mathrm{L}^{\infty}(\hat{T})$ and $\mathrm{V}_{\hat{E}} \subset \mathrm{W}^{\frac{1}{r^{r}}, r}(\hat{E}) \cap \mathrm{L}^{\infty}(\hat{E})$ two arbitrary finite-dimensional spaces of functions defined over the reference element $\hat{T}$ and on the reference face $\hat{E}$, respectively, and let $\mathrm{V}_{T}=\left\{\hat{\phi} \circ F_{T}^{-1}: \hat{\phi} \in \mathrm{V}_{\hat{T}}\right\}$ and $\mathrm{V}_{E}=\left\{\hat{\phi} \circ F_{T}^{-1}: \hat{\phi} \in \mathrm{V}_{\hat{E}}, T \in \hat{\omega}_{E}\right\}$. The spaces $\mathrm{V}_{\hat{T}}$ and $\mathrm{V}_{\hat{E}}$ can be thought of as being related to the approximations introduced by suitable quadrature formulas; thus, they are supposed to be finite-dimensional (e.g. consisting of polynomials of a fixed degree). The theory below can be applied to more complex situations without significant changes.

Lemma 17 There are constants which only depend on the spaces $\mathrm{V}_{\hat{T}}$ and $\mathrm{V}_{\hat{E}}$, $r$ and the minimal angle of $T \in \mathcal{T}_{h}$ such that the following inequalities hold for all $w_{T} \in \mathrm{V}_{T}$ and all $w_{E} \in \mathrm{V}_{E}$ :

$$
\begin{aligned}
& \left\|w_{T}\right\|_{L^{r^{\prime}(T)}} \precsim \quad \sup _{v_{T} \in \mathrm{V}_{T}} \frac{\int_{T} w_{T} b_{T} v_{T} \mathrm{~d} \Omega}{\left\|b_{T} v_{T}\right\|_{L^{r}(T)}} \leq\left\|w_{T}\right\|_{L^{r^{\prime}(T)}}, \\
& \left\|w_{E}\right\|_{L^{r^{\prime}(E)}} \precsim \sup _{v_{E} \in \mathrm{V}_{E}} \frac{\int_{E} w_{E} \gamma_{E}\left(b_{E}\right) v_{E} \mathrm{~d} \Gamma}{\left\|b_{E} v_{E}\right\|_{L^{r}(E)}} \leq\left\|w_{T}\right\|_{L^{r^{\prime}(E)}}, \\
& h_{T}^{-1}\left\|b_{T} w_{T}\right\|_{L^{r}(T)} \precsim \quad\left\|\nabla\left(b_{T} w_{T}\right)\right\|_{L^{r}(T)} \quad \precsim h_{T}^{-1}\left\|b_{T} w_{T}\right\|_{L^{r}(T)}, \\
& h_{E}^{-1}\left\|b_{E} \mathcal{P}_{E}\left(w_{E}\right)\right\|_{L^{r}(T)} \precsim\left\|\nabla\left(b_{E} \mathcal{P}_{E}\left(w_{E}\right)\right)\right\|_{L^{r}(T)} \precsim h_{E}^{-1}\left\|b_{E} \mathcal{P}_{E}\left(w_{E}\right)\right\|_{L^{r}(T)}, \\
& \left\|b_{E} \mathcal{P}_{E}\left(w_{E}\right)\right\|_{L^{r}(T)} \precsim h_{E}^{\frac{1}{r}}\left\|w_{E}\right\|_{L^{r}(E)} .
\end{aligned}
$$


Here and below $\gamma_{E}\left(b_{E}\right)$ denotes the trace of $b_{E}$ on the face $E$.

Lemma 18 There are constants which only depend on the space $\mathrm{V}_{\hat{E}}, r$ and the minimal angle of $T \in \mathcal{T}_{h}$ such that the following inequality holds for all $w_{E} \in \mathrm{V}_{E}$ :

$$
\left\|\nabla\left(b_{E} \mathcal{P}_{E}\left(w_{E}\right)\right)\right\|_{L^{r}\left(\grave{\omega}_{E}\right)} \asymp h_{E}^{-\frac{1}{r^{\prime}}}\left\|w_{E}\right\|_{L^{r}(E)} .
$$

Proof. The relation

$$
\left\|\nabla\left(b_{E} \mathcal{P}_{E}\left(w_{E}\right)\right)\right\|_{L^{r}(T)} \precsim h_{E}^{-\frac{1}{r^{r}}}\left\|w_{E}\right\|_{L^{r}(E)}
$$

can be easily derived from (5.10) and (5.11), while the inequality

$$
h_{E}^{-\frac{1}{r^{r}}}\left\|w_{E}\right\|_{L^{r}(E)} \precsim\left\|\nabla\left(b_{E} \mathcal{P}_{E}\left(w_{E}\right)\right)\right\|_{L^{r}(T)}
$$

comes from the application of the trace inequality

$$
\left\|\gamma_{E}\left(b_{E}\right) w_{E}\right\|_{L^{r}(E)} \precsim h_{E}^{-\frac{1}{r}}\left\|b_{E} \mathcal{P}_{E}\left(w_{E}\right)\right\|_{L^{r}(T)}+h_{E}^{1-\frac{1}{r}}\left\|\nabla\left(b_{E} \mathcal{P}_{E}\left(w_{E}\right)\right)\right\|_{L^{r}(T)}
$$

together with (5.10) and the norm equivalence $\left\|\gamma_{E}\left(b_{E}\right) w_{E}\right\|_{L^{r}(E)} \asymp\left\|w_{E}\right\|_{L^{r}(E)}$. Then, the statement of the lemma follows from the regularity assumption for $\mathcal{T}_{h}$.

Any $v_{T} \in \mathrm{V}_{T}$ and $b_{T}$ will be assumed to be extended by 0 to $\bar{\Omega}$ outside $T$. Similarly, any $v_{E}$ will be assumed to be identically zero on $\mathcal{E}_{h, \Omega}$ outside $E$ and any $b_{E}$ will be

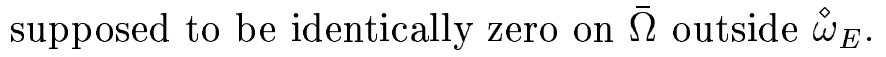

Let $v_{T} \in \mathrm{V}_{T}, v=\sum_{T \in \mathcal{T}_{h}} v_{T}$ and $b_{\mathcal{T}_{h}}=\sum_{T \in \mathcal{T}_{h}} b_{T}$; then, we define the following negative-order norms:

$$
\begin{aligned}
\|u\|_{\mathrm{W}^{-1, r^{\prime}}(T)} & =\sup _{v_{T} \in \mathrm{V}_{T}} \frac{\int_{T} u b_{T} v_{T} \mathrm{~d} \Omega}{\left\|b_{T} v_{T}\right\|_{\mathrm{W}^{1, r}(T)}}, \\
\|u\|_{\mathrm{W}^{-1, r^{\prime}}\left(\mathcal{T}_{h}\right)} & =\sup _{v \in \cup_{T \in \mathcal{T}_{h}} \mathrm{~V}_{T}} \frac{\int_{\Omega} u b_{\mathcal{T}_{h}} v \mathrm{~d} \Omega}{\left\|b_{\mathcal{T}_{h}} v\right\|_{\mathrm{W}^{1, r}(\Omega)}} .
\end{aligned}
$$

Similarly, let $v_{E} \in \mathrm{V}_{E}, v=\sum_{E \in \mathcal{E}_{h, \Omega}} \mathcal{P}_{E}\left(v_{E}\right)$ and $b_{\mathcal{E}_{h, \Omega}}=\sum_{E \in \mathcal{E}_{h, \Omega}} b_{E}$; then, we define

$$
\begin{aligned}
& \|u\|_{\mathrm{W}^{-\frac{1}{r^{,}, r^{\prime}}(E)}}=\sup _{v_{E} \in \mathrm{V}_{E}} \frac{\int_{E} u \gamma_{E}\left(b_{E}\right) v_{E} \mathrm{~d} \Gamma}{\left\|b_{E} \mathcal{P}_{E}\left(v_{E}\right)\right\|_{\mathrm{W}^{1, r}\left(\grave{\omega}_{E}\right)}} \\
& \|u\|_{\mathrm{W}^{-\frac{1}{r^{\prime}, r^{\prime}}}\left(\mathcal{E}_{h, \Omega}\right)}=\sup _{v \in \cup_{E \in \mathcal{E}_{h, \Omega} \mathrm{V}_{E}}} \frac{\sum_{E \in \mathcal{E}_{h, \Omega}} \int_{E} u \gamma_{E}\left(b_{\mathcal{E}_{h, \Omega}} v\right) \mathrm{d} \Gamma}{\left\|b_{\mathcal{E}_{h, \Omega}} v\right\|_{\mathrm{W}^{1, r}(\Omega)}} .
\end{aligned}
$$

Remark 19 The definition of the norm $\|\cdot\|_{\mathrm{W}^{-\frac{1}{r^{\prime}, r^{\prime}}(E)}}$ is motivated by the observation that

$$
h_{E}^{\frac{1}{r^{\prime}}}\|u\|_{L^{r^{\prime}}(E)}=\sup _{v_{E} \in \mathrm{L}^{r}(E)} \frac{\int_{E} u v_{E} \mathrm{~d} \Gamma}{h_{E}^{-\frac{1}{r^{\prime}}}\left\|v_{E}\right\|_{L^{r}(E)}}
$$


that for $v_{E} \in \mathrm{V}_{E} \subset \mathrm{L}^{r}(E)$, by (5.12) and the equivalence of $\|\cdot\|_{\mathrm{W}^{1, r}\left(\grave{\omega}_{E}\right)}$ to $|\cdot|_{\mathrm{W}^{1, r}\left(\grave{\omega}_{E}\right)}$ on

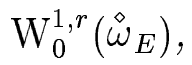

$$
h_{E}^{-\frac{1}{r^{\prime}}}\left\|v_{E}\right\|_{L^{r}(E)} \asymp\left\|b_{E} \mathcal{P}_{E}\left(v_{E}\right)\right\|_{\mathrm{W}^{1, r}\left(\hat{\omega}_{E}\right)},
$$

and that, for $u$ in a finite-dimensional subspace of $\mathrm{L}^{r^{\prime}}(E)$,

$$
h_{E}^{\frac{1}{r^{\prime}}}\|u\|_{L^{r^{\prime}}(E)} \asymp\|u\|_{\mathrm{W}^{-\frac{1}{r^{\prime}, r^{\prime}}}(E)} .
$$

Lemma 20 Let $u \in \mathrm{W}^{-1, r^{\prime}}(\Omega)$; then,

$$
\left(\sum_{T \in \mathcal{T}_{h}}\|u\|_{\mathrm{W}^{-1, r^{\prime}}(T)}^{r^{\prime}}\right)^{\frac{1}{r^{\prime}}} \leq\|u\|_{\mathrm{W}^{-1, r^{\prime}}\left(\mathcal{T}_{h}\right)} \leq\|u\|_{\mathrm{W}^{-1, r^{\prime}}(\Omega)} .
$$

Further, let $u$ be such that $\|u\|_{\mathrm{W}^{-\frac{1}{r^{\prime}, r^{\prime}}(E)}}<\infty$ for all $E$ in $\mathcal{E}_{h, \Omega}$; then

$$
\left(\sum_{E \in \mathcal{E}_{h, \Omega}}\|u\|_{\mathrm{W}^{-\frac{1}{r^{\prime}, r^{\prime}}}(E)}^{r^{\prime}}\right)^{\frac{1}{r^{\prime}}} \leq\|u\|_{\mathrm{W}^{-\frac{1}{r^{\prime}, r^{\prime}}}\left(\mathcal{E}_{h, \Omega}\right)} .
$$

Proof. To prove the first inequality in (5.17), let us consider an arbitrary $\varepsilon \in(0,1)$. By our definition of $\|u\|_{\mathrm{W}^{-1, r^{\prime}}(T)}$, for each $T$ there exists a $v_{T} \in \mathrm{V}_{T}$ with $\left\|b_{T} v_{T}\right\|_{\mathrm{W}^{1, r}(T)}=$ 1 such that

$$
\int_{T} u b_{T} v_{T} \mathrm{~d} \Omega \geq(1-\varepsilon)\|u\|_{\mathrm{W}^{-1, r^{\prime}}(T)} .
$$

Let us define

$$
v=\sum_{T \in \mathcal{T}_{h}}\|u\|_{\mathrm{W}^{-1, r^{\prime}}(T)}^{r^{\prime}-1} b_{T} v_{T} \in \mathrm{W}_{0}^{1, r}(\Omega) .
$$

It follows from these definitions that

$$
\begin{aligned}
\sum_{T \in \mathcal{T}_{h}}\|u\|_{\mathrm{W}^{-1, r^{\prime}}(T)}^{r^{\prime}} & \leq \frac{1}{1-\varepsilon} \sum_{T \in \mathcal{T}_{h}}\|u\|_{\mathrm{W}^{-1, r^{\prime}}(T)}^{r^{\prime}-1} \int_{T} u b_{T} v_{T} \mathrm{~d} \Omega \\
& =\frac{1}{1-\varepsilon} \int_{\Omega} u v \mathrm{~d} \Omega \\
& \leq \frac{1}{1-\varepsilon}\|u\|_{\mathrm{W}^{-1, r^{\prime}\left(\mathcal{T}_{h}\right)}\|v\|_{\mathrm{W}^{1, r}(\Omega)} .}
\end{aligned}
$$

Moreover,

$$
\begin{array}{r}
\|v\|_{\mathrm{W}^{1, r}(\Omega)}=\left(\sum_{T \in \mathcal{T}_{h}}\|v\|_{\mathrm{W}^{1, r}(T)}^{r}\right)^{\frac{1}{r}}=\left(\sum_{T \in \mathcal{T}_{h}}\|u\|_{\mathrm{W}^{-1, r^{\prime}}(T)}^{r\left(r^{\prime}-1\right)}\left\|b_{T} v_{T}\right\|_{\mathrm{W}^{1, r}(T)}^{r}\right)^{\frac{1}{r}} \\
=\left(\sum_{T \in \mathcal{T}_{h}}\|u\|_{\mathrm{W}^{-1, r^{\prime}}(T)}^{r\left(r^{\prime}-1\right)}\right)^{\frac{1}{r}} .
\end{array}
$$


If we substitute this relation into the previous one, observing that $r\left(r^{\prime}-1\right)=r^{\prime}$ and $1 / r=1-1 / r^{\prime}$ we get

$$
\left(\sum_{T \in \mathcal{T}_{h}}\|u\|_{\mathrm{W}^{-1, r^{\prime}}(T)}^{r^{\prime}}\right)^{\frac{1}{r^{\prime}}} \leq \frac{1}{1-\varepsilon}\|u\|_{\mathrm{W}^{-1, r^{\prime}}\left(\mathcal{T}_{h}\right)} .
$$

Since $\varepsilon \in(0,1)$ is arbitrary this proves the first inequality in (5.17). The second follows trivially from our definitions.

To prove the inequality (5.18), let us consider an arbitrary $\varepsilon \in(0,1)$. By our definition of $\|u\|_{\mathrm{W}^{-}{\frac{1}{r^{\prime}}}^{, r^{\prime}}(E)}$, for each $E \in \mathcal{E}_{h, \Omega}$ there exists a function $v_{E} \in \mathrm{V}_{E}$ with $\left\|b_{E} \mathcal{P}_{E}\left(v_{E}\right)\right\|_{\mathrm{W}^{1, r}\left(\stackrel{\leftrightarrow}{\omega}_{E}\right)}=1$ such that

$$
\int_{E} u \gamma_{E}\left(b_{E}\right) v_{E} \mathrm{~d} \Gamma \geq(1-\varepsilon)\|u\|_{\mathrm{W}^{-\frac{1}{r^{\prime}, r^{\prime}}}(E)}
$$

Let us define

$$
v=\sum_{E \in \mathcal{E}_{h, \Omega}}\|u\|_{\mathrm{W}^{-\frac{1}{r^{\prime}}, r^{\prime}}(E)}^{r^{\prime}-1} b_{E} \mathcal{P}_{E}\left(v_{E}\right) \in \mathrm{W}_{0}^{1, r}(\Omega) .
$$

It follows from our definitions that

$$
\begin{aligned}
\sum_{E \in \mathcal{E}_{h, \Omega}}\|u\|_{\mathrm{W}^{-\frac{1}{r^{\prime}, r^{\prime}}(E)}}^{r^{\prime}} & \leq \frac{1}{1-\varepsilon} \sum_{E \in \mathcal{E}_{h, \Omega}}\|u\|_{\mathrm{W}^{-\frac{1}{r^{\prime}, r^{\prime}}}(E)}^{r^{\prime}-1} \int_{E} u \gamma_{E}\left(b_{E}\right) v_{E} \mathrm{~d} \Gamma \\
& =\frac{1}{1-\varepsilon} \sum_{E \in \mathcal{E}_{h, \Omega}} \int_{E} u \gamma_{E}(v) \mathrm{d} \Gamma \\
& \leq \frac{1}{1-\varepsilon}\|u\|_{\mathrm{W}^{-\frac{1}{r^{\prime}}, r^{\prime}}\left(\mathcal{E}_{h, \Omega}\right)}\|v\|_{\mathrm{W}^{1, r}(\Omega)}
\end{aligned}
$$

Moreover, thanks to the orthogonality of the face bubble functions, we have

$$
\begin{array}{r}
\|v\|_{\mathrm{W}^{1, r}(\Omega)}=\left(\sum_{E \in \mathcal{E}_{h, \Omega}}\|v\|_{\mathrm{W}^{1, r}\left(\grave{\omega}_{E}\right)}^{r}\right)^{\frac{1}{r}}=\left(\sum_{E \in \mathcal{E}_{h, \Omega}}\|u\|_{\mathrm{W}^{-\frac{1}{r^{\prime}}, r^{\prime}(E)}}^{r\left(r^{\prime}-1\right)}\left\|b_{E} \mathcal{P}_{E}\left(v_{E}\right)\right\|_{\mathrm{W}^{1, r}\left(\grave{\omega}_{E}\right)}^{r}\right)^{\frac{1}{r}} \\
=\left(\sum_{E \in \mathcal{E}_{h, \Omega}}\|u\|_{\mathrm{W}^{-\frac{1}{r^{\prime}, r^{\prime}}(E)}}^{r\left(r^{\prime}-1\right)}\right)^{\frac{1}{r}} .
\end{array}
$$

If we substitute this relation into the previous one, on observing that $r\left(r^{\prime}-1\right)=r^{\prime}$ and $1 / r=1-1 / r^{\prime}$ we get

$$
\left(\sum_{E \in \mathcal{E}_{h}, \Omega}\|u\|_{\mathrm{W}^{-\frac{1}{r^{\prime}}, r^{\prime}}(E)}^{r^{\prime}}\right)^{\frac{1}{r^{\prime}}} \leq \frac{1}{1-\varepsilon}\|u\|_{\mathrm{W}^{-\frac{1}{r^{\prime}, r^{\prime}}}{ }_{\left(\mathcal{E}_{h, \Omega}\right)}} .
$$

Since $\varepsilon \in(0,1)$ is arbitrary, this proves the inequality (5.18). 


\subsection{Lower bound on the error in terms of computable residuals}

The results of using Propositions 22 and 23 and inequality (5.23) in this section have been collected in the following theorem:

Theorem 21 Let $(\mathbf{u}, p) \in \mathrm{V} \times \mathrm{Q}$ denote the solution to (1.4), (1.5), and let $\left(\mathbf{u}_{h}, p_{h}\right) \in$ $\mathrm{V}_{h} \times \mathrm{Q}_{h}$ denote its finite element approximation defined by (2.2), (2.3). Then, there exists a positive constant $c_{L}$ depending on $K_{1}, K_{2}, r,\|\mathbf{f}\|_{\mathrm{V}^{\prime}}$ and on the minimal angle of the triangulation such that

$$
\begin{aligned}
c_{L}\left[\left(\sum_{T \in \mathcal{T}_{h}} h_{T}^{r^{\prime}}\left\|\Pi_{T} \mathbf{R}_{T}\left(\left[\mathbf{u}_{h}, p_{h}\right]\right)\right\|_{L^{r^{\prime}}(T)}^{r^{\prime}}\right)^{\frac{\mathrm{R}_{\mathrm{L}}^{\prime}}{r^{\prime}}}\right. & +\left(\sum_{E \in \mathcal{E}_{h, \Omega}} h_{E}\left\|\Pi_{E} \mathbf{J}_{E}\left(\left[\mathbf{u}_{h}, p_{h}\right]\right)\right\|_{L^{r^{\prime}}(E)}^{r^{\prime}}\right)^{\frac{\mathrm{R}_{\mathrm{L}}^{\prime}}{r^{\prime}}} \\
& \left.+\left\|\nabla \cdot \mathbf{u}_{h}\right\|_{L^{r^{\prime}}(\Omega)}^{\mathrm{g}^{\prime}}\right] \leq\left\|\mathbf{u}-\mathbf{u}_{h}\right\|_{\mathrm{V}}^{\mathrm{R}_{\mathrm{L}}}+\left\|p-p_{h}\right\|_{\mathrm{Q}}^{\mathrm{g}} \\
& +\left(\sum_{T \in \mathcal{T}_{h}} h_{T}^{r^{\prime}}\left\|\mathbf{R}_{T}\left(\left[\mathbf{u}_{h}, p_{h}\right]\right)-\Pi_{T} \mathbf{R}_{T}\left(\left[\mathbf{u}_{h}, p_{h}\right]\right)\right\|_{L^{r^{\prime}}(T)}^{r^{\prime}}\right)^{\frac{\mathrm{R}_{\mathrm{L}}^{\prime}}{r^{\prime}}} \\
& +\left(\sum_{E \in \mathcal{E}_{h, \Omega}} h_{E}\left\|\mathbf{J}_{E}\left(\left[\mathbf{u}_{h}, p_{h}\right]\right)-\Pi_{E} \mathbf{J}_{E}\left(\left[\mathbf{u}_{h}, p_{h}\right]\right)\right\|_{L^{r^{\prime}}(E)}^{r^{\prime}}\right)^{\frac{\mathrm{R}_{\mathrm{L}}^{\prime}}{r^{\prime}}}
\end{aligned}
$$

where $\mathrm{R}_{\mathrm{L}}=\min \{r, 2\}, я=\max \left\{r^{\prime}, 2\right\}, 1 / \mathrm{R}_{\mathrm{L}}+1 / \mathrm{R}_{\mathrm{L}}^{\prime}=1,1 / \mathrm{s}+1 / \mathrm{s}^{\prime}=1$.

\subsubsection{Lower bound on the error in terms of the residual}

First, we state the lower bound on the error in terms of the element momentum residual.

Proposition 22 Under the hypotheses of Theorem 21,

$$
\begin{aligned}
\left(\sum_{T \in \mathcal{T}_{h}}\left\|h_{T} \Pi_{T} \mathbf{R}_{T}\left(\left[\mathbf{u}_{h}, p_{h}\right]\right)\right\|_{L^{r^{\prime}}(T)}^{r^{\prime}}\right)^{\frac{\mathrm{R}_{\mathrm{L}}^{\prime}}{r^{\prime}}} \precsim\left\|\mathbf{u}-\mathbf{u}_{h}\right\|_{\mathrm{V}}^{\mathrm{R}_{\mathrm{L}}}+\left\|p-p_{h}\right\|_{\mathrm{Q}}^{\mathrm{g}} \\
+\left(\sum_{T \in \mathcal{T}_{h}} h_{T}^{r^{\prime}}\left\|\mathbf{R}_{T}\left(\left[\mathbf{u}_{h}, p_{h}\right]\right)-\Pi_{T} \mathbf{R}_{T}\left(\left[\mathbf{u}_{h}, p_{h}\right]\right)\right\|_{L^{r^{\prime}}(T)}^{r^{\prime}}\right)^{\frac{\mathrm{R}_{\mathrm{L}}^{\prime}}{r^{\prime}}},
\end{aligned}
$$

where $\mathrm{R}_{\mathrm{L}}=\min \{r, 2\}$, я $=\max \left\{r^{\prime}, 2\right\}, 1 / \mathrm{R}_{\mathrm{L}}+1 / \mathrm{R}_{\mathrm{L}}^{\prime}=1,1 / \mathrm{s}+1 / \mathrm{g}^{\prime}=1$.

Proof. We begin by applying the vectorial analogue of the lower bound of (5.7) with $\mathbf{w}_{T}=h_{T} \Pi_{T} \mathbf{R}_{T}\left(\left[\mathbf{u}_{h}, p_{h}\right]\right)$ and of the upper bound of (5.9) with $\mathbf{w}_{T}=\mathbf{v}_{T}$, and noting that $\left\|\nabla\left(b_{T} \mathbf{v}_{T}\right)\right\|_{L^{r}(T)} \asymp\left\|b_{T} \mathbf{v}_{T}\right\|_{\mathrm{W}^{1, r}(T)}$; thus,

$$
\left\|h_{T} \Pi_{T} \mathbf{R}_{T}\left(\left[\mathbf{u}_{h}, p_{h}\right]\right)\right\|_{L^{r^{\prime}}(T)} \precsim \sup _{\mathbf{v}_{T} \in\left[\mathrm{V}_{T}\right]^{d}} \frac{\int_{T} h_{T} \Pi_{T} \mathbf{R}_{T}\left(\left[\mathbf{u}_{h}, p_{h}\right]\right) \cdot b_{T} \mathbf{v}_{T} \mathrm{~d} \Omega}{\left\|b_{T} \mathbf{v}_{T}\right\|_{L^{r}(T)}}
$$




$$
\begin{array}{r}
\precsim \sup _{\mathbf{v}_{T} \in\left[\mathrm{V}_{T}\right]^{d}} \frac{\int_{T} \Pi_{T} \mathbf{R}_{T}\left(\left[\mathbf{u}_{h}, p_{h}\right]\right) \cdot b_{T} \mathbf{v}_{T} \mathrm{~d} \Omega}{\left\|\nabla\left(b_{T} \mathbf{v}_{T}\right)\right\|_{L^{r}(T)}} \asymp \sup _{\mathbf{v}_{T} \in\left[\mathrm{V}_{T}\right]^{d}} \frac{\int_{T} \Pi_{T} \mathbf{R}_{T}\left(\left[\mathbf{u}_{h}, p_{h}\right]\right) \cdot b_{T} \mathbf{v}_{T} \mathrm{~d} \Omega}{\left\|b_{T} \mathbf{v}_{T}\right\|_{\mathrm{W}^{1, r}(T)}} \\
=\left\|\Pi_{T} \mathbf{R}_{T}\left(\left[\mathbf{u}_{h}, p_{h}\right]\right)\right\|_{\mathrm{W}^{-1, r^{\prime}(T)}}
\end{array}
$$

Now we apply Lemma 20 to deduce that

$$
\begin{aligned}
\left(\sum_{T \in \mathcal{T}_{h}}\left\|h_{T} \Pi_{T} \mathbf{R}_{T}\left(\left[\mathbf{u}_{h}, p_{h}\right]\right)\right\|_{L^{r^{\prime}}(T)}^{r^{\prime}}\right)^{\frac{1}{r^{\prime}}} & \precsim\left(\sum_{T \in \mathcal{T}_{h}}\left\|\Pi_{T} \mathbf{R}_{T}\left(\left[\mathbf{u}_{h}, p_{h}\right]\right)\right\|_{\mathrm{W}^{-1, r^{\prime}}(T)}^{r^{\prime}}\right)^{\frac{1}{r^{\prime}}} \\
& \leq\left\|\sum_{T \in \mathcal{T}_{h}} \Pi_{T} \mathbf{R}_{T}\left(\left[\mathbf{u}_{h}, p_{h}\right]\right)\right\| \|_{\mathrm{W}^{-1, r^{\prime}}\left(\mathcal{T}_{h}\right)}
\end{aligned}
$$

where we have used definition (5.14) with $\mathbf{v}=\sum_{T \in \mathcal{T}_{h}} \mathbf{v}_{T}$ and $b_{\mathcal{T}_{h}}=\sum_{T \in \mathcal{T}_{h}} b_{T}$.

Applying integration by parts and noting that each $b_{T}$ vanishes on $\partial T$, and using equations (1.4) and (1.5), we get:

$$
\begin{aligned}
& \left(\sum_{T \in \mathcal{T}_{h}}\left\|h_{T} \Pi_{T} \mathbf{R}_{T}\left(\left[\mathbf{u}_{h}, p_{h}\right]\right)\right\|_{L^{r^{\prime}(T)}}^{r^{\prime}}\right)^{\frac{1}{r^{\prime}}} \precsim \sup _{\mathbf{v} \in \cup_{T \in \mathcal{T}_{h}}\left[\mathrm{~V}_{T}\right]^{d}} \frac{\int_{\Omega} \sum_{T \in \mathcal{T}_{h}} \Pi_{T} \mathbf{R}_{T}\left(\left[\mathbf{u}_{h}, p_{h}\right]\right) \cdot b_{\mathcal{T}_{h}} \mathbf{v} \mathrm{d} \Omega}{\left\|b_{\mathcal{T}_{h}} \mathbf{v}\right\|_{\mathrm{W}^{1, r}(\Omega)}} \\
& \leq \sup _{\mathbf{v} \in \cup_{T \in \mathcal{T}_{h}}\left[\mathrm{~V}_{T}\right]^{d}} \frac{\int_{\Omega} \sum_{T \in \mathcal{T}_{h}} \mathbf{R}_{T}\left(\left[\mathbf{u}_{h}, p_{h}\right]\right) \cdot b_{\mathcal{T}_{h}} \mathbf{v} \mathrm{d} \Omega}{\left\|b_{\mathcal{T}_{h}} \mathbf{v}\right\|_{\mathrm{W}^{1, r}(\Omega)}}
\end{aligned}
$$

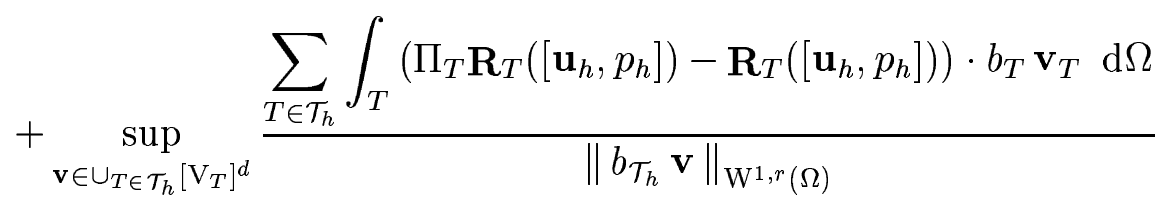

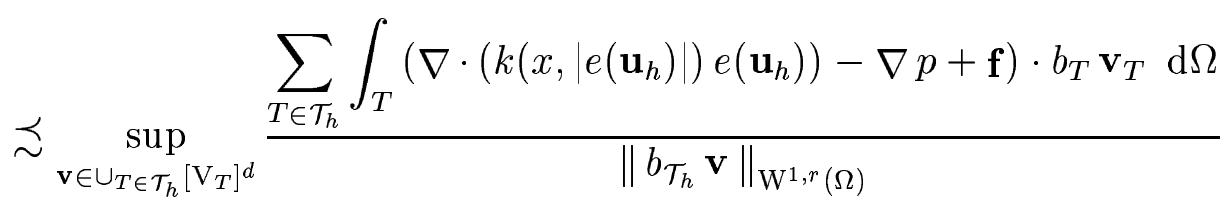

$$
\begin{aligned}
& +\sup _{\mathbf{v} \in \cup_{T \in \mathcal{T}_{h}}\left[\mathrm{~V}_{T}\right]^{d}} \frac{\sum_{T \in \mathcal{T}_{h}} \int_{T}\left(\Pi_{T} \mathbf{R}_{T}\left(\left[\mathbf{u}_{h}, p_{h}\right]\right)-\mathbf{R}_{T}\left(\left[\mathbf{u}_{h}, p_{h}\right]\right)\right) \cdot b_{T} \mathbf{v}_{T} \mathrm{~d} \Omega}{\left\|b_{\mathcal{T}_{h}} \mathbf{v}\right\|_{\mathrm{W}^{1, r}(\Omega)}}
\end{aligned}
$$

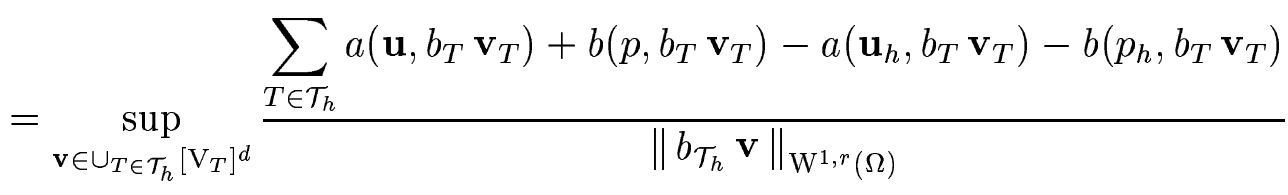

$$
\begin{aligned}
& +\sup _{\mathbf{v} \in \cup_{T \in \mathcal{T}_{h}}\left[\mathrm{~V}_{T}\right]^{d}} \frac{\sum_{T \in \mathcal{T}_{h}} \int_{T}\left(\Pi_{T} \mathbf{R}_{T}\left(\left[\mathbf{u}_{h}, p_{h}\right]\right)-\mathbf{R}_{T}\left(\left[\mathbf{u}_{h}, p_{h}\right]\right)\right) \cdot b_{T} \mathbf{v}_{T} \mathrm{~d} \Omega}{\left\|b_{\mathcal{T}_{h}} \mathbf{v}\right\|_{\mathrm{W}^{1, r}(\Omega)}}
\end{aligned}
$$




$$
\begin{array}{r}
\leq \sup _{\mathbf{v} \in \cup_{T \in \mathcal{T}_{h}}\left[\mathrm{~V}_{T}\right]^{d}} \frac{a\left(\mathbf{u}, b_{\mathcal{T}_{h}} \mathbf{v}\right)-a\left(\mathbf{u}_{h}, b_{\mathcal{T}_{h}} \mathbf{v}\right)}{\left\|b_{\mathcal{T}_{h}} \mathbf{v}\right\|_{\mathrm{W}^{1, r}(\Omega)}}+\sup _{\mathbf{v} \in \cup_{T \in \mathcal{T}_{h}}\left[\mathrm{~V}_{T}\right]^{d}} \frac{b\left(p-p_{h}, b_{\mathcal{T}_{h}} \mathbf{v}\right)}{\left\|b_{\mathcal{T}_{h}} \mathbf{v}\right\|_{\mathrm{W}^{1, r}(\Omega)}} \\
\quad+\sup _{\mathbf{v} \in \cup_{T \in \mathcal{T}_{h}}\left[\mathrm{~V}_{T}\right]^{d}} \frac{\sum_{T \in \mathcal{T}_{h}} \int_{T}\left(\Pi_{T} \mathbf{R}_{T}\left(\left[\mathbf{u}_{h}, p_{h}\right]\right)-\mathbf{R}_{T}\left(\left[\mathbf{u}_{h}, p_{h}\right]\right)\right) \cdot b_{T} \mathbf{v}_{T} \mathrm{~d} \Omega}{\left\|b_{\mathcal{T}_{h}} \mathbf{v}\right\|_{\mathrm{W}^{1, r}(\Omega)}} .
\end{array}
$$

The second term on the right-hand side can be bounded, by applying (5.1), in the following way:

$$
\begin{aligned}
\sup _{\mathbf{v} \in \cup_{T \in \mathcal{T}_{h}}\left[\mathrm{~V}_{T}\right]^{d}} \frac{b\left(p-p_{h}, b_{\mathcal{T}_{h}} \mathbf{v}\right)}{\left\|b_{\mathcal{T}_{h}} \mathbf{v}\right\|_{\mathrm{W}^{1, r}(\Omega)}} & \leq \sup _{\mathbf{v} \in \cup_{T \in \mathcal{T}_{h}\left[\mathrm{~V}_{T}\right]^{d}}} \frac{\left\|p-p_{h}\right\|_{\mathrm{Q}}\left\|\nabla \cdot\left(b_{\mathcal{T}_{h}} \mathbf{v}\right)\right\|_{\mathrm{L}^{r}(\Omega)}}{\left\|b_{\mathcal{T}_{h}} \mathbf{v}\right\|_{\mathrm{W}^{1, r}(\Omega)}} \\
& \leq\left\|p-p_{h}\right\|_{\mathrm{Q}} .
\end{aligned}
$$

The third term can be bounded by applying Hölder inequalities and the first inequality in (5.9) as follows:

$$
\begin{aligned}
& \frac{\sum_{T \in \mathcal{T}_{h}} \int_{T}\left(\Pi_{T} \mathbf{R}_{T}\left(\left[\mathbf{u}_{h}, p_{h}\right]\right)-\mathbf{R}_{T}\left(\left[\mathbf{u}_{h}, p_{h}\right]\right)\right) \cdot b_{T} \mathbf{v}_{T} \mathrm{~d} \Omega}{\left\|b_{T_{h}} \mathbf{v}\right\|} \\
& \sup _{\mathbf{v} \in \cup_{T \in \mathcal{T}_{h}}\left[\mathrm{~V}_{T}\right]^{d}}\|\| b_{\mathcal{T}_{h}} \mathbf{v} \|_{\mathrm{W}^{1, r}(\Omega)} \\
& \leq \sup _{\mathbf{v} \in \cup_{T \in \mathcal{T}_{h}}\left[\mathrm{~V}_{T}\right]^{d}} \frac{\sum_{T \in \mathcal{T}_{h}} h_{T}\left\|\Pi_{T} \mathbf{R}_{T}\left(\left[\mathbf{u}_{h}, p_{h}\right]\right)-\mathbf{R}_{T}\left(\left[\mathbf{u}_{h}, p_{h}\right]\right)\right\|_{L^{r^{\prime}}(\Omega)}\left\|h_{T}^{-1} b_{T} \mathbf{v}_{T}\right\|_{L^{r}(\Omega)}}{\left\|b_{\mathcal{T}_{h}} \mathbf{v}\right\|_{\mathrm{W}^{1, r}(\Omega)}} \\
& \leq \sup _{\mathbf{v} \in \cup_{T \in \mathcal{T}_{h}}\left[\mathrm{~V}_{T}\right]^{d}}\left(\sum_{T \in \mathcal{T}_{h}} h_{T}^{r^{\prime}}\left\|\Pi_{T} \mathbf{R}_{T}\left(\left[\mathbf{u}_{h}, p_{h}\right]\right)-\mathbf{R}_{T}\left(\left[\mathbf{u}_{h}, p_{h}\right]\right)\right\|_{L^{r^{\prime}}(\Omega)}^{r^{\prime}}\right)^{\frac{1}{r^{\prime}}} \\
& \times \frac{\left(\sum_{T \in \mathcal{T}_{h}}\left\|h_{T}^{-1} b_{T} \mathbf{v}_{T}\right\|_{\mathrm{L}^{r}(\Omega)}^{r}\right)^{\frac{1}{r}}}{\left\|b_{\mathcal{T}_{h}} \mathbf{v}\right\|_{\mathrm{W}^{1, r}(\Omega)}} .
\end{aligned}
$$

Next, we observe that (5.9) gives

$$
\begin{gathered}
\left(\sum_{T \in \mathcal{T}_{h}}\left\|h_{T}^{-1} b_{T} \mathbf{v}_{T}\right\|_{\mathrm{L}^{r}(T)}^{r}\right)^{\frac{1}{r}} \asymp\left(\sum_{T \in \mathcal{T}_{h}}\left\|\nabla\left(b_{T} \mathbf{v}_{T}\right)\right\|_{\mathrm{L}^{r}(T)}^{r}\right)^{\frac{1}{r}} \\
\asymp\left(\sum_{T \in \mathcal{T}_{h}}\left\|b_{T} \mathbf{v}_{T}\right\|_{\mathrm{W}^{1, r}(T)}^{r}\right)^{\frac{1}{r}}=\left(\left\|b_{\mathcal{T}_{h}} \mathbf{v}\right\|_{\mathrm{W}^{1, r}(\Omega)}^{r}\right)^{\frac{1}{r}}=\left\|b_{\mathcal{T}_{h}} \mathbf{v}\right\|_{\mathrm{W}^{1, r}(\Omega)}
\end{gathered}
$$

and thus we get

$$
\sup _{\mathbf{v} \in \cup_{T \in \mathcal{T}_{h}}\left[\mathrm{~V}_{T}\right]^{d}} \frac{\sum_{T \in \mathcal{T}_{h}} \int_{T}\left(\Pi_{T} \mathbf{R}_{T}\left(\left[\mathbf{u}_{h}, p_{h}\right]\right)-\mathbf{R}_{T}\left(\left[\mathbf{u}_{h}, p_{h}\right]\right)\right) \cdot b_{T} \mathbf{v}_{T} \mathrm{~d} \Omega}{\left\|b_{\mathcal{T}_{h}} \mathbf{v}\right\|_{\mathrm{W}^{1, r}(\Omega)}}
$$




$$
\precsim\left(\sum_{T \in \mathcal{T}_{h}} h_{T}^{r^{\prime}}\left\|\Pi_{T} \mathbf{R}_{T}\left(\left[\mathbf{u}_{h}, p_{h}\right]\right)-\mathbf{R}_{T}\left(\left[\mathbf{u}_{h}, p_{h}\right]\right)\right\|_{L^{r^{\prime}}(\Omega)}^{r^{\prime}}\right)^{\frac{1}{r^{\prime}}} .
$$

Now, we apply (3.5), (5.3), (5.21) and the last inequality to deduce that

$$
\begin{array}{r}
\left(\sum_{T \in \mathcal{T}_{h}}\left\|h_{T} \Pi_{T} \mathbf{R}_{T}\left(\left[\mathbf{u}_{h}, p_{h}\right]\right)\right\|_{L^{r^{\prime}}(T)}^{r^{\prime}}\right)^{\frac{1}{r^{\prime}}} \precsim C_{4}\left|\mathbf{u}-\mathbf{u}_{h}\right|_{(\mathbf{u}, r, \alpha)}^{\min \left\{1, \frac{2}{r^{\prime}}\right\}}+\left\|p-p_{h}\right\|_{\mathrm{Q}} \\
+\left(\sum_{T \in \mathcal{T}_{h}} h_{T}^{r^{\prime}}\left\|\Pi_{T} \mathbf{R}_{T}\left(\left[\mathbf{u}_{h}, p_{h}\right]\right)-\mathbf{R}_{T}\left(\left[\mathbf{u}_{h}, p_{h}\right]\right)\right\|_{L^{r^{\prime}}(\Omega)}^{r^{\prime}}\right)^{\frac{1}{r^{\prime}}} .
\end{array}
$$

The inequality (5.20) can be obtained by an argument similar to the one used in the proof of Theorem 16 after equation (5.6).

\subsubsection{Lower bound on the error in terms of the divergence of the velocity} Applying equations (1.5) and (5.2) we get

$$
\begin{aligned}
\left\|\nabla \cdot \mathbf{u}_{h}\right\|_{L^{r}(\Omega)} & =\left\|\nabla \cdot\left(\mathbf{u}_{h}-\mathbf{u}\right)\right\|_{L^{r}(\Omega)} \\
& \leq\left\|e\left(\mathbf{u}-\mathbf{u}_{h}\right)\right\|_{L^{r}(\Omega)}=\left\|\mathbf{u}-\mathbf{u}_{h}\right\|_{\mathrm{V}} .
\end{aligned}
$$

\subsubsection{Lower bound on the error in terms of the jumps}

Next, we show how the jumps $\mathbf{J}_{E}\left(\left[\mathbf{u}_{h}, p_{h}\right]\right)$ on each internal element-face $E$ bound the error from below.

Proposition 23 Under the hypotheses of Theorem 21,

$$
\begin{gathered}
\left(\sum_{E \in \mathcal{E}_{h, \Omega}} h_{E}\left\|\Pi_{E} \mathbf{J}_{E}\left(\left[\mathbf{u}_{h}, p_{h}\right]\right)\right\|_{L^{r^{\prime}(E)}}^{r^{\prime}}\right)^{\frac{\mathrm{R}_{\mathrm{L}}^{\prime}}{r^{\prime}}} \precsim\left\|e\left(\mathbf{u}-\mathbf{u}_{h}\right)\right\|_{L^{r}(\Omega)}^{\mathrm{R}_{\mathrm{L}}}+\left\|p-p_{h}\right\|_{L^{r^{\prime}(\Omega)}}^{\mathrm{g}} \\
+\left(\sum_{E \in \mathcal{E}_{h}, \Omega} h_{E}\left\|\mathbf{J}_{E}\left(\left[\mathbf{u}_{h}, p_{h}\right]\right)-\Pi_{E} \mathbf{J}_{E}\left(\left[\mathbf{u}_{h}, p_{h}\right]\right)\right\|_{L^{r^{\prime}(E)}}^{r^{\prime}}\right)^{\frac{\mathrm{R}_{L}^{\prime}}{r^{\prime}}}
\end{gathered}
$$

where $\mathrm{R}_{\mathrm{L}}=\min \{r, 2\}, я=\max \left\{r^{\prime}, 2\right\}, 1 / \mathrm{R}_{\mathrm{L}}+1 / \mathrm{R}_{\mathrm{L}}^{\prime}=1,1 / \mathrm{s}+1 / \mathrm{g}^{\prime}=1$.

Proof. We begin by applying the lower bound from (5.8) in which we use $w_{E}=$

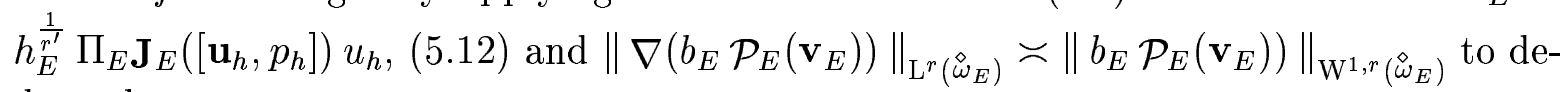
duce that

$$
\begin{aligned}
\left\|h_{E}^{\frac{1}{r^{\prime}}} \Pi_{E} \mathbf{J}_{E}\left(\left[\mathbf{u}_{h}, p_{h}\right]\right)\right\|_{L^{r^{\prime}}(E)} \precsim \sup _{\mathbf{v}_{E} \in\left[\mathrm{V}_{E}\right]^{d}} \frac{\int_{E} \Pi_{E} \mathbf{J}_{E}\left(\left[\mathbf{u}_{h}, p_{h}\right]\right) \cdot \gamma_{E}\left(b_{E}\right) \mathbf{v}_{E} \mathrm{~d} \Gamma}{\left\|\nabla\left(b_{E} \mathcal{P}_{E}\left(\mathbf{v}_{E}\right)\right)\right\|_{\mathrm{L}^{r}\left(\grave{\omega}_{E}\right)}} \\
\precsim \sup _{\mathbf{v}_{E} \in\left[\mathrm{V}_{E}\right]^{d}} \frac{\int_{E} \Pi_{E} \mathbf{J}_{E}\left(\left[\mathbf{u}_{h}, p_{h}\right]\right) \cdot \gamma_{E}\left(b_{E}\right) \mathbf{v}_{E} \mathrm{~d} \Gamma}{\left\|b_{E} \mathcal{P}_{E}\left(\mathbf{v}_{E}\right)\right\|_{\mathrm{W}^{1, r}\left(\grave{\omega}_{E}\right)}}=\left\|\Pi_{E} \mathbf{J}_{E}\left(\left[\mathbf{u}_{h}, p_{h}\right]\right)\right\|_{\mathrm{W}^{-\frac{1}{r^{\prime}, r^{\prime}}(E)}} .
\end{aligned}
$$


Now we apply definition (5.15) and Lemma 20 to get that

$$
\begin{aligned}
& \left(\sum_{E \in \mathcal{E}_{h, \Omega}}\left\|h_{E}^{\frac{1}{r^{\prime}}} \mathbf{J}_{E}\left(\left[\mathbf{u}_{h}, p_{h}\right]\right)\right\|_{L^{r^{\prime}(E)}}^{r^{\prime}}\right)^{\frac{1}{r^{\prime}}} \precsim\left(\sum_{E \in \mathcal{E}_{h, \Omega}}\left\|\mathbf{J}_{E}\left(\left[\mathbf{u}_{h}, p_{h}\right]\right)\right\|_{\mathrm{W}^{-\frac{1}{r^{\prime}, r^{\prime}}(E)}}^{r^{\prime}}\right)^{\frac{1}{r^{\prime}}} \\
& \leq\left\|\sum_{E \in \mathcal{E}_{h, \Omega}} \mathbf{J}_{E}\left(\left[\mathbf{u}_{h}, p_{h}\right]\right)\right\| \|_{\mathrm{W}^{-\frac{1}{r^{\prime}, r^{\prime}}}\left(\mathcal{E}_{h, \Omega}\right)},
\end{aligned}
$$

where we used definition (5.16) with $\mathbf{v}=\sum_{E \in \mathcal{E}_{h, \Omega}} \mathcal{P}_{E}\left(\mathbf{v}_{E}\right)$ and $b_{\mathcal{E}_{h, \Omega}}=\sum_{E \in \mathcal{E}_{h, \Omega}} b_{E}$.

Hence, on applying integration by parts and noting that $\operatorname{supp} b_{E} \subset \grave{\omega}_{E}$ and vanishes on all the faces of the element $T \in \dot{\omega}_{E} \subset \mathcal{T}_{h}^{\diamond}$ different from $E$, so the trace $\gamma_{\partial T}\left(b_{E} \mathbf{v}_{E}\right)$ is non-zero only on $E$, we get:

$$
\begin{aligned}
& \left(\sum_{E \in \mathcal{E}_{h, \Omega}}\left\|h_{E}^{\frac{1}{r^{\prime}}} \Pi_{E} \mathbf{J}_{E}\left(\left[\mathbf{u}_{h}, p_{h}\right]\right)\right\|_{L^{r^{\prime}}(E)}^{r^{\prime}}\right)^{\frac{1}{r^{\prime}}} \\
& \precsim \sup _{v \in \cup_{E \in \mathcal{E}_{h, \Omega}}\left[\mathrm{V}_{E}\right]^{d}} \frac{\sum_{E \in \mathcal{E}_{h, \Omega}} \int_{E} \mathbf{J}_{E}\left(\left[\mathbf{u}_{h}, p_{h}\right]\right) \cdot \gamma_{E}\left(b_{\mathcal{E}_{h, \Omega}} \mathbf{v}\right) \mathrm{d} \Gamma}{\left\|b_{\mathcal{E}_{h, \Omega}} \mathbf{v}\right\|_{\mathrm{W}^{1, r}(\Omega)}} \\
& +\sup _{v \in \cup_{E \in \mathcal{E}_{h, \Omega}}\left[\mathrm{V}_{E}\right]^{d}} \frac{\sum_{E \in \mathcal{E}_{h, \Omega}} \int_{E}\left(\Pi_{E} \mathbf{J}_{E}\left(\left[\mathbf{u}_{h}, p_{h}\right]\right)-\mathbf{J}_{E}\left(\left[\mathbf{u}_{h}, p_{h}\right]\right)\right) \cdot \gamma_{E}\left(b_{\mathcal{E}_{h, \Omega}} \mathbf{v}\right) \mathrm{d} \Gamma}{\left\|b_{\mathcal{E}_{h, \Omega}} \mathbf{v}\right\|_{\mathrm{W}^{1, r}(\Omega)}} \\
& =\sup _{\mathbf{v} \in \cup_{E \in \mathcal{E}_{h, \Omega}}\left[\mathrm{V}_{E}\right]^{d}} \frac{\sum_{T \in \mathcal{T}_{h}^{\diamond}} \int_{\partial T} \gamma_{\partial T}\left(\hat{n}_{E} \cdot\left(k\left(x,\left|e\left(\mathbf{u}_{h}\right)\right|\right) e\left(\mathbf{u}_{h}\right)-p_{h} \mathbf{I}\right)\right) \cdot \gamma_{\partial T}\left(b_{\mathcal{E}_{h, \Omega}} \mathbf{v}\right) \mathrm{d} \Omega}{\left\|b_{\mathcal{E}_{h, \Omega}} \mathbf{v}\right\|_{\mathrm{W}^{1, r}(\Omega)}} \\
& +\sup _{v \in \cup_{E \in \mathcal{E}_{h, \Omega}}\left[\mathrm{V}_{E}\right]^{d}} \frac{\sum_{E \in \mathcal{E}_{h, \Omega}} \int_{E}\left(\Pi_{E} \mathbf{J}_{E}\left(\left[\mathbf{u}_{h}, p_{h}\right]\right)-\mathbf{J}_{E}\left(\left[\mathbf{u}_{h}, p_{h}\right]\right)\right) \cdot \gamma_{E}\left(b_{\mathcal{E}_{h, \Omega}} \mathbf{v}\right) \mathrm{d} \Gamma}{\left\|b_{\mathcal{E}_{h, \Omega}} \mathbf{v}\right\|_{\mathrm{W}^{1, r}(\Omega)}} \\
& =\sup _{\mathbf{v} \in \cup_{E \in \mathcal{E}_{h, \Omega}}\left[\mathrm{V}_{E}\right]^{d}} \frac{\sum_{T \in \mathcal{T}_{h}^{\diamond}} \int_{T} \nabla \cdot\left(\left(k\left(x,\left|e\left(\mathbf{u}_{h}\right)\right|\right) e\left(\mathbf{u}_{h}\right)-p_{h} \mathbf{I}\right) b_{\mathcal{E}_{h, \Omega}} \mathbf{v}\right) \mathrm{d} \Omega}{\left\|b_{\mathcal{E}_{h, \Omega}} \mathbf{v}\right\|_{\mathrm{W}^{1, r}(\Omega)}} \\
& +\sup _{v \in \cup_{E \in \mathcal{E}_{h, \Omega}}\left[\mathrm{V}_{E}\right]^{d}} \frac{\sum_{E \in \mathcal{E}_{h, \Omega}} \int_{E}\left(\Pi_{E} \mathbf{J}_{E}\left(\left[\mathbf{u}_{h}, p_{h}\right]\right)-\mathbf{J}_{E}\left(\left[\mathbf{u}_{h}, p_{h}\right]\right)\right) \cdot \gamma_{E}\left(b_{\mathcal{E}_{h, \Omega}} \mathbf{v}\right) \mathrm{d} \Gamma}{\left\|b_{\mathcal{E}_{h, \Omega}} \mathbf{v}\right\|_{\mathrm{W}^{1, r}(\Omega)}}
\end{aligned}
$$




$$
\begin{aligned}
& =\sup _{\mathbf{v} \in \cup_{E \in \mathcal{E}_{h, \Omega}}\left[\mathrm{V}_{E}\right]^{d}}\left[\frac{\sum_{T \in \mathcal{T}_{h}^{\diamond}} \int_{T} \nabla \cdot\left(\left(k\left(x,\left|e\left(\mathbf{u}_{h}\right)\right|\right) e\left(\mathbf{u}_{h}\right)-p_{h} \mathbf{I}\right)\right) \cdot\left(b_{\mathcal{E}_{h, \Omega}} \mathbf{v}\right) \mathrm{d} \Omega}{\left\|b_{\mathcal{E}_{h, \Omega}} \mathbf{v}\right\|_{\mathrm{W}^{1, r}(\Omega)}}\right. \\
& \left.+\frac{\sum_{T \in \mathcal{T}_{h}^{\diamond}} \int_{T} \mathbf{f} \cdot b_{\mathcal{E}_{h, \Omega}} \mathbf{v} \mathrm{d} \Omega}{\left\|b_{\mathcal{E}_{h, \Omega}} \mathbf{v}\right\|_{\mathrm{W}^{1, r}(\Omega)}}\right] \\
& +\sup _{\mathbf{v} \in \cup_{E \in \mathcal{E}_{h, \Omega}}\left[\mathrm{V}_{E}\right]^{d}}\left[\frac{\sum_{T \in \mathcal{T}_{h}^{\diamond}} \int_{T}\left(\left(k\left(x,\left|e\left(\mathbf{u}_{h}\right)\right|\right) e\left(\mathbf{u}_{h}\right)-p_{h} \mathbf{I}\right)\right): \nabla\left(b_{\left.\mathcal{E}_{h, \Omega} \mathbf{v}\right) \mathrm{d} \Omega}\right.}{\left\|b_{\mathcal{E}_{h, \Omega}} \mathbf{v}\right\|_{\mathrm{W}^{1, r}(\Omega)}}\right. \\
& \left.-\frac{\sum_{T \in \mathcal{T}_{h}^{\diamond}} \int_{T} \mathbf{f} \cdot b_{\mathcal{E}_{h, \Omega}} \mathbf{v} \mathrm{d} \Omega}{\left\|b_{\mathcal{E}_{h, \Omega}} \mathbf{v}\right\|_{\mathrm{W}^{1, r}(\Omega)}}\right] \\
& +\sup _{v \in \cup_{E \in \mathcal{E}_{h, \Omega}}\left[\mathrm{V}_{E}\right]^{d}} \frac{\sum_{E \in \mathcal{E}_{h, \Omega}} \int_{E}\left(\Pi_{E} \mathbf{J}_{E}\left(\left[\mathbf{u}_{h}, p_{h}\right]\right)-\mathbf{J}_{E}\left(\left[\mathbf{u}_{h}, p_{h}\right]\right)\right) \cdot \gamma_{E}\left(b_{\mathcal{E}_{h, \Omega}} \mathbf{v}\right) \mathrm{d} \Gamma}{\left\|b_{\mathcal{E}_{h, \Omega}} \mathbf{v}\right\|_{\mathrm{W}^{1, r}(\Omega)}} .
\end{aligned}
$$

After applying equation (1.4) we get

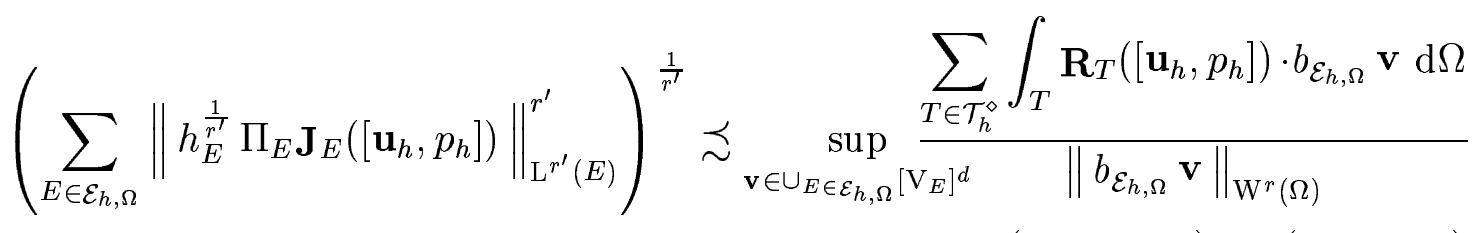

$$
\begin{aligned}
& +\sup _{\mathbf{v} \in \cup_{E \in \mathcal{E}_{h, \Omega}}\left[\mathrm{V}_{E}\right]^{d}} \frac{a\left(\mathbf{u}_{h}, b_{\mathcal{E}_{h, \Omega}} \mathbf{v}\right)-a\left(\mathbf{u}, b_{\mathcal{E}_{h, \Omega}} \mathbf{v}\right)}{\left\|b_{\mathcal{E}_{h, \Omega}} \mathbf{v}\right\|_{\mathrm{W}^{1, r}(\Omega)}} \\
& +\sup _{v \in \cup_{E \in \mathcal{E}_{h, \Omega}}\left[\mathrm{V}_{E}\right]^{d}} \frac{b\left(p_{h}-p, b_{\mathcal{E}_{h, \Omega}} \mathbf{v}\right)}{\left\|b_{\mathcal{E}_{h, \Omega}} \mathbf{v}\right\|_{\mathrm{W}^{1, r}(\Omega)}} \\
& +\sup _{v \in \cup_{E \in \mathcal{E}_{h, \Omega}}\left[\mathrm{V}_{E}\right]^{d}} \frac{\sum_{E \in \mathcal{E}_{h, \Omega}} \int_{E}\left(\Pi_{E} \mathbf{J}_{E}\left(\left[\mathbf{u}_{h}, p_{h}\right]\right)-\mathbf{J}_{E}\left(\left[\mathbf{u}_{h}, p_{h}\right]\right)\right) \cdot \gamma_{E}\left(b_{\mathcal{E}_{h, \Omega}} \mathbf{v}\right) \mathrm{d} \Gamma}{\left\|b_{\mathcal{E}_{h, \Omega}} \mathbf{v}\right\|_{\mathrm{W}^{1, r}(\Omega)}} .
\end{aligned}
$$

Now, write the integral over $\Omega$ in the first term of (5.25) as a sum of integrals over the elements $T$, then apply Hölder's inequality first to the integral over $T$ and then to the 
sum over $\mathcal{T}_{h}$ to get

$$
\begin{array}{r}
\sum_{T \in \mathcal{T}_{h}^{\circ}} \int_{T} \mathbf{R}_{T}\left(\left[\mathbf{u}_{h}, p_{h}\right]\right) \cdot b_{\mathcal{E}_{h, \Omega}} \mathbf{v} \mathrm{d} \Omega=\sum_{T \in \mathcal{T}_{h}} \int_{T} \mathbf{R}_{T}\left(\left[\mathbf{u}_{h}, p_{h}\right]\right) \cdot b_{\mathcal{E}_{h, \Omega}} \mathbf{v} \mathrm{d} \Omega \\
=\sum_{T \in \mathcal{T}_{h}} \int_{T} \Pi_{T} \mathbf{R}_{T}\left(\left[\mathbf{u}_{h}, p_{h}\right]\right) \cdot b_{\mathcal{E}_{h, \Omega}} \mathbf{v} \mathrm{d} \Omega \\
+\sum_{T \in \mathcal{T}_{h}} \int_{T}\left(\mathbf{R}_{T}\left(\left[\mathbf{u}_{h}, p_{h}\right]\right)-\Pi_{T} \mathbf{R}_{T}\left(\left[\mathbf{u}_{h}, p_{h}\right]\right)\right) \cdot b_{\mathcal{E}_{h, \Omega}} \mathbf{v} \mathrm{d} \Omega \\
\leq \sum_{T \in \mathcal{T}_{h}}\left\|h_{T} \Pi_{T} \mathbf{R}_{T}\left(\left[\mathbf{u}_{h}, p_{h}\right]\right)\right\|_{L^{r^{\prime}}(T)}\left\|h_{T}^{-1} b_{\mathcal{E}_{h, \Omega}} \mathbf{v}\right\|_{L^{r}(T)} \\
+\sum_{T \in \mathcal{T}_{h}}\left\|h_{T}\left(\mathbf{R}_{T}\left(\left[\mathbf{u}_{h}, p_{h}\right]\right)-\Pi_{T} \mathbf{R}_{T}\left(\left[\mathbf{u}_{h}, p_{h}\right]\right)\right)\right\|_{L^{r^{\prime}}(T)}\left\|h_{T}^{-1} b_{\mathcal{E}_{h, \Omega}} \mathbf{v}\right\|_{L^{r}(T)} \\
\leq\left(\sum_{T \in \mathcal{T}_{h}}\left\|h_{T} \Pi_{T} \mathbf{R}_{T}\left(\left[\mathbf{u}_{h}, p_{h}\right]\right)\right\|_{L^{r^{\prime}}(T)}^{r^{\prime}}\right)^{\frac{1}{r}}\left(\sum_{T \in \mathcal{T}_{h}}\left\|h_{T}^{-1} b_{\mathcal{E}_{h, \Omega}} \mathbf{v}\right\|_{L^{r}(T)}^{r}\right)^{\frac{1}{r}} \\
+\left(\sum_{T \in \mathcal{T}_{h}}\left\|h_{T}\left(\mathbf{R}_{T}\left(\left[\mathbf{u}_{h}, p_{h}\right]\right)-\Pi_{T} \mathbf{R}_{T}\left(\left[\mathbf{u}_{h}, p_{h}\right]\right)\right)\right\|_{L^{r^{\prime}}(T)}^{r^{\prime}}\right)^{\frac{1}{r^{\prime}}}\left(\sum_{T \in \mathcal{T}_{h}}\left\|h_{T}^{-1} b_{\mathcal{E}_{h, \Omega}} \mathbf{v}\right\|_{L^{r}(T)}^{r}\right)^{\frac{1}{r}} .
\end{array}
$$

Next, we observe that (5.9) gives

$$
\begin{array}{r}
\left(\sum_{T \in \mathcal{T}_{h}}\left\|h_{T}^{-1} b_{\mathcal{E}_{h, \Omega}} \mathbf{v}\right\|_{\mathrm{L}^{r}(T)}^{r}\right)^{\frac{1}{r}} \asymp\left(\sum_{T \in \mathcal{T}_{h}}\left\|\nabla\left(b_{\mathcal{E}_{h, \Omega}} \mathbf{v}\right)\right\|_{\mathrm{L}^{r}(T)}^{r}\right)^{\frac{1}{r}} \\
\asymp\left(\sum_{T \in \mathcal{T}_{h}}\left\|b_{\mathcal{E}_{h, \Omega}} \mathbf{v}\right\|_{\mathrm{W}^{1, r}(T)}^{r}\right)^{\frac{1}{r}}=\left(\left\|b_{\mathcal{E}_{h, \Omega}} \mathbf{v}\right\|_{\mathrm{W}^{1, r}(\Omega)}^{r}\right)^{\frac{1}{r}}=\left\|b_{\mathcal{E}_{h, \Omega}} \mathbf{v}\right\|_{\mathrm{W}^{1, r}(\Omega)}
\end{array}
$$

and deduce that

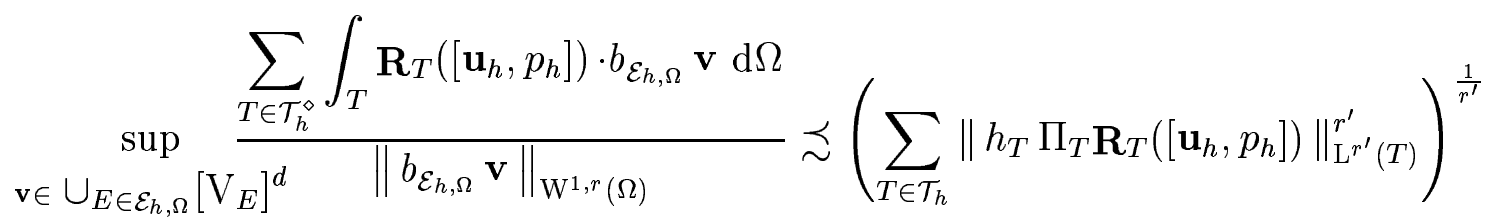

$$
\begin{aligned}
& +\left(\sum_{T \in \mathcal{T}_{h}}\left\|h_{T}\left(\mathbf{R}_{T}\left(\left[\mathbf{u}_{h}, p_{h}\right]\right)-\Pi_{T} \mathbf{R}_{T}\left(\left[\mathbf{u}_{h}, p_{h}\right]\right)\right)\right\|_{L^{r^{\prime}}(T)}^{r^{\prime}}\right)^{\frac{1}{r^{\prime}}} .
\end{aligned}
$$

Moreover, applying Hölder inequalities and equation (5.12) we get

$$
\frac{\sum_{E \in \mathcal{E}_{h, \Omega}} \int_{E}\left(\Pi_{E} \mathbf{J}_{E}\left(\left[\mathbf{u}_{h}, p_{h}\right]\right)-\mathbf{J}_{E}\left(\left[\mathbf{u}_{h}, p_{h}\right]\right)\right) \cdot \gamma_{E}\left(b_{\mathcal{E}_{h, \Omega}} \mathbf{v}\right) \mathrm{d} \Gamma}{\left\|b_{\mathcal{E}_{h, \Omega}} \mathbf{v}\right\|_{\mathrm{W}^{1, r}(\Omega)}}
$$




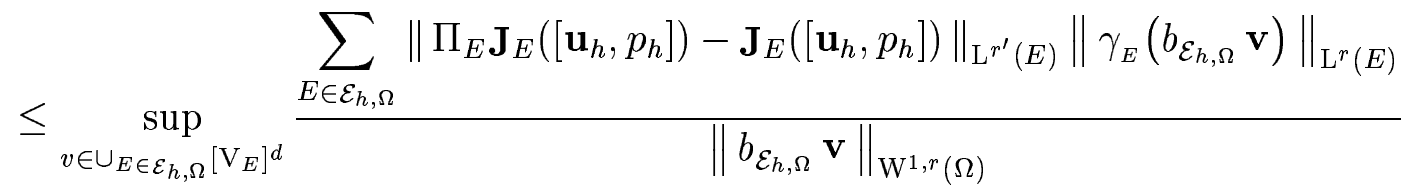

$$
\begin{aligned}
& \asymp \sup _{v \in \cup_{E \in \mathcal{E}_{h, \Omega}}\left[\mathrm{V}_{E}\right]^{d}} \frac{\sum_{E \in \mathcal{E}_{h, \Omega}} h_{E}^{\frac{1}{r^{\prime}}}\left\|\Pi_{E} \mathbf{J}_{E}\left(\left[\mathbf{u}_{h}, p_{h}\right]\right)-\mathbf{J}_{E}\left(\left[\mathbf{u}_{h}, p_{h}\right]\right)\right\|_{L^{r^{\prime}}(E)}\left\|b_{\mathcal{E}_{h, \Omega}} \mathbf{v}\right\|_{\mathrm{W}^{1, r}\left(\stackrel{\leftrightarrow}{\omega}_{E}\right)}}{\left\|b_{\mathcal{E}_{h, \Omega}} \mathbf{v}\right\|_{\mathrm{W}^{1, r}(\Omega)}} \\
& \leq\left(\sum_{E \in \mathcal{E}_{h, \Omega}} h_{E}\left\|\mathbf{J}_{E}\left(\left[\mathbf{u}_{h}, p_{h}\right]\right)-\Pi_{E} \mathbf{J}_{E}\left(\left[\mathbf{u}_{h}, p_{h}\right]\right)\right\|_{L^{r^{\prime}(E)}}^{r^{\prime}}\right)^{\frac{1}{r^{\prime}}}
\end{aligned}
$$

Now, we apply (3.5) to the second term of (5.25) and the same argument as the one used in (5.21) to the third term in (5.25) to deduce that

$$
\begin{array}{r}
\left(\sum_{E \in \mathcal{E}_{h, \Omega}} h_{E}\left\|\Pi_{E} \mathbf{J}_{E}\left(\left[\mathbf{u}_{h}, p_{h}\right]\right)\right\|_{L^{r^{\prime}}(E)}^{r^{\prime}}\right)^{\frac{1}{r^{\prime}}} \precsim\left(\sum_{T \in \mathcal{T}_{h}}\left\|h_{T} \Pi_{T} \mathbf{R}_{T}\left(\left[\mathbf{u}_{h}, p_{h}\right]\right)\right\|_{L^{r^{\prime}(T)}}^{r^{\prime}}\right)^{\frac{1}{r^{\prime}}} \\
+\left(\sum_{T \in \mathcal{T}_{h}}\left\|h_{T}\left(\mathbf{R}_{T}\left(\left[\mathbf{u}_{h}, p_{h}\right]\right)-\Pi_{T} \mathbf{R}_{T}\left(\left[\mathbf{u}_{h}, p_{h}\right]\right)\right)\right\|_{L^{r^{\prime}(T)}}^{r^{\prime}}\right)^{\frac{1}{r^{\prime}}} \\
+\left(\sum_{E \in \mathcal{E}_{h, \Omega}} h_{E}\left\|\mathbf{J}_{E}\left(\left[\mathbf{u}_{h}, p_{h}\right]\right)-\Pi_{E} \mathbf{J}_{E}\left(\left[\mathbf{u}_{h}, p_{h}\right]\right)\right\|_{L^{r^{\prime}(E)}}^{r^{\prime}}\right)^{\frac{1}{r^{\prime}}} \\
+C_{4}\left|\mathbf{u}-\mathbf{u}_{h}\right|_{(\mathbf{u}, r, \alpha)}^{\min \left\{1, \frac{2}{r^{\prime}}\right\}}+\left\|p-p_{h}\right\|_{\mathrm{Q}} .
\end{array}
$$

Finally we use inequality (5.22) to get

$$
\begin{aligned}
\left(\sum_{E \in \mathcal{E}_{h, \Omega}} h_{E}\left\|\Pi_{E} \mathbf{J}_{E}\left(\left[\mathbf{u}_{h}, p_{h}\right]\right)\right\|_{L^{r^{\prime}(E)}}^{r^{\prime}}\right)^{\frac{1}{r^{\prime}}} \precsim 2 C_{4}\left|\mathbf{u}-\mathbf{u}_{h}\right|_{(\mathbf{u}, r, \alpha)}^{\min \left\{1, \frac{2}{r^{\prime}}\right\}}+\left\|p-p_{h}\right\|_{\mathrm{Q}} \\
+\left(\sum_{T \in \mathcal{T}_{h}}\left\|h_{T}\left(\mathbf{R}_{T}\left(\left[\mathbf{u}_{h}, p_{h}\right]\right)-\Pi_{T} \mathbf{R}_{T}\left(\left[\mathbf{u}_{h}, p_{h}\right]\right)\right)\right\|_{L^{r^{\prime}}(T)}^{r^{\prime}}\right)^{\frac{1}{r^{\prime}}} \\
+\left(\sum_{E \in \mathcal{E}_{h, \Omega}} h_{E}\left\|\mathbf{J}_{E}\left(\left[\mathbf{u}_{h}, p_{h}\right]\right)-\Pi_{E} \mathbf{J}_{E}\left(\left[\mathbf{u}_{h}, p_{h}\right]\right)\right\|_{L^{r^{\prime}}(E)}^{r^{\prime}}\right)^{\frac{1}{r^{\prime}}}
\end{aligned}
$$

and the desired result then follows by arguing in exactly the same way as in the proof of Theorem 16 . 


\section{Numerical results on uniform grids}

In this section we report some numerical results to test the behaviour of our estimates with respect to the meshsize $h$ and the parameter $r$.

Remark 24 For the sake of simplicity, the analysis presented in the previous sections concerned homogeneous Dirichlet boundary conditions. It is straightforward to extend the previous analysis to the case of mixed Dirichlet-Neumann boundary conditions. In fact, let us consider the boundary $\Gamma$ split into a relatively closed Dirichlet section $\Gamma_{D}$ with $\left|\Gamma_{D}\right|>0$ and a relatively open Neumann section $\Gamma_{N}=\Gamma \backslash \Gamma_{D}$. On the Neumann boundary we impose the value of the normal stress $\sigma \cdot \hat{n}=\mathbf{g}$. In this case the pressure is defined uniquely rather than up to a constant value only, and therefore $\|p\|_{\mathrm{Q}}$ is simply $\|p\|_{\mathrm{L}^{r^{\prime}(\Omega)}}$ instead of $\inf _{c \in \mathbb{R}}\|p+c\|_{L^{r^{\prime}(\Omega)}}$. In the previous a posteriori error estimates expressed in terms of computable residuals we need to add terms on the boundary faces $E \subset \Gamma_{N}$ similar to the jump terms defined on the internal faces. This can be achieved very simply by adding to the internal face-jumps the quantities $\mathbf{J}_{E}\left(\left[\mathbf{u}_{h}, p_{h}\right]\right)=|\sigma \cdot \hat{n}-\mathbf{g}|_{E}$ defined on the Neumann boundary faces and the corresponding approximations $\Pi_{E} \mathbf{J}_{E}\left(\left[\mathbf{u}_{h}, p_{h}\right]\right)$. In the following, we introduce the notation $\mathcal{E}_{h, \Omega, \Gamma_{N}}=\mathcal{E}_{h, \Omega} \cup \mathcal{E}_{h, N}$ where $\mathcal{E}_{h, N}=\left\{E \in \mathcal{E}_{h}\right.$ : $\left.E \in \Gamma_{N}\right\}$, so as to amalgamate the terms arising on Neumann boundary faces with the terms which appear on internal faces.

In the tables corresponding to the different test problems we report the following computable residuals and jumps involved in our error bounds:

$$
\begin{aligned}
\text { R.res } & =\sum_{T \in \mathcal{T}_{h}} h_{T}^{r^{\prime}}\left\|\Pi_{T} \mathbf{R}_{T}\left(\left[\mathbf{u}_{h}, p_{h}\right]\right)\right\|_{L^{r^{\prime}(T)}}^{r^{\prime}}, \\
\text { R.jumps } & =\sum_{E \in \mathcal{E}_{h, \Omega, \Gamma_{N}}} h_{E}\left\|\Pi_{E} \mathbf{J}_{E}\left(\left[\mathbf{u}_{h}, p_{h}\right]\right)\right\|_{L^{r^{\prime}}(E)}^{r^{\prime}}, \\
\text { R.cont } & =\left\|\nabla \cdot \mathbf{u}_{h}\right\|_{L^{r}(\Omega)}^{r},
\end{aligned}
$$

the errors of our approximate solutions:

$$
\begin{aligned}
\mathrm{E} . \mathrm{Vh} & =\left\|\mathbf{u}-\mathbf{u}_{h}\right\|_{\mathrm{V}}=\left\|e\left(\mathbf{u}-\mathbf{u}_{h}\right)\right\|_{\mathrm{L}^{r}(\Omega)}^{r}, \\
\text { E.ph } & =\left\|p-p_{h}\right\|_{\mathrm{Q}}^{r^{\prime}} .
\end{aligned}
$$

and the two effectivity indices corresponding to the upper and the lower bounds:

$$
\begin{array}{r}
\text { e.i.U }=\left[\left(\sum_{T \in \mathcal{T}_{h}} h_{T}^{r^{\prime}}\left\|\Pi_{T} \mathbf{R}_{T}\left(\left[\mathbf{u}_{h}, p_{h}\right]\right)\right\|_{L^{r^{\prime}}(T)}^{r^{\prime}}\right)^{\frac{\mathrm{R}_{\mathrm{U}}^{\prime}}{r^{\prime}}}+\left(\sum_{E \in \mathcal{E}_{h, \Omega, \Gamma_{N}}} h_{E}\left\|\Pi_{E} \mathbf{J}_{E}\left(\left[\mathbf{u}_{h}, p_{h}\right]\right)\right\|_{L^{r^{\prime}}(E)}^{r^{\prime}}\right)^{\frac{\mathrm{R}_{\mathrm{U}}^{\prime}}{r^{\prime}}}\right. \\
\left.+\left\|\nabla \cdot \mathbf{u}_{h}\right\|_{L^{r}(\Omega)}^{\mathrm{g}^{\prime}}\right]^{\frac{1}{2}} /\left[\left\|\mathbf{u}-\mathbf{u}_{h}\right\|_{\mathrm{V}}^{\mathrm{R}_{\mathrm{U}}}+\left\|p-p_{h}\right\|_{\mathrm{Q}}^{\mathrm{g}}\right]^{\frac{1}{2}},
\end{array}
$$




$$
\begin{array}{r}
\text { e.i.L }=\left[\left(\sum_{T \in \mathcal{T}_{h}} h_{T}^{r^{\prime}}\left\|\Pi_{T} \mathbf{R}_{T}\left(\left[\mathbf{u}_{h}, p_{h}\right]\right)\right\|_{L^{r^{\prime}(T)}}^{r^{\prime}}\right)^{\frac{\mathrm{R}_{\mathrm{L}}^{\prime}}{r^{\prime}}}+\left(\sum_{E \in \mathcal{E}_{h, \Omega, \Gamma_{N}}} h_{E}\left\|\Pi_{E} \mathbf{J}_{E}\left(\left[\mathbf{u}_{h}, p_{h}\right]\right)\right\|_{L^{r^{\prime}(E)}}^{r^{\prime}}\right)^{\frac{\mathrm{R}_{\mathrm{L}}^{\prime}}{r^{\prime}}}\right. \\
\left.+\left\|\nabla \cdot \mathbf{u}_{h}\right\|_{\mathrm{L}^{r}(\Omega)}^{\mathrm{g}^{\prime}}\right]^{\frac{1}{2}} /\left[\left\|\mathbf{u}-\mathbf{u}_{h}\right\|_{\mathrm{V}}^{\mathrm{R}_{\mathrm{L}}}+\left\|p-p_{h}\right\|_{\mathrm{Q}}^{\mathrm{g}}\right]^{\frac{1}{2}} .
\end{array}
$$

All of our numerical solutions are obtained using quadratic finite elements for the velocity components and linear finite elements for the pressure. All the integrals involved in the computation of the residuals and of the true errors are computed with quadrature formulas exact for polynomials of degree seven, so we assume that the approximations of the residuals can be neglected with respect to the other terms. The systems of nonlinear equations are solved by Newton's method supplemented by a line-search globalization method. The Jacobi matrix is preconditioned by the ILUT incomplete LU factorization with drop-tolerance. Failure is declared if the residual of the nonlinear system is greater than 1.0E-10 after 200 iterations. The code is based on the libMesh [17] and PETSc [2] libraries.

\subsection{Problem 1: cavity flow}

The first numerical results we report are obtained on a cavity-like problem in which the flow exhibits a counterclockwise vortex whose centre moves toward the right-vertical wall when increasing $r$ or $\theta$ in the experiments performed on the power-law and the Carreau law, respectively.

For these test problems the domain $\Omega$ is the unit square, we impose the exact value of the normal stress on left-vertical wall and homogeneous Dirichlet boundary conditions for the velocity on the other parts of the boundary of the domain. The exact solution is given by

$$
\begin{aligned}
& u(x, y)=\left(1-\cos \left(2 \frac{\pi\left(e^{\alpha x}-1\right)}{e^{\alpha}-1}\right)\right) \sin (2 \pi y) \\
& v(x, y)=-\alpha e^{\alpha x} \sin \left(2 \frac{\pi\left(e^{\alpha x}-1\right)}{e^{\alpha}-1}\right) \frac{1-\cos (2 \pi y)}{e^{\alpha}-1} \\
& p(x, y)=2 \pi \alpha e^{\alpha x} \sin \left(2 \frac{\pi\left(e^{\alpha x}-1\right)}{e^{\alpha}-1}\right) \frac{\sin (2 \pi y)}{e^{\alpha}-1}
\end{aligned}
$$

where the parameter $\alpha$ has to be replaced by $r$ in the experiments for the power-law and by $\theta$ in the experiments for the Carreau law. The centre of the vortex is located at the point $x_{C}=(1 / \alpha) \log \left(\frac{\mathrm{e}^{\alpha}+1}{2}\right), y_{C}=1 / 2$. The two components of the forcing function are chosen so that the reported expressions for $u, v$ and $p$ are the exact solution when the function $k(\cdot)$ corresponds to the power-law or to the Carreau law, respectively.

The strong nonlinearity imposed a limitation on the range of values of the parameters $r, \mu, \lambda$ for which convergence of the nonlinear problem could be observed on all four grids in our refinement sequence. 
Table 1: Problem 1, power-law: $r=1.8$

\begin{tabular}{|c|c|c|c|c|c|c|c|}
\hline dofs & R.res & R.jump & R.cont & E.Vh & E.ph & e.i.U & e.i.L \\
\hline 295 & 85.55 & 2.428 & $4.555 \mathrm{E}+5$ & $5.262 \mathrm{E}+5$ & 1.06 & 0.4476 & 0.9305 \\
\hline 1091 & 4.812 & 0.07042 & 9114 & $1.11 \mathrm{E}+4$ & 0.04197 & 0.5403 & 0.9065 \\
\hline 4195 & 0.2334 & 0.001307 & 151.7 & 189.2 & 0.001678 & 0.6698 & 0.8962 \\
\hline 16451 & 0.01061 & $2.214 \mathrm{E}-5$ & 3.592 & 4.472 & $7.108 \mathrm{E}-5$ & 0.8266 & 0.8975 \\
\hline
\end{tabular}

Table 2: Problem 1, power-law: $r=2.0$

\begin{tabular}{|c|c|c|c|c|c|c|c|}
\hline dofs & R.res & R.jump & R.cont & E.Vh & E.ph & e.i.U & e.i.L \\
\hline 295 & 57.42 & 2.985 & $1.269 \mathrm{E}+5$ & $1.338 \mathrm{E}+5$ & 1.145 & 0.9739 & 0.9739 \\
\hline 1091 & 4.444 & 0.1383 & 3887 & 4269 & 0.06381 & 0.9548 & 0.9548 \\
\hline 4195 & 0.3003 & 0.003729 & 90.54 & 102.7 & 0.003573 & 0.9406 & 0.9406 \\
\hline 16451 & 0.01925 & $9.059 \mathrm{E}-5$ & 2.148 & 2.425 & $2.139 \mathrm{E}-4$ & 0.9454 & 0.9454 \\
\hline
\end{tabular}

We start by considering the power-law problem, where $k(t)=2 \mu t^{r-2}$. The computations are performed on quasi-uniform triangulations with 70, 294, 1190 and 4774 elements obtained by successive uniform refinement, and with $\mu=1.0 E-3$. From the last two columns of Tables 1-3 we can clearly see the mismatch between the upper and the lower bounds when $r \neq 2$ and that e.i.L is weakly dependent on the meshsize while e.i.U displays a non-negligible dependence on the meshsize.

Tables 4, 5 report the results obtained on the same grid in the case of the Carreau law, $k(t)=k_{\infty}+\left(k_{0}-k_{\infty}\right)\left(1+\lambda t^{2}\right)^{(\theta-2) / 2}$, with $k_{\infty}=1, k_{0}=2, \lambda=1.0 E-5$, the choice of this small value of $\lambda$ being due to the very strong nonlinearity in this test problem; with larger values of $\lambda$ we were unable to achieve convergence of the Newton iteration on all four uniform meshes considered. Since $k_{\infty} \neq 0$, we have $r=2$, so e.i.U=e.i.L for all values of $\theta$. As expected, the effectivity indices are essentially independent of the meshsize.

Table 3: Problem 1, power-law: $r=3.0$

\begin{tabular}{|c|c|c|c|c|c|c|c|}
\hline dofs & R.res & R.jump & R.cont & E.Vh & E.ph & e.i.U & e.i.L \\
\hline 295 & 38.44 & 6.68 & $1.009 \mathrm{E}+4$ & 7136 & 1.53 & 0.2678 & 1.279 \\
\hline 1091 & 5.244 & 0.8402 & 720.6 & 577.4 & 0.231 & 0.3869 & 1.14 \\
\hline 4195 & 0.704 & 0.0516 & 4.793 & 4.219 & 0.02315 & 0.9228 & 1.154 \\
\hline 16451 & 0.09149 & 0.003797 & 0.08016 & 0.07207 & 0.002735 & 1.97 & 1.145 \\
\hline
\end{tabular}


Table 4: Problem 1, Carreau law: $\theta=1.2$

\begin{tabular}{|c|c|c|c|c|c|c|c|}
\hline dofs & R.res & R.jump & R.cont & E.Vh & E.ph & e.i.U & e.i.L \\
\hline 295 & 147.1 & 40.43 & 0.4206 & 0.9132 & 0.559 & 11.3 & 11.3 \\
\hline 1091 & 11.55 & 2.462 & 0.02627 & 0.06653 & 0.02635 & 12.29 & 12.29 \\
\hline 4195 & 0.7757 & 0.1445 & 0.001715 & 0.004457 & 0.00136 & 12.59 & 12.59 \\
\hline 16451 & 0.0522 & 0.008775 & 0.0001095 & 0.0002839 & $7.863 \mathrm{E}-5$ & 12.98 & 12.98 \\
\hline
\end{tabular}

Table 5: Problem 1, Carreau law: $\theta=2.0$

\begin{tabular}{|c|c|c|c|c|c|c|c|}
\hline dofs & R.res & R.jump & R.cont & E.Vh & E.ph & e.i.U & e.i.L \\
\hline 295 & 466.5 & 140 & 1.487 & 3.662 & 1.551 & 10.8 & 10.8 \\
\hline 1091 & 36.31 & 9.31 & 0.1019 & 0.2314 & 0.07375 & 12.24 & 12.24 \\
\hline 4195 & 2.721 & 0.5778 & 0.006635 & 0.01731 & 0.003741 & 12.53 & 12.53 \\
\hline 16451 & 0.1771 & 0.03433 & 0.0004272 & 0.001147 & 0.0002166 & 12.47 & 12.47 \\
\hline
\end{tabular}

\section{Numerical results on adapted grids}

In this section we present some results obtained by using the residual-based a posteriori bounds to adapt the meshes. We are mainly interested in the power-law model for which we have a degenerate viscosity law, but we also consider a Carreau law problem with $k_{\infty}=0$, as it is in these two cases that the sharpness of our estimates is most difficult to ensure in practice. Due to the dependence of the effectivity indices on the meshsize observed in some of our numerical experiments on uniform grids, we believe that these estimates should not be used for reliable and accurate control of the true error. Nevertheless, they provide useful information for the purposes of mesh-adaptivity aimed at improving the results of a computation. Moreover, due to the strong singularity introduced by the power-law model with $r<2$, we noticed occasional strong oscillations of the residual $\Pi_{T} \mathbf{R}_{T}\left(\left[\mathbf{u}_{h}, p_{h}\right]\right)$ in the domain. For these reasons we chose a very simple and somewhat crude adaptive algorithm: we compute, separately, for each element $T \in \mathcal{T}_{h}$, the two quantities

$$
\begin{aligned}
\mathrm{R}_{\mathrm{mom}, \mathrm{T}}=h_{T}^{r^{\prime}}\left\|\Pi_{T} \mathbf{R}_{T}\left(\left[\mathbf{u}_{h}, p_{h}\right]\right)\right\|_{L^{r^{\prime}}(T)}^{r^{\prime}}+ & \frac{1}{2} \sum_{E \in \mathcal{E}(T) \cap \mathcal{E}_{h, \Omega}} h_{E}\left\|\Pi_{E} \mathbf{J}_{E}\left(\left[\mathbf{u}_{h}, p_{h}\right]\right)\right\|_{L^{r^{\prime}}(E)}^{r^{\prime}} h_{E}\left\|\Pi_{E} \mathbf{J}_{E}\left(\left[\mathbf{u}_{h}, p_{h}\right]\right)\right\|_{L^{r^{\prime}}(E)}^{r^{\prime}} \\
& +\sum_{E \in \mathcal{E}(T) \cap \mathcal{E}_{h, N}} h
\end{aligned}
$$

and $\mathrm{R}_{\text {cont,T }}=\left\|\nabla \cdot \mathbf{u}_{h}\right\|_{L^{r}(T)}^{r}$. Then we sort each of the two vectors and we split our marking stage into two distinct steps. First we select for refinement a fixed percentage of the elements having the largest values $R_{\text {mom, } T}$; we then do the same for $R_{\text {cont,T }}$ separately. Second, we select for coarsening a fixed percentage of the elements having the smallest 
value $R_{\text {mom,T }}$, respectively $R_{\text {cont,T }}$. We then explicitly mark for refinement the elements which have been selected for refinement according to at least one of their values $R_{\text {mom, }}$ or $R_{\text {cont,T }}$ and we explicitly mark for coarsening the elements which have been selected for coarsening according to both $R_{\text {mom, } T}$ and $R_{\text {cont,T }}$. All the elements marked for refinement are then refined, but the elements marked for coarsening are coarsened only if this operation is compatible with the action we have to perform on the neighbouring elements, i.e. if none of the neighbouring elements have to be refined and if all children of a given parent element are marked for coarsening. To make the adaptive process more flexible we allow the presence of hanging nodes in the refined meshes.

To test the behaviour of our adaptive method we consider a linear problem $[11,26]$ and we apply our adaptive method to it. The problem has the following exact solution in polar coordinates:

$$
\begin{aligned}
u(r, \phi) & =r^{\alpha}\left((1+\alpha) \sin (\phi) w(\phi)+\cos (\phi) w_{\phi}(\phi)\right), \\
v(r, \phi) & =r^{\alpha}\left(-(1+\alpha) \cos (\phi) w(\phi)+\sin (\phi) w_{\phi}(\phi)\right), \\
p(r, \phi) & =-r^{\alpha-1} \frac{(1+\alpha)^{2} w_{\phi}(\phi)+w_{\phi \phi \phi}(\phi)}{1-\alpha}, \\
w(\phi) & =\frac{\sin ((1+\alpha) \phi) \cos (\alpha \omega)}{1+\alpha}-\cos ((1+\alpha) \phi)-\frac{\sin ((1-\alpha) \phi) \cos (\alpha \omega)}{1-\alpha}+\cos ((1-\alpha) \phi),
\end{aligned}
$$

where $\alpha=856399 / 1572864$ and $\omega=3 \pi / 2$. The forcing function $f$ is identically zero, the viscosity coefficient is $\mu=1$ and the computational domain $\Omega$ is the L-shaped domain $\Omega=(-1,1)^{2} \backslash[0,1] \times[-1,0]$.

In the linear case $(r=2)$ our estimates collapse to the classical estimates for the Stokes problem by Verfürth. In Table 6 we report the results obtained by performing seven adaptive steps. We start by marking for refinement $40 \%$ of the elements having the largest values $R_{\text {mom,T }}$ and $R_{\text {cont,T }}$ and by marking for coarsening $4 \%$ of the elements having the smallest values $R_{\text {mom, } T}$ and $R_{\text {cont, } T}$. In each adaptive iteration we divide by two these percentages. These are the values that we shall use in all of our adaptive test problems. The reduction of the refinement percentage of the elements is motivated by the fact that increasing the total number of the active elements and keeping this percentage fixed would result in adding more and more elements in each iteration and this makes the solution of the resulting nonlinear systems increasingly more difficult. In Figures 4, 5 we report the initial and the final mesh. This adaptive method can lead to a small over-refinement due to the fact that it deals separately with the momentum residual and the continuity residual even when one of these is much smaller than the other. We postpone to a future study the development of an improved marking strategy; for the moment, this appears to be the simplest approach for dealing with the different powers of the two residuals in the upper and lower bounds. The nonoptimality of the marking strategy is probably the cause of the moderate increase of the effectivity index we observe in Table 6 under mesh refinement. Nevertheless, the resulting final mesh in Figure 5 displays a clear refinement around the re-entrant corner and is quite clean away from it.

The geometries that we consider for the remaining adaptive tests are two channels 
Table 6: Linear adaptive test problem: error estimators and true errors during the adaptive process

\begin{tabular}{|c|c|c|c|c|c|c|c|}
\hline dofs & R.res & R.jump & R.cont & E.Vh & E.ph & e.i.U & e.i.L \\
\hline 259 & 18.34 & 12.48 & 0.6418 & 1.045 & 3.575 & 2.609 & 2.609 \\
\hline 654 & 8.689 & 5.851 & 0.2977 & 0.4892 & 1.688 & 2.611 & 2.611 \\
\hline 1240 & 4.109 & 2.741 & 0.1387 & 0.2287 & 0.7926 & 2.616 & 2.616 \\
\hline 1835 & 2.017 & 1.309 & 0.0653 & 0.1079 & 0.3732 & 2.655 & 2.655 \\
\hline 2232 & 1.057 & 0.6438 & 0.03108 & 0.05149 & 0.1765 & 2.756 & 2.756 \\
\hline 2482 & 0.6188 & 0.3375 & 0.01509 & 0.02511 & 0.08412 & 2.982 & 2.982 \\
\hline 2629 & 0.4326 & 0.2018 & 0.007636 & 0.01286 & 0.04094 & 3.454 & 3.454 \\
\hline
\end{tabular}
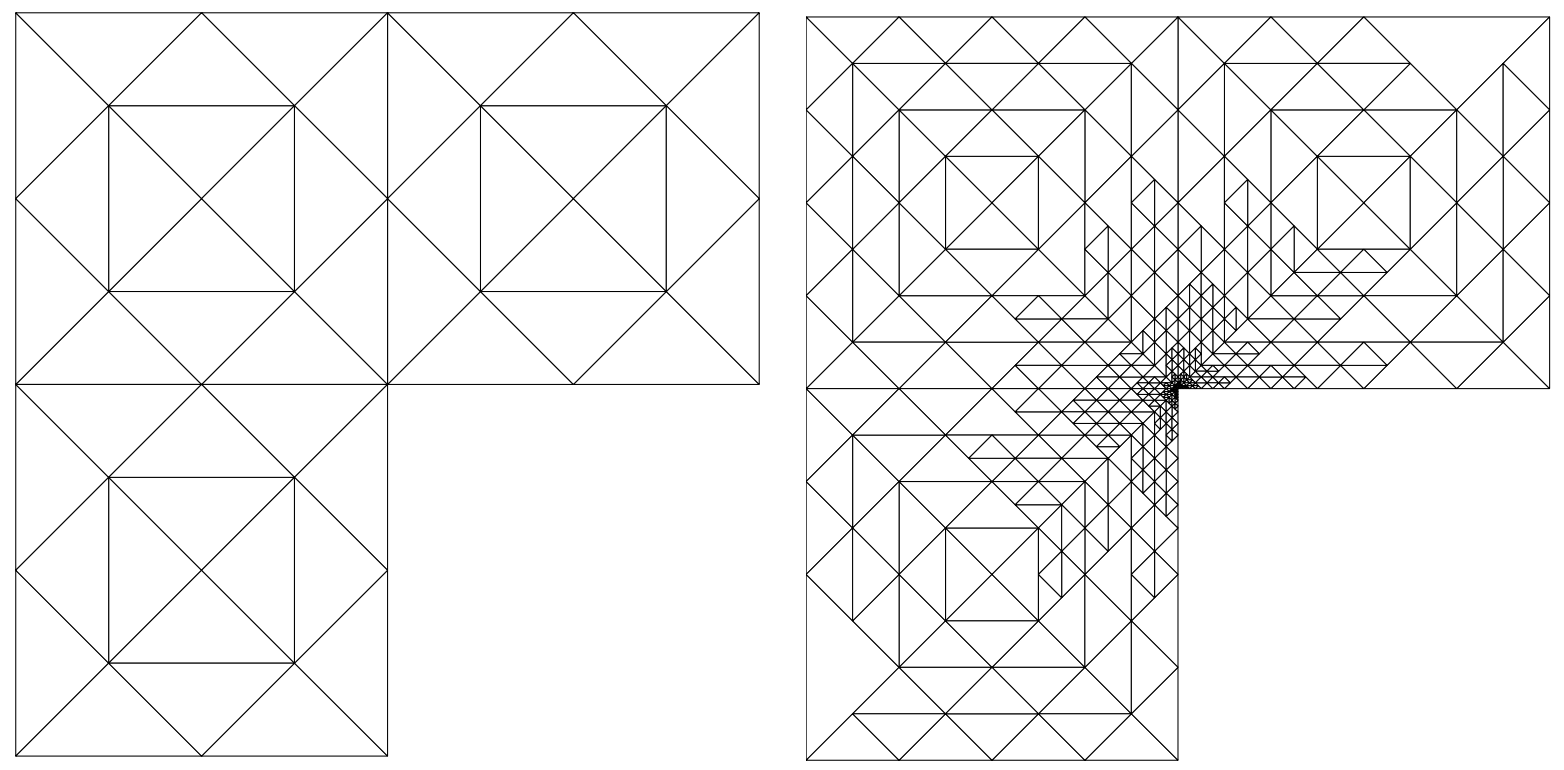

Figure 4: Linear adaptive test problem: Figure 5: Linear adaptive test problem, fistarting mesh nal mesh 


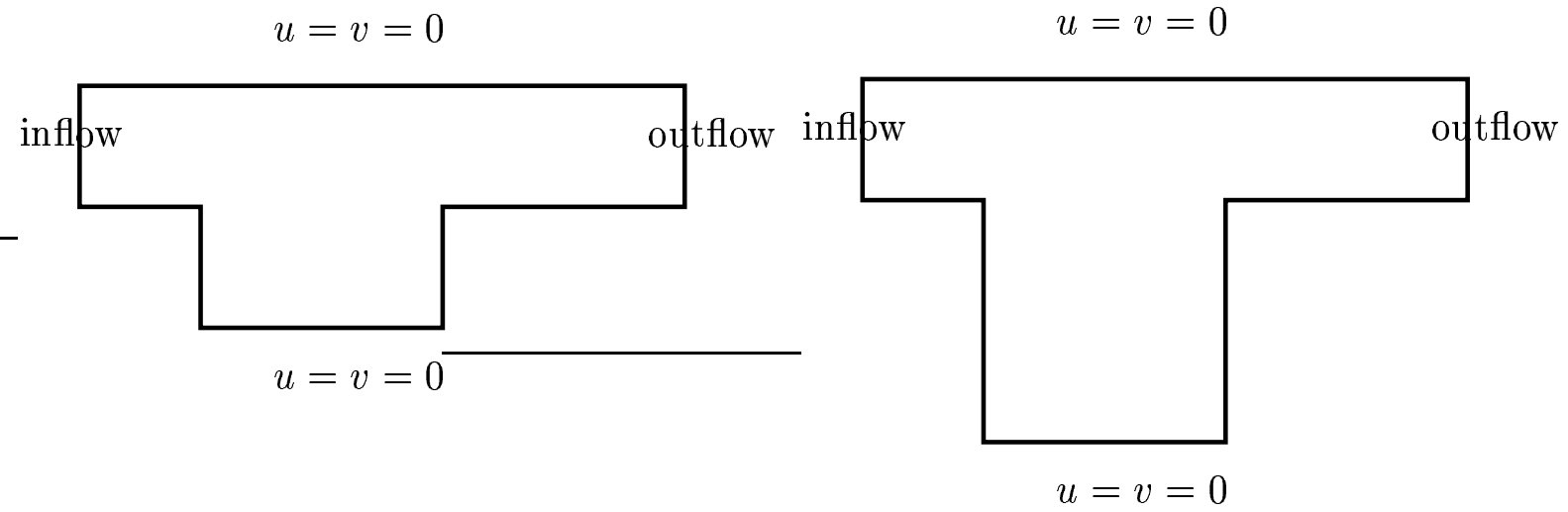

Figure 6: Geometry of the domain $\Omega_{1} \quad$ Figure 7: Geometry of the domain $\Omega_{2}$

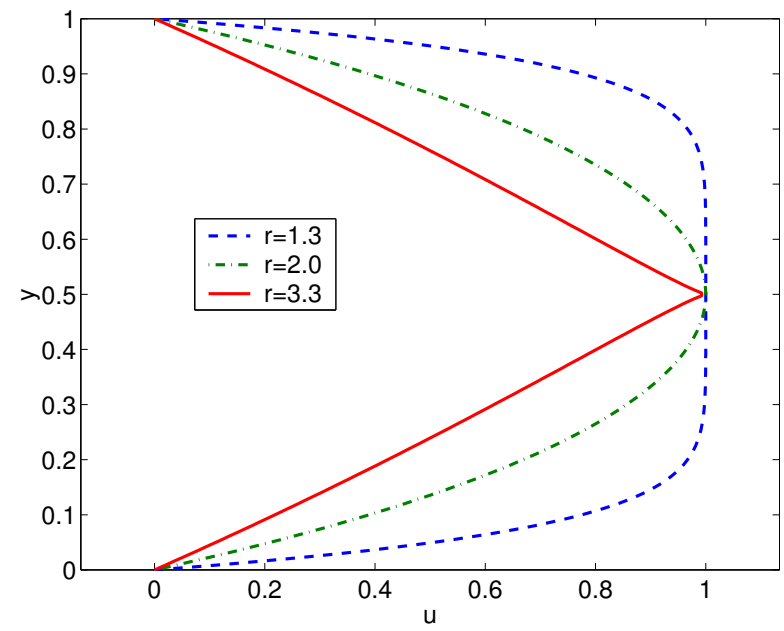

Figure 8: Exact velocity profiles for fully developed power-law flows

of unit height and five units long each, with a cavity in the lower wall which is either one or two units deep (called domains $\Omega_{1}$ and $\Omega_{2}$ respectively); see Figures 6 and 7 .

\subsection{Adaptive problem 1: power-law}

In this test problem we impose the exact velocity profile for the fully developed power-law flow (Figure 8) at the inflow and the exact values of the normal stress for a fully developed flow at the outflow, and we consider two values of $r$, one giving a singular viscosity coefficient $(r=1.3)$ and the other giving a possibly degenerate viscosity coefficient $(r=3.3)$.

\subsubsection{Singular case $r=1.3$}

The computations are performed with the following parameters for the power-law model: $r=1.3, \mu=1.0 E-2$. 
Table 7: Adaptive problem 1, power-law, $r=1.3, \Omega_{1}$ : error estimators during the adaptive process

\begin{tabular}{|c|c|c|c|c|c|}
\hline dofs & R.res & R.jump & R.cont & tot.R.U & tot.R.L \\
\hline 635 & $2.7314 \mathrm{E}-03$ & $6.7143 \mathrm{E}-08$ & $4.5333 \mathrm{E}-01$ & $5.1941 \mathrm{E}-01$ & $4.5606 \mathrm{E}-01$ \\
\hline 1479 & $4.5745 \mathrm{E}-05$ & $2.4162 \mathrm{E}-08$ & $2.3615 \mathrm{E}-01$ & $2.4639 \mathrm{E}-01$ & $2.3620 \mathrm{E}-01$ \\
\hline 3294 & $3.9160 \mathrm{E}-05$ & $7.7523 \mathrm{E}-09$ & $1.1584 \mathrm{E}-01$ & $1.2527 \mathrm{E}-01$ & $1.1588 \mathrm{E}-01$ \\
\hline 5645 & $3.4372 \mathrm{E}-06$ & $2.8258 \mathrm{E}-09$ & $5.7967 \mathrm{E}-02$ & $6.1088 \mathrm{E}-02$ & $5.7970 \mathrm{E}-02$ \\
\hline 7836 & $8.7147 \mathrm{E}-07$ & $1.3763 \mathrm{E}-09$ & $3.5581 \mathrm{E}-02$ & $3.7259 \mathrm{E}-02$ & $3.5582 \mathrm{E}-02$ \\
\hline 9437 & $6.5104 \mathrm{E}-07$ & $7.0604 \mathrm{E}-10$ & $2.4311 \mathrm{E}-02$ & $2.5766 \mathrm{E}-02$ & $2.4312 \mathrm{E}-02$ \\
\hline 10339 & $6.6626 \mathrm{E}-07$ & $3.7340 \mathrm{E}-10$ & $1.9093 \mathrm{E}-02$ & $2.0548 \mathrm{E}-02$ & $1.9093 \mathrm{E}-02$ \\
\hline
\end{tabular}

Table 8: Adaptive problem 1, power-law, $r=1.3, \Omega_{2}$ : error estimators during the adaptive process

\begin{tabular}{|c|c|c|c|c|c|}
\hline dofs & R.res & R.jump & R.cont & tot.R.U & tot.R.L \\
\hline 753 & $2.7953 \mathrm{E}-03$ & $1.0252 \mathrm{E}-07$ & $4.5013 \mathrm{E}-01$ & $5.1248 \mathrm{E}-01$ & $4.5292 \mathrm{E}-01$ \\
\hline 1961 & $4.7504 \mathrm{E}-04$ & $3.7595 \mathrm{E}-08$ & $2.1887 \mathrm{E}-01$ & $2.4592 \mathrm{E}-01$ & $2.1935 \mathrm{E}-01$ \\
\hline 4384 & $5.1211 \mathrm{E}-05$ & $1.0806 \mathrm{E}-08$ & $1.0146 \mathrm{E}-01$ & $1.1095 \mathrm{E}-01$ & $1.0152 \mathrm{E}-01$ \\
\hline 7478 & $4.2915 \mathrm{E}-06$ & $4.2163 \mathrm{E}-09$ & $5.2594 \mathrm{E}-02$ & $5.5584 \mathrm{E}-02$ & $5.2598 \mathrm{E}-02$ \\
\hline 10532 & $9.0763 \mathrm{E}-07$ & $2.0577 \mathrm{E}-09$ & $2.9338 \mathrm{E}-02$ & $3.0797 \mathrm{E}-02$ & $2.9339 \mathrm{E}-02$ \\
\hline 12766 & $2.2048 \mathrm{E}-06$ & $1.0515 \mathrm{E}-09$ & $1.8854 \mathrm{E}-02$ & $2.1013 \mathrm{E}-02$ & $1.8856 \mathrm{E}-02$ \\
\hline 14082 & $5.1441 \mathrm{E}-07$ & $5.4362 \mathrm{E}-10$ & $1.4126 \mathrm{E}-02$ & $1.5223 \mathrm{E}-02$ & $1.4126 \mathrm{E}-02$ \\
\hline
\end{tabular}

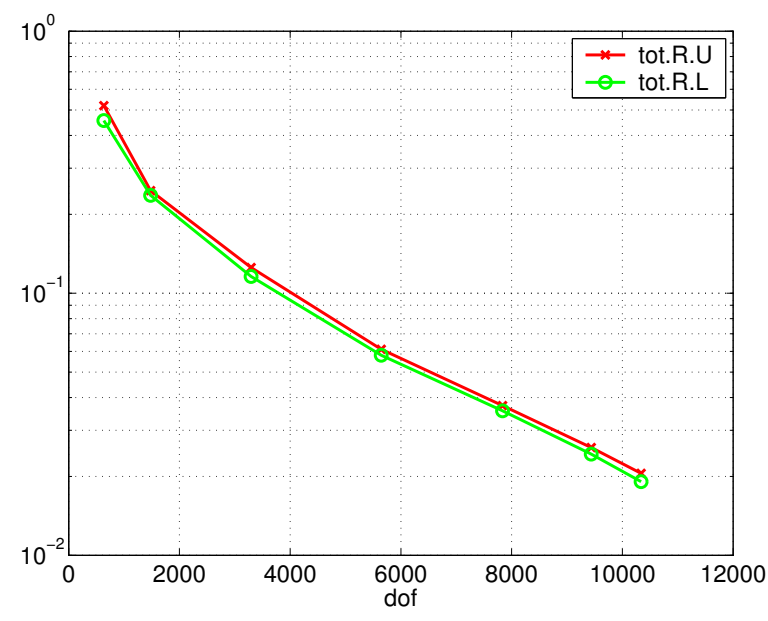
Figure 9: Adaptive problem 1 , power-law,
$r=1.3$ : total residuals during the adaptive process on $\Omega_{1}$

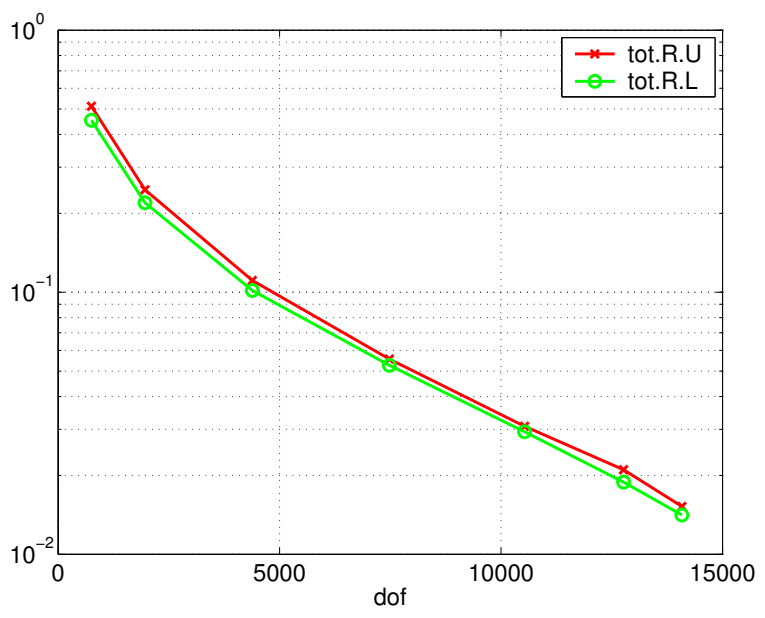

Figure 10: Adaptive problem 1, power-law, $r=1.3$ : total residuals during the adaptive process on $\Omega_{2}$ 

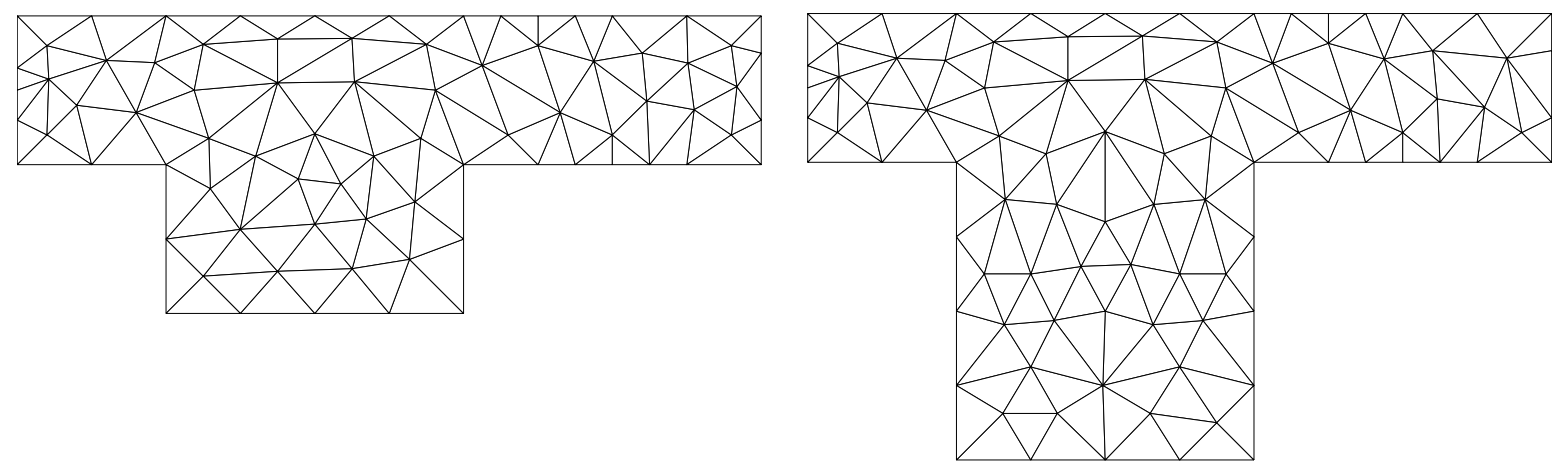

Figure 11: Adaptive problem 1, power-law, Figure 12: Adaptive problem 1, power-law, $r=1.3$ : starting mesh on the domain $\Omega_{1} \quad r=1.3$ : starting mesh on the domain $\Omega_{2}$
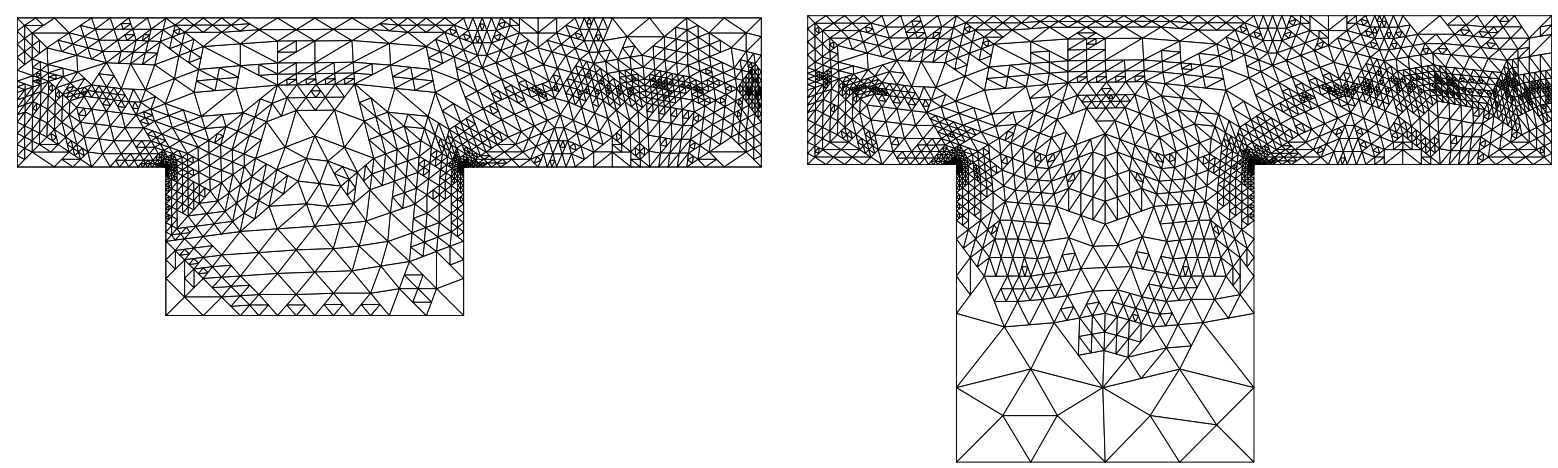

Figure 13: Adaptive problem 1, power-law, Figure 14: Adaptive problem 1, power-law, $r=1.3:$ final mesh on the domain $\Omega_{1}$ $r=1.3$ : final mesh on the domain $\Omega_{2}$
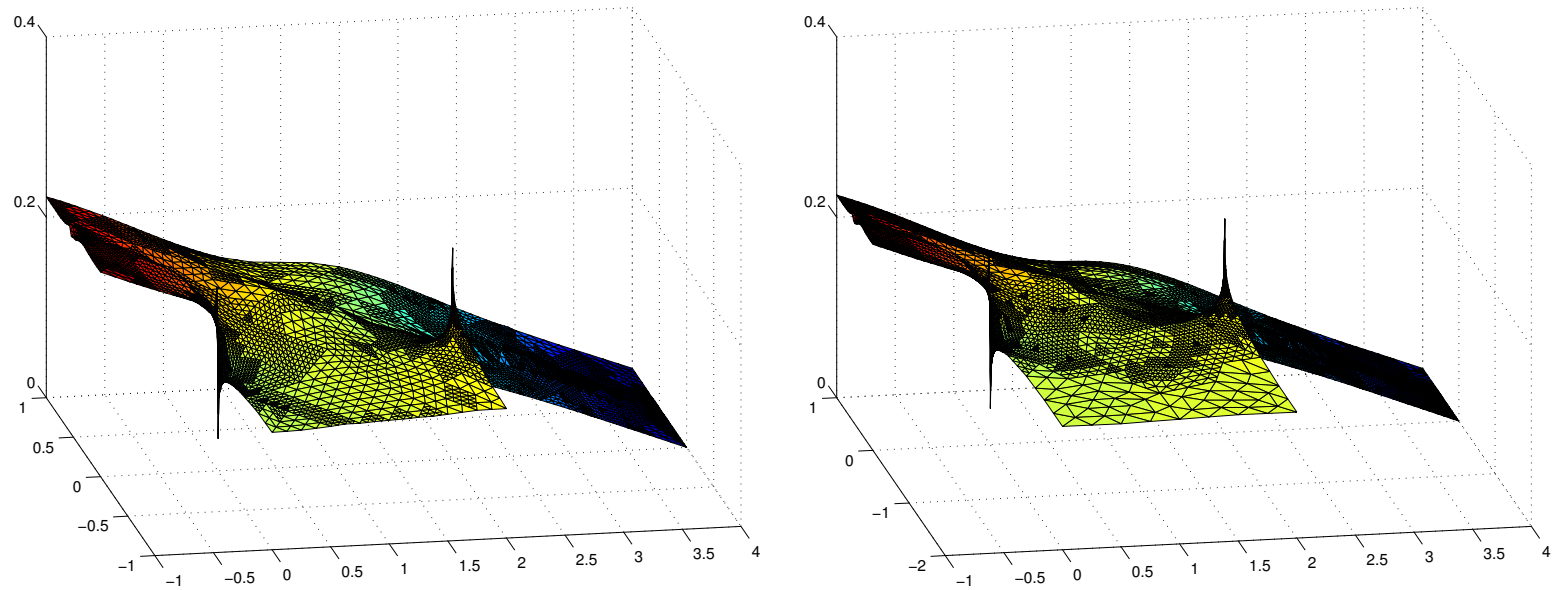

Figure 15: Adaptive problem 1, power-law, Figure 16: Adaptive problem 1, power-law, $r=1.3:$ pressure surface on the final mesh on the domain $\Omega_{1}$

$r=1.3$ : pressure surface on the final mesh on the domain $\Omega_{2}$ 

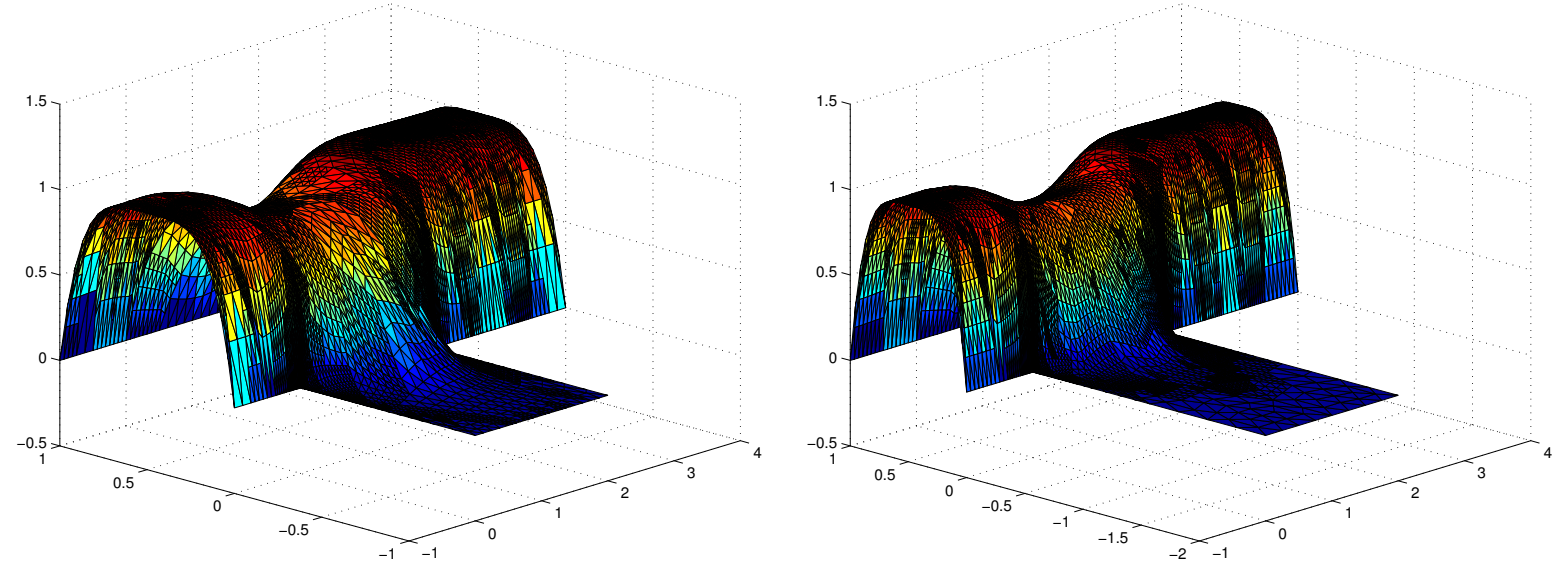

Figure 17: Adaptive problem 1, power-law, Figure 18: Adaptive problem 1, power-law, $r=1.3: u$ velocity component on the final $r=1.3: u$ velocity component on the final mesh on the domain $\Omega_{1}$ mesh on the domain $\Omega_{2}$

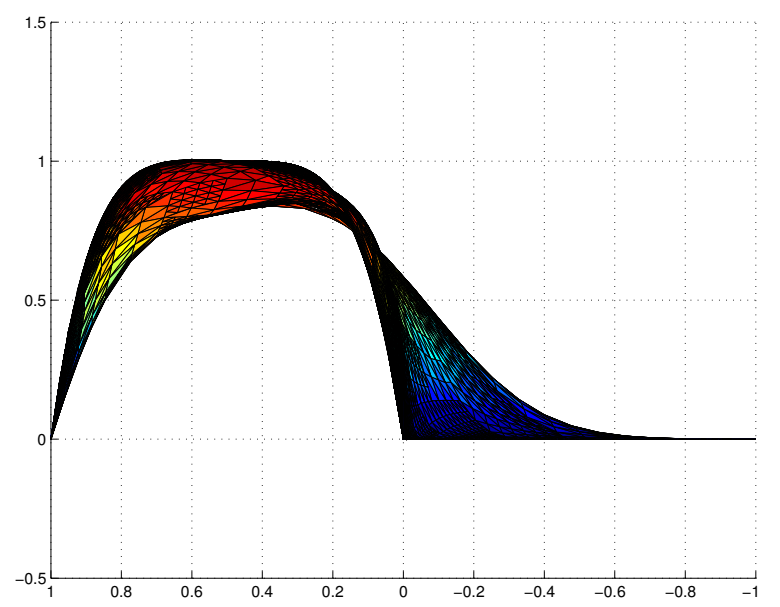

Figure 19: Adaptive problem 1, power-law $r=1.3: u$ velocity component profile on the final mesh on the domain $\Omega_{1}$

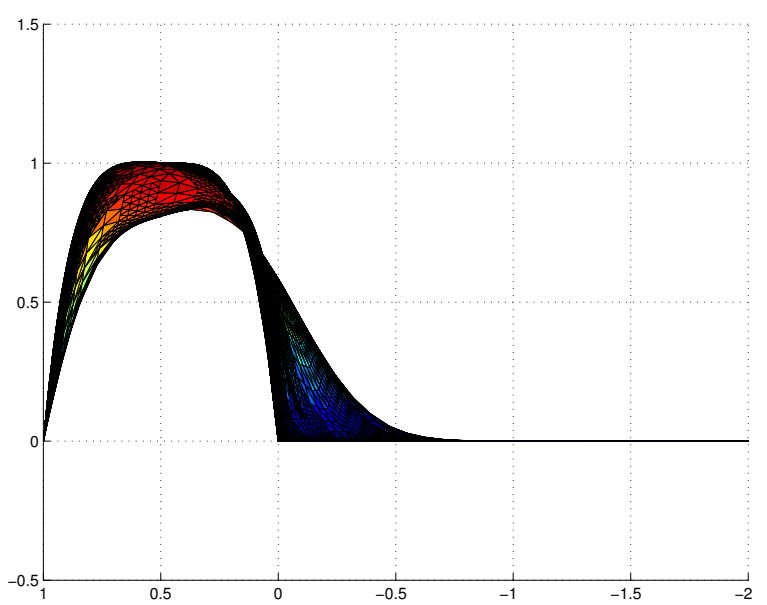

Figure 20: Adaptive problem 1, power-law, $r=1.3: u$ velocity component profile on the final mesh on the domain $\Omega_{2}$ 


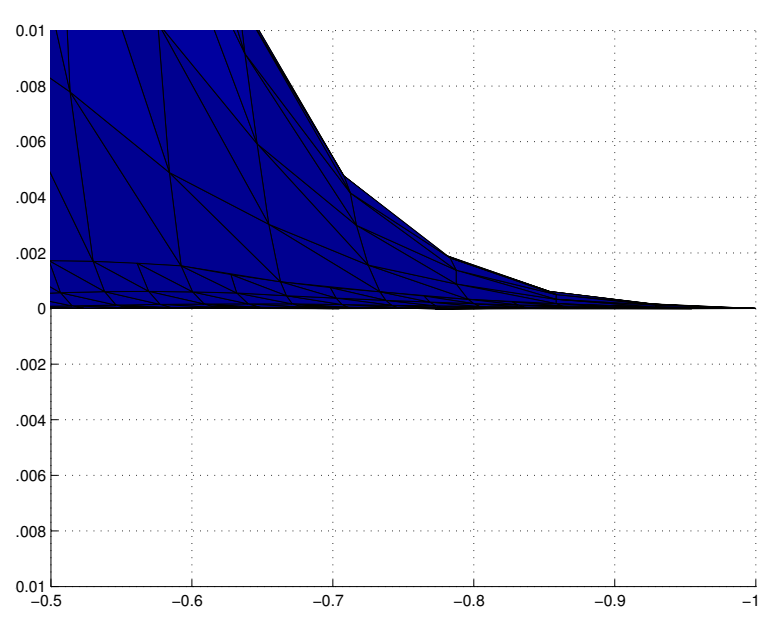

Figure 21: Adaptive problem 1, power-law $r=1.3: u$ velocity component profile on the final mesh on the domain $\Omega_{1}$; detail in the lower part of the cavity

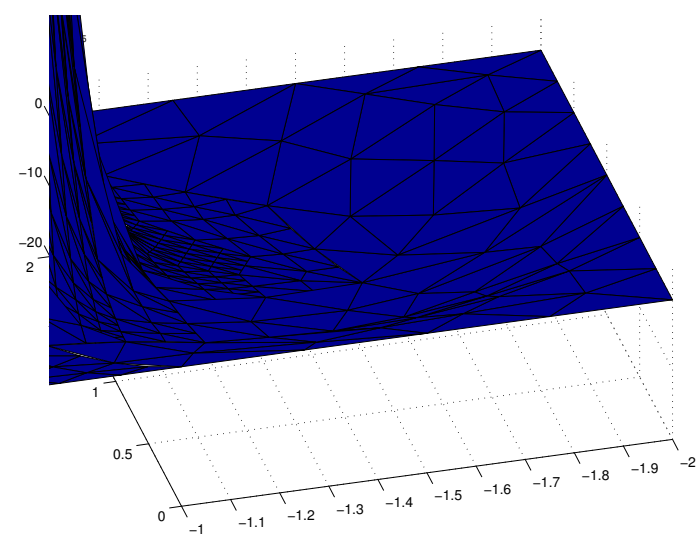

Figure 23: Adaptive problem 1, power-law, $r=1.3: u$ velocity component on the final mesh on the domain $\Omega_{2}$; detail in the lower part of the cavity

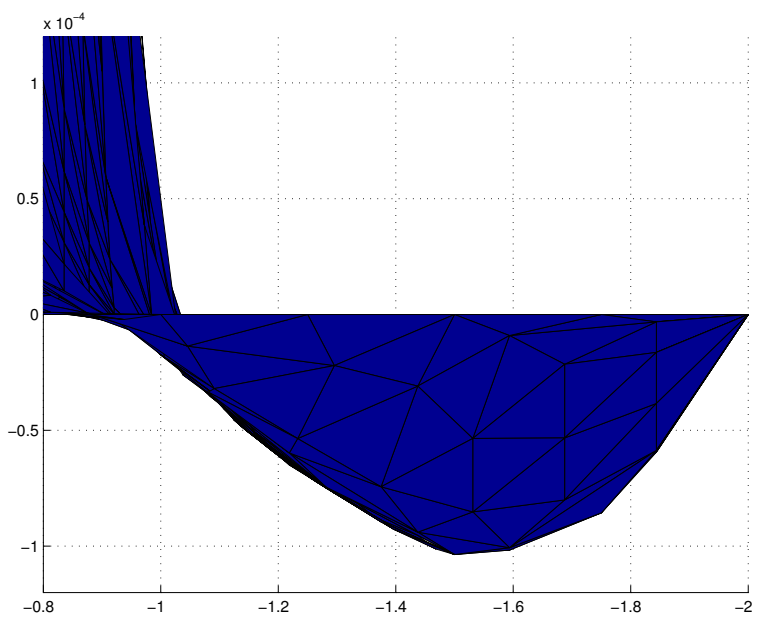

Figure 22: Adaptive problem 1, power-law, $r=1.3: u$ velocity component profile on the final mesh on the domain $\Omega_{2}$; detail in the lower part of the cavity

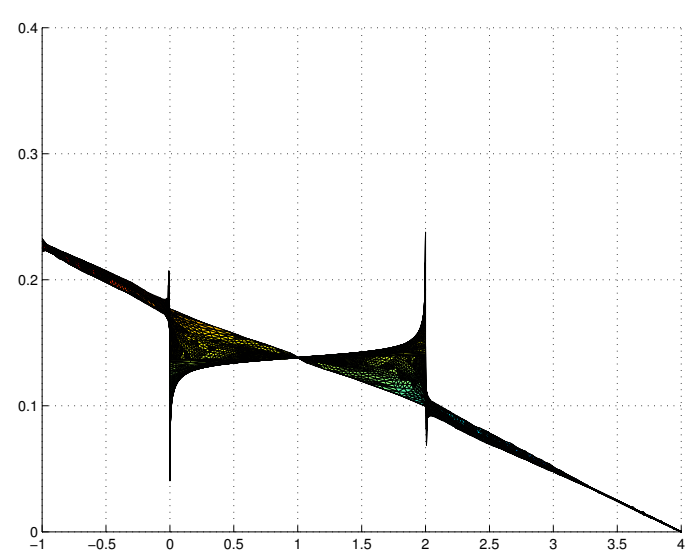

Figure 24: Adaptive problem 1, power-law, $r=1.3$ : pressure profile on the final mesh on the domain $\Omega_{2}$ 
In Table 7 we report the residuals R.res, R.jumps and R.cont in the same way as in the tables of the previous section, and the quantities

$$
\begin{aligned}
\text { tot.R.U }= & \left(\sum_{T \in \mathcal{T}_{h}} h_{T}^{r^{\prime}}\left\|\Pi_{T} \mathbf{R}_{T}\left(\left[\mathbf{u}_{h}, p_{h}\right]\right)\right\|_{L^{r^{\prime}(T)}}^{r^{\prime}}\right)^{\frac{\mathrm{R}_{\mathrm{U}}^{\prime}}{r^{\prime}}} \\
& +\left(\sum_{E \in \mathcal{E}_{h, \Omega, \Gamma_{N}}} h_{E}\left\|\Pi_{E} \mathbf{J}_{E}\left(\left[\mathbf{u}_{h}, p_{h}\right]\right)\right\|_{L^{r^{\prime}}(E)}^{r^{\prime}}\right)^{\frac{\mathrm{R}_{\mathrm{U}}^{\prime}}{r^{\prime}}}+\left\|\nabla \cdot \mathbf{u}_{h}\right\|_{L^{r}(\Omega)}^{g^{\prime}} \\
\text { tot.R.L }= & \left(\sum_{T \in \mathcal{T}_{h}} h_{T}^{r^{\prime}}\left\|\Pi_{T} \mathbf{R}_{T}\left(\left[\mathbf{u}_{h}, p_{h}\right]\right)\right\|_{L^{r^{\prime}}(T)}^{r^{\prime}}\right)^{\frac{\mathrm{R}_{\mathrm{L}}^{\prime}}{r^{\prime}}} \\
& +\left(\sum_{E \in \mathcal{E}_{h, \Omega, \Gamma_{N}}} h_{E}\left\|\Pi_{E} \mathbf{J}_{E}\left(\left[\mathbf{u}_{h}, p_{h}\right]\right)\right\|_{L^{r^{\prime}}(E)}^{r^{\prime}}\right)^{\frac{\mathrm{R}_{\mathrm{I}}^{\prime}}{r^{\prime}}}+\left\|\nabla \cdot \mathbf{u}_{h}\right\|_{L^{r}(\Omega)}^{g^{\prime}}
\end{aligned}
$$

obtained on the domain $\Omega_{1}$, and in Table 8 we report the same quantities obtained on $\Omega_{2}$. The main difference between these two problems is that in the second case the solution exhibits a very slow clockwise vortex inside the cavity. In Figures 9 and 10 we plot the last two columns of the Tables 7 and 8 . We can see that the two total residuals stay very close to each other throughout the adaptive process. In Figures 11 and 13 we show the starting mesh and the final mesh produced by the adaptive algorithm applied on the domain $\Omega_{1}$, while in Figures 12 and 14 we show the starting mesh and the final mesh produced by the adaptive algorithm applied on the domain $\Omega_{2}$.

In the adapted grids of Figures 13 and 14 we have a strong refinement around the two upper corners of the cavity. We can also see mesh refinement along the centrelines of the inflow and of the outflow channels where $\left|\nabla \mathbf{u}_{h}\right|^{r-2} \gg 1$. In other words, refinement along the centre-lines of the inflow and outflow channel appears to be caused by the singularity in the nonlinear viscosity model when the velocity gradient vanishes. In these parts of the domain the flow is essentially the fully developed flow that displays a vanishing gradient in the centre of the channel.

In Figure 14 we can also recognise a refined region inside the cavity containing the center of the vortex that we have in the lower part of the cavity. This refinement is not present in the solution corresponding to the domain $\Omega_{1}$ where this vortex is absent. In Figures 19 and 20 we report the profiles of the $u$ component of the velocity on the two domains. The point of view is from the inflow direction. In Figure 21 we show a zoom into this profile and we can see that on $\Omega_{1}$ the first component of the velocity vanishes towards the bottom of the cavity while remaining nonnegative. In Figure 22 we show a detail of the profile of $u$ in the lower part of the cavity of the domain $\Omega_{2}$ and we see the negative values of $u$ in the lower part of the clockwise vortex at the bottom of the cavity. We can also notice that these values are very small due to the fact that the very small values of the derivatives of the velocity give rise to very large values of the 
Table 9: Adaptive problem 1, power-law, $r=3.3, \Omega_{1}$ : error estimators during the adaptive process

\begin{tabular}{|c|c|c|c|c|c|}
\hline dofs & R.res & R.jump & R.cont & tot.R.U & tot.R.L \\
\hline 635 & $3.9478 \mathrm{E}-02$ & $1.7220 \mathrm{E}-02$ & $5.0579 \mathrm{E}-02$ & $2.2058 \mathrm{E}-01$ & $1.7841 \mathrm{E}-01$ \\
\hline 1396 & $1.3360 \mathrm{E}-02$ & $7.0335 \mathrm{E}-03$ & $2.2530 \mathrm{E}-02$ & $1.2078 \mathrm{E}-01$ & $1.0383 \mathrm{E}-01$ \\
\hline 2692 & $5.8042 \mathrm{E}-03$ & $3.1175 \mathrm{E}-03$ & $9.9591 \mathrm{E}-03$ & $7.0128 \mathrm{E}-02$ & $6.2291 \mathrm{E}-02$ \\
\hline 4548 & $3.0093 \mathrm{E}-03$ & $1.5369 \mathrm{E}-03$ & $4.6169 \mathrm{E}-03$ & $4.2957 \mathrm{E}-02$ & $3.8836 \mathrm{E}-02$ \\
\hline 6217 & $1.8593 \mathrm{E}-03$ & $8.9001 \mathrm{E}-04$ & $2.1729 \mathrm{E}-03$ & $2.7076 \mathrm{E}-02$ & $2.4539 \mathrm{E}-02$ \\
\hline 7780 & $1.3605 \mathrm{E}-03$ & $6.0682 \mathrm{E}-04$ & $1.0273 \mathrm{E}-03$ & $1.7417 \mathrm{E}-02$ & $1.5583 \mathrm{E}-02$ \\
\hline 8597 & $1.1390 \mathrm{E}-03$ & $4.6404 \mathrm{E}-04$ & $4.8882 \mathrm{E}-04$ & $1.1453 \mathrm{E}-02$ & $9.9512 \mathrm{E}-03$ \\
\hline
\end{tabular}

Table 10: Adaptive problem 1, power-law, $r=3.3, \Omega_{2}$ : error estimators during the adaptive process

\begin{tabular}{|c|c|c|c|c|c|}
\hline dofs & R.res & R.jump & R.cont & tot.R.U & tot.R.L \\
\hline 753 & $4.4187 \mathrm{E}-02$ & $1.8418 \mathrm{E}-02$ & $5.1408 \mathrm{E}-02$ & $2.2811 \mathrm{E}-01$ & $1.8225 \mathrm{E}-01$ \\
\hline 1735 & $1.3432 \mathrm{E}-02$ & $7.1411 \mathrm{E}-03$ & $2.3395 \mathrm{E}-02$ & $1.2328 \mathrm{E}-01$ & $1.0618 \mathrm{E}-01$ \\
\hline 3328 & $5.4933 \mathrm{E}-03$ & $3.0068 \mathrm{E}-03$ & $1.0377 \mathrm{E}-02$ & $7.1253 \mathrm{E}-02$ & $6.3765 \mathrm{E}-02$ \\
\hline 5122 & $2.8715 \mathrm{E}-03$ & $1.5320 \mathrm{E}-03$ & $4.8263 \mathrm{E}-03$ & $4.3861 \mathrm{E}-02$ & $3.9863 \mathrm{E}-02$ \\
\hline 7073 & $1.7598 \mathrm{E}-03$ & $8.6224 \mathrm{E}-04$ & $2.2704 \mathrm{E}-03$ & $2.7605 \mathrm{E}-02$ & $2.5181 \mathrm{E}-02$ \\
\hline 8684 & $1.2657 \mathrm{E}-03$ & $5.5847 \mathrm{E}-04$ & $1.0757 \mathrm{E}-03$ & $1.7710 \mathrm{E}-02$ & $1.6007 \mathrm{E}-02$ \\
\hline 9569 & $1.0330 \mathrm{E}-03$ & $4.2030 \mathrm{E}-04$ & $5.1128 \mathrm{E}-04$ & $1.1575 \mathrm{E}-02$ & $1.0210 \mathrm{E}-02$ \\
\hline
\end{tabular}

viscosity coefficient. From Figure 23 we can see that the refined region inside the cavity corresponds to the region where we have strong changes in the curvature of $u$ exactly where the first component of the velocity changes from positive to negative values.

In Figure 24 we report the pressure profile; the point of view is from the bottom of the cavity. We can see that the pressure singularities in the top corners of the cavity are well detected in our adapted solution thanks to the strong refinement of the mesh in these regions.

\subsubsection{Nonsingular case $r=3.3$}

In this case we take $r=3.3$; the geometry, the starting grid as well as all the adaptive parameters are unchanged.

In Tables 9 and 10 we report the residuals and the error estimators as in the previous cases. In Figures 25 and 26 we plot the last two columns of the Tables 9 and 10; again the upper and the lower total residuals are very close to each other. In Figures 27 and 28 we show the final meshes produced by the adaptive algorithm. In Figures 27 and 28 we 


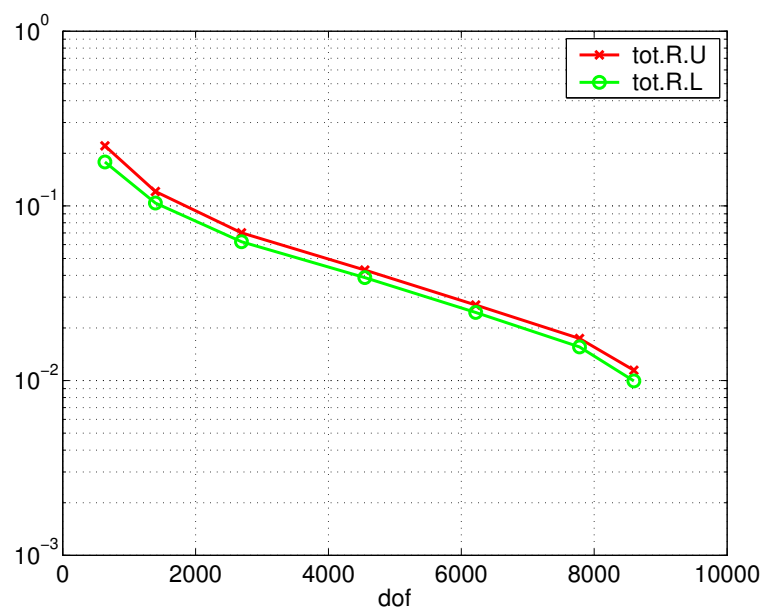
Figure 25: Adaptive problem 1 , power-law,
$r=3.3$ : total residuals during the adaptive process on $\Omega_{1}$
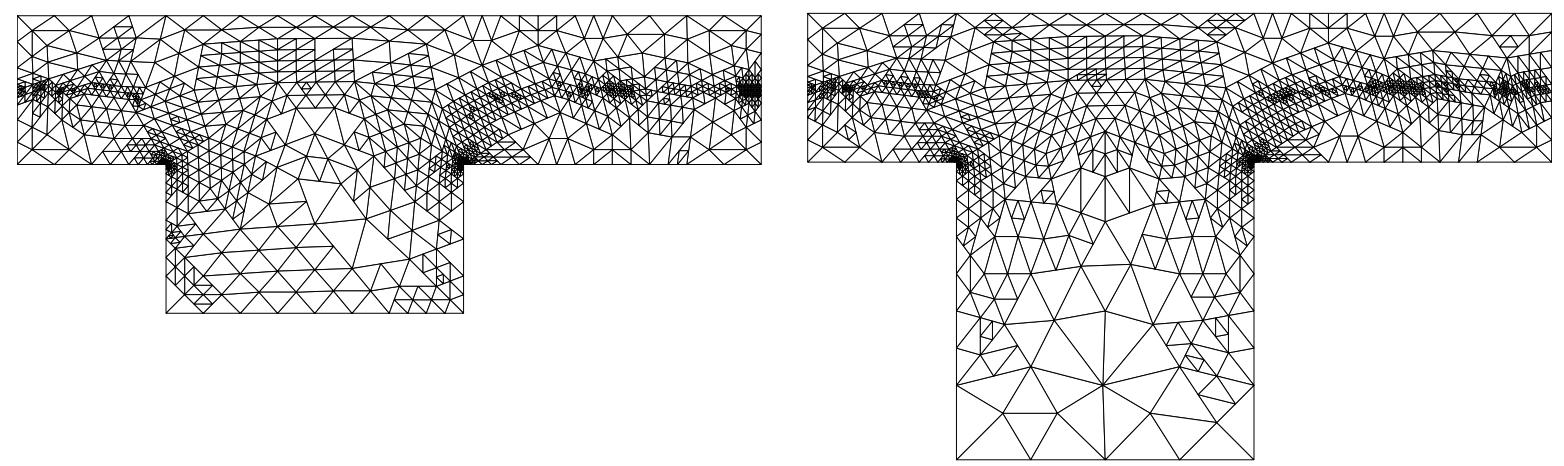

Figure 27: Adaptive problem 1, power-law, Figure 28: Adaptive problem 1, power-law, $r=3.3:$ final mesh on the domain $\Omega_{1}$

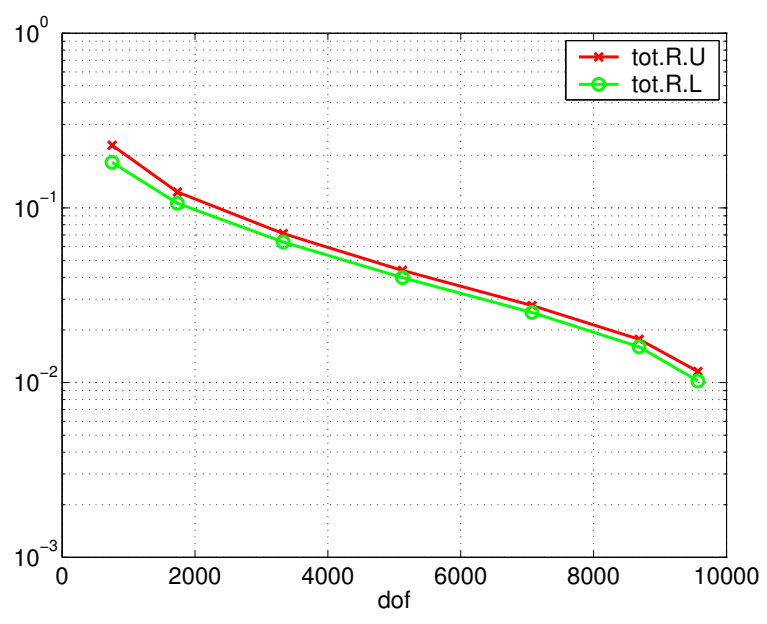

Figure 26: Adaptive problem 1, power-law, $r=3.3$ : total residuals during the adaptive process on $\Omega_{2}$ 


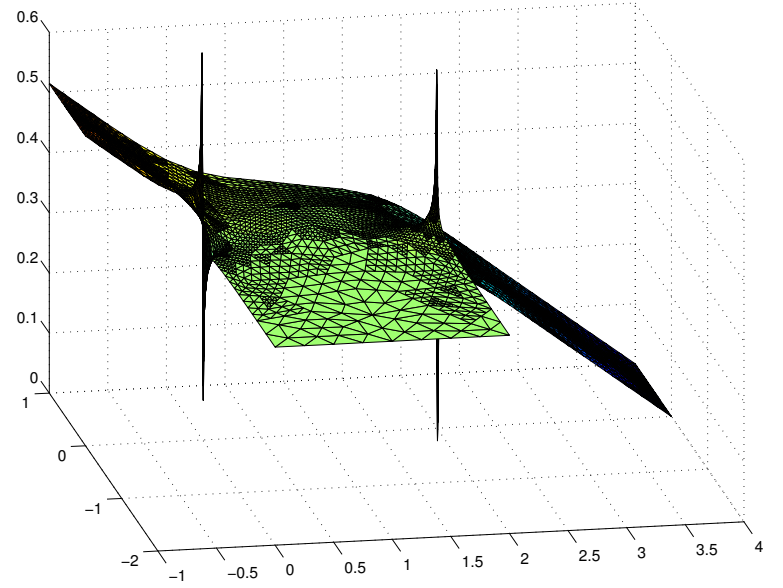

Figure 29: Adaptive problem 1, power-law, $r=3.3:$ pressure surface on the final mesh on the domain $\Omega_{2}$

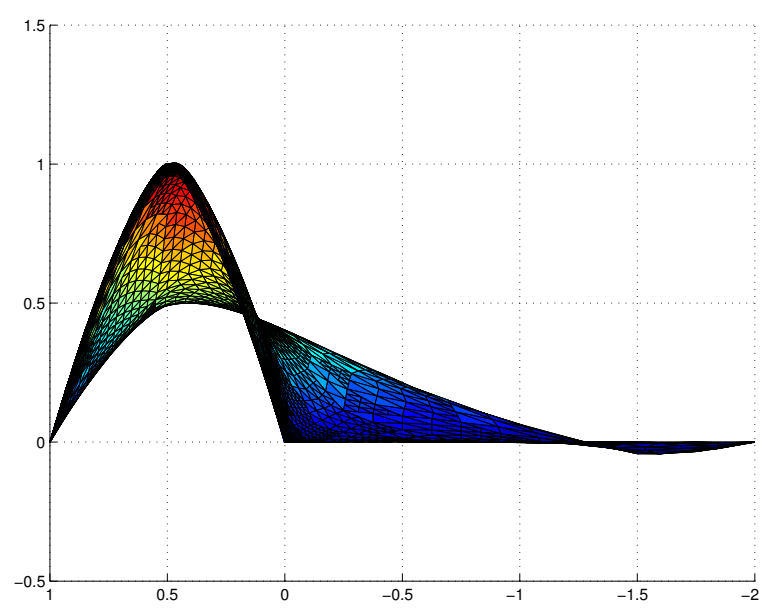

Figure 31: Adaptive problem 1, power-law, $r=3.3: u$ velocity component profile on the final mesh on the domain $\Omega_{2}$

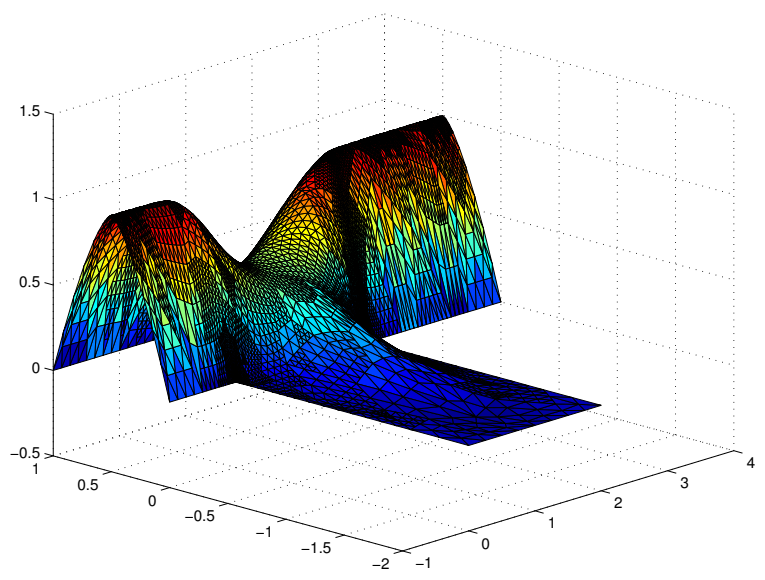

Figure 30: Adaptive problem 1, power-law, $r=3.3: u$ velocity component on the final mesh on the domain $\Omega_{2}$

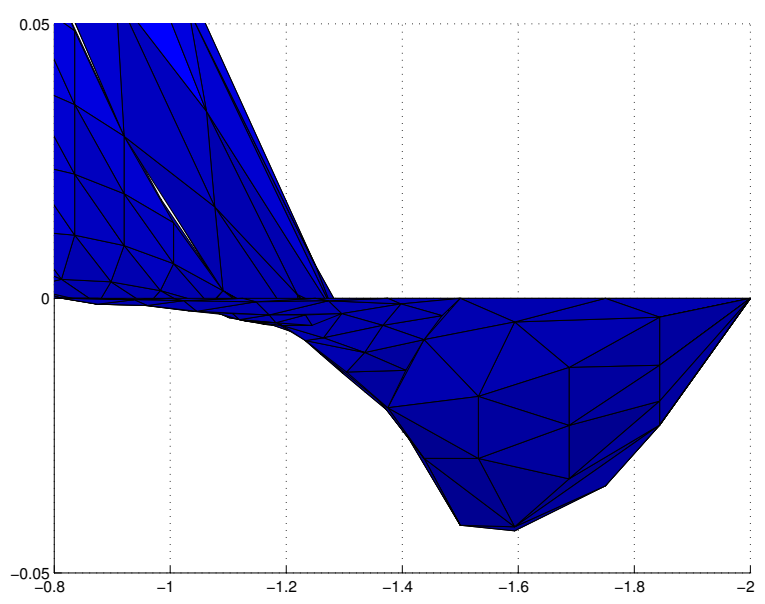

Figure 32: Adaptive problem 1, power-law, $r=3.3: u$ velocity component profile on the final mesh on the domain $\Omega_{2}$; detail in the lower part of the cavity 

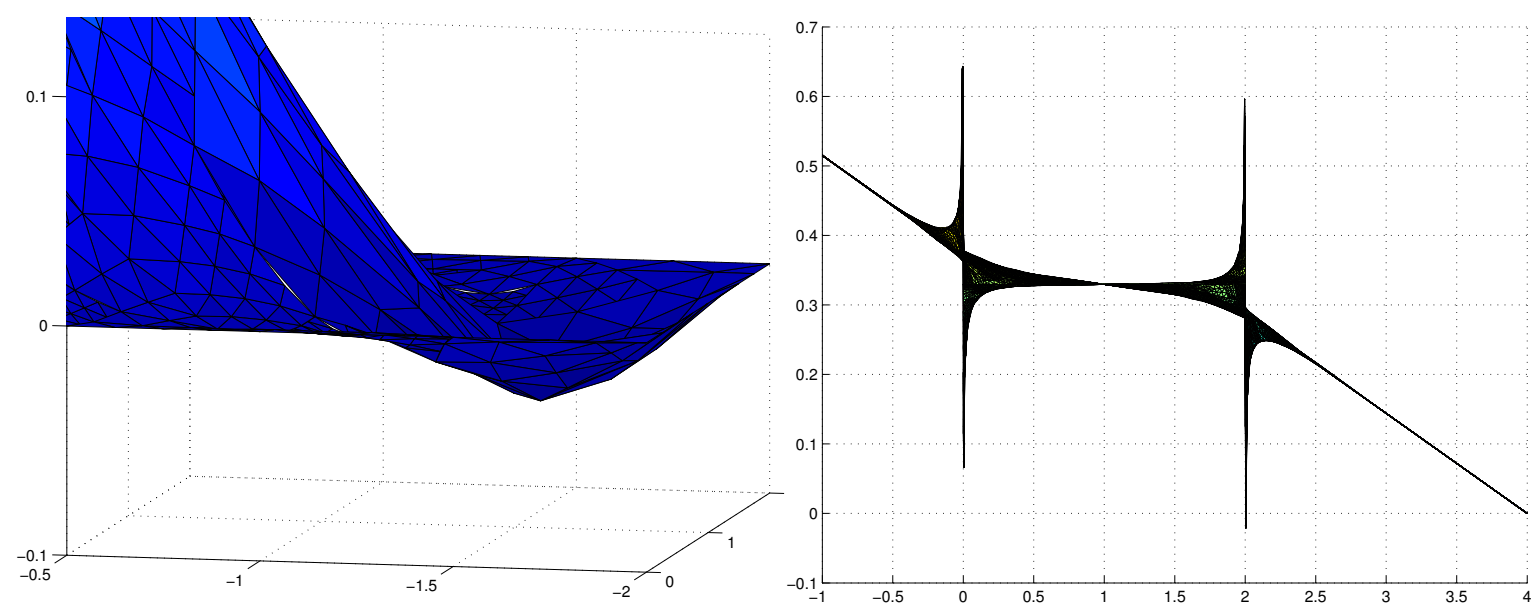

Figure 33: Adaptive problem 1, power-law, Figure 34: Adaptive problem 1, power-law, $r=3.3: u$ velocity component on the final $r=3.3$ : pressure profile on the final mesh mesh on the domain $\Omega_{2}$; detail in the lower on the domain $\Omega_{2}$ part of the cavity

can clearly see a strong refinement in the centre of the channel where the velocity profile of the fully developed flow displays large values of the higher derivatives (Figure 8) and a vanishing gradient giving a degenerate viscosity instead of a singularity of the viscosity coefficient as in the previous case of $r=1.3$.

In Figures 29 and 30 we report the pressure and the $u$ component of the velocity obtained on the final mesh on the domain $\Omega_{2}$.

In Figures 31-33 we show several details of the $u$ component of the velocity. The vortex in the lower part of the cavity is, in this case, much stronger than when $r=1.3$, but it does not display the same strong changes in curvature as for $r=1.3$. Moreover the slope of the $u$ component of the velocity is almost constant and nonzero in the transition region between positive values and negative ones. The velocity in this region seems to be easy to capture by our quadratic elements without requiring any special refinement.

In Figure 34 we report the pressure profile to show how the singularities in the upper corners of the cavity are well described on our final adapted mesh.

\subsection{Adaptive problem 2: Carreau law}

In this problem we consider the same geometries as in the previous problem, but we change the viscosity model to the Carreau law. The computations are performed with the following parameters: $\theta=1.3$ or $\theta=3.3, k_{\infty}=0, k_{0}=3.0 E-2, \lambda=1.0 E-4$. Instead of $k_{\infty}$ we consider the more interesting case with $k_{\infty}=0$ here, because we then have $r=\theta \neq 2$ and we can therefore observe a mismatch between the upper and the lower estimate. For the Carreau problem we cannot impose the exact solution for the fully developed flow at inflow and outflow, since it is unknown to us. Instead, we impose a parabolic velocity profile at the inflow and the associated homogeneous Neumann 
Table 11: Adaptive problem 2, Carreau law, $r=1.3, \Omega_{1}$ : error estimators during the adaptive process

\begin{tabular}{|c|c|c|c|c|c|}
\hline dofs & R.res & R.jump & R.cont & tot.R.U & tot.R.L \\
\hline 635 & $3.2467 \mathrm{E}-06$ & $4.9681 \mathrm{E}-07$ & $3.1933 \mathrm{E}-01$ & $3.2349 \mathrm{E}-01$ & $3.1933 \mathrm{E}-01$ \\
\hline 1517 & $2.5058 \mathrm{E}-06$ & $3.9348 \mathrm{E}-07$ & $1.2489 \mathrm{E}-01$ & $1.2859 \mathrm{E}-01$ & $1.2489 \mathrm{E}-01$ \\
\hline 2776 & $2.2925 \mathrm{E}-06$ & $3.5780 \mathrm{E}-07$ & $5.2025 \mathrm{E}-02$ & $5.5579 \mathrm{E}-02$ & $5.2028 \mathrm{E}-02$ \\
\hline 4177 & $2.2115 \mathrm{E}-06$ & $3.4362 \mathrm{E}-07$ & $2.4489 \mathrm{E}-02$ & $2.7982 \mathrm{E}-02$ & $2.4492 \mathrm{E}-02$ \\
\hline 5238 & $2.1263 \mathrm{E}-06$ & $3.3652 \mathrm{E}-07$ & $1.3776 \mathrm{E}-02$ & $1.7215 \mathrm{E}-02$ & $1.3779 \mathrm{E}-02$ \\
\hline 5983 & $1.9428 \mathrm{E}-06$ & $3.3118 \mathrm{E}-07$ & $9.7409 \mathrm{E}-03$ & $1.3074 \mathrm{E}-02$ & $9.7432 \mathrm{E}-03$ \\
\hline 6473 & $1.6579 \mathrm{E}-06$ & $3.2734 \mathrm{E}-07$ & $8.0746 \mathrm{E}-03$ & $1.1239 \mathrm{E}-02$ & $8.0766 \mathrm{E}-03$ \\
\hline
\end{tabular}

Table 12: Adaptive problem 2, Carreau law, $r=1.3, \Omega_{2}$ : error estimators during the adaptive process

\begin{tabular}{|c|c|c|c|c|c|}
\hline dofs & R.res & R.jump & R.cont & tot.R.U & tot.R.L \\
\hline 753 & $7.3488 \mathrm{E}-06$ & $6.2609 \mathrm{E}-07$ & $3.3244 \mathrm{E}-01$ & $3.3808 \mathrm{E}-01$ & $3.3245 \mathrm{E}-01$ \\
\hline 1780 & $3.9394 \mathrm{E}-06$ & $4.8279 \mathrm{E}-07$ & $1.2542 \mathrm{E}-01$ & $1.2984 \mathrm{E}-01$ & $1.2542 \mathrm{E}-01$ \\
\hline 3161 & $3.2687 \mathrm{E}-06$ & $4.3335 \mathrm{E}-07$ & $5.2211 \mathrm{E}-02$ & $5.6306 \mathrm{E}-02$ & $5.2215 \mathrm{E}-02$ \\
\hline 4740 & $2.9818 \mathrm{E}-06$ & $4.1090 \mathrm{E}-07$ & $2.3741 \mathrm{E}-02$ & $2.7686 \mathrm{E}-02$ & $2.3744 \mathrm{E}-02$ \\
\hline 6005 & $2.7517 \mathrm{E}-06$ & $3.9726 \mathrm{E}-07$ & $1.3171 \mathrm{E}-02$ & $1.6996 \mathrm{E}-02$ & $1.3174 \mathrm{E}-02$ \\
\hline 6841 & $2.4426 \mathrm{E}-06$ & $3.8740 \mathrm{E}-07$ & $9.1061 \mathrm{E}-03$ & $1.2773 \mathrm{E}-02$ & $9.1089 \mathrm{E}-03$ \\
\hline 7352 & $2.0055 \mathrm{E}-06$ & $3.7732 \mathrm{E}-07$ & $7.5109 \mathrm{E}-03$ & $1.0941 \mathrm{E}-02$ & $7.5133 \mathrm{E}-03$ \\
\hline
\end{tabular}

boundary condition at the outflow. The inconsistency between the flow that we have inside the outflow channel and the outflow boundary condition is responsible for the refinement at the outflow that we can observe in Figures 37, 38, 41, 42. We can clearly see that the region refined by the adaptive method around the cavity is detached from the refined region at the outflow.

\subsubsection{Case $\theta=r=1.3$}

First we take $\theta=r=1.3$. In Tables 11 and 12 we report the residuals and the error estimators, as in the previous cases. In Figures 35 and 36 we plot the last two columns of these tables and we can see that tot.R.U and tot.R.L tend to behave smoothly, though in a slightly different way, during the adaptive mesh-refinement process, due to the different powers of the residual and the jump-terms in the upper and lower bounds. 


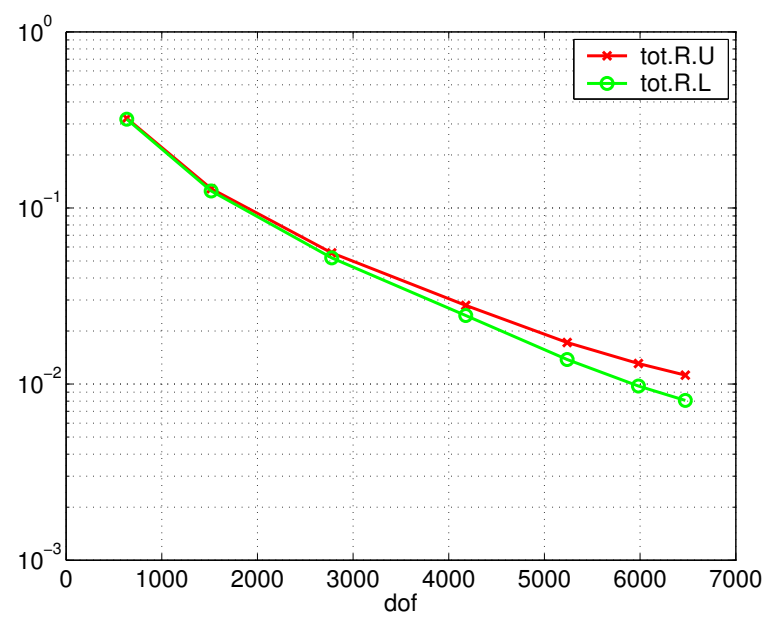

Figure 35: Adaptive problem 2, Carreau law, $r=1.3$ : total residuals during the adaptive process on $\Omega_{1}$

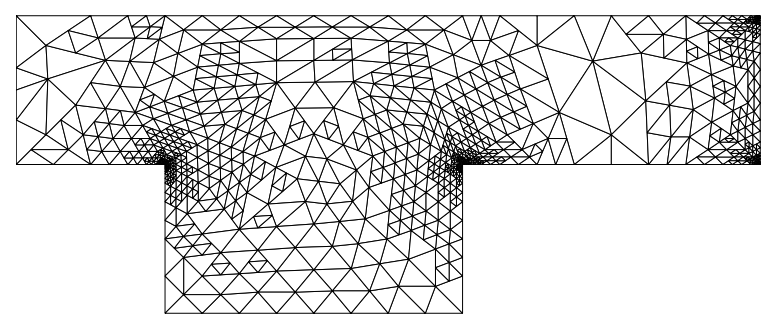

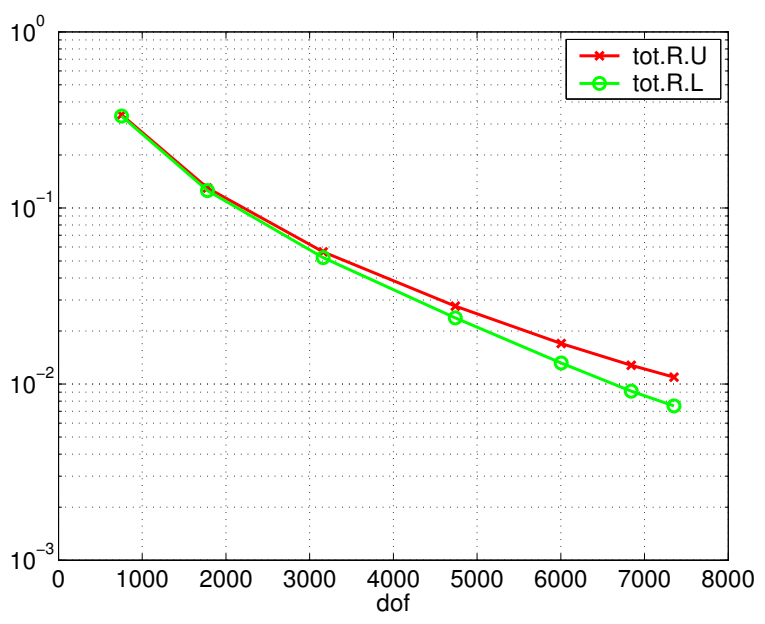

Figure 36: Adaptive problem 2, Carreau law, $r=1.3$ : total residuals during the adaptive process on $\Omega_{2}$

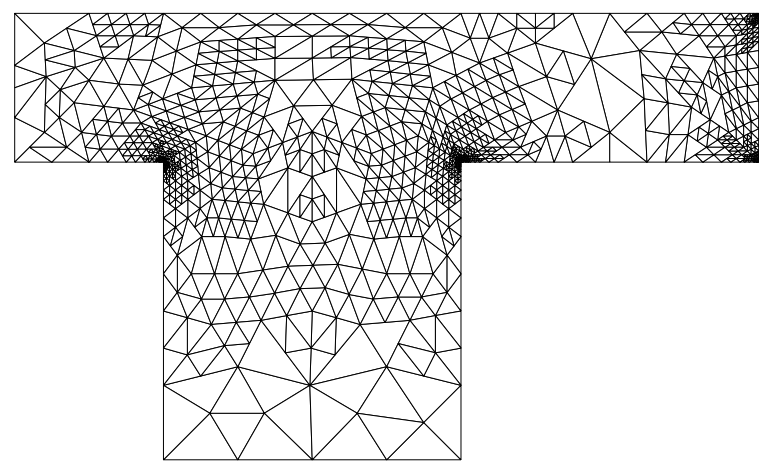

Figure 37: Adaptive problem 2, Carreau Figure 38: Adaptive problem 2, Carreau law, $r=1.3$ : final mesh on the domain $\Omega_{1}$ law, $r=1.3$ : final mesh on the domain $\Omega_{2}$ 
Table 13: Adaptive problem 2, Carreau law, $r=3.3, \Omega_{1}$ : error estimators during the adaptive process

\begin{tabular}{|c|c|c|c|c|c|}
\hline dofs & R.res & R.jump & R.cont & tot.R.U & tot.R.L \\
\hline 635 & $1.8150 \mathrm{E}-02$ & $1.3670 \mathrm{E}-02$ & $1.3975 \mathrm{E}-01$ & $3.3523 \mathrm{E}-01$ & $3.0967 \mathrm{E}-01$ \\
\hline 1378 & $8.5208 \mathrm{E}-03$ & $5.8908 \mathrm{E}-03$ & $9.5805 \mathrm{E}-02$ & $2.5577 \mathrm{E}-01$ & $2.4344 \mathrm{E}-01$ \\
\hline 2682 & $3.6463 \mathrm{E}-03$ & $2.5735 \mathrm{E}-03$ & $6.7845 \mathrm{E}-02$ & $2.0203 \mathrm{E}-01$ & $1.9645 \mathrm{E}-01$ \\
\hline 3997 & $1.8907 \mathrm{E}-03$ & $1.2514 \mathrm{E}-03$ & $4.8327 \mathrm{E}-02$ & $1.6256 \mathrm{E}-01$ & $1.5967 \mathrm{E}-01$ \\
\hline 5083 & $1.2081 \mathrm{E}-03$ & $7.5233 \mathrm{E}-04$ & $3.4401 \mathrm{E}-02$ & $1.3170 \mathrm{E}-01$ & $1.2987 \mathrm{E}-01$ \\
\hline 5825 & $9.5309 \mathrm{E}-04$ & $5.4282 \mathrm{E}-04$ & $2.4441 \mathrm{E}-02$ & $1.0696 \mathrm{E}-01$ & $1.0555 \mathrm{E}-01$ \\
\hline 6218 & $8.5824 \mathrm{E}-04$ & $4.6508 \mathrm{E}-04$ & $1.7275 \mathrm{E}-02$ & $8.6784 \mathrm{E}-02$ & $8.5537 \mathrm{E}-02$ \\
\hline
\end{tabular}

Table 14: Adaptive problem 2, Carreau law, $r=3.3, \Omega_{2}$ : error estimators during the adaptive process

\begin{tabular}{|c|c|c|c|c|c|}
\hline dofs & R.res & R.jump & R.cont & tot.R.U & tot.R.L \\
\hline 753 & $2.0934 \mathrm{E}-02$ & $1.5544 \mathrm{E}-02$ & $1.4227 \mathrm{E}-01$ & $3.4320 \mathrm{E}-01$ & $3.1430 \mathrm{E}-01$ \\
\hline 1701 & $8.6839 \mathrm{E}-03$ & $6.1584 \mathrm{E}-03$ & $9.7525 \mathrm{E}-02$ & $2.5882 \mathrm{E}-01$ & $2.4614 \mathrm{E}-01$ \\
\hline 3071 & $3.7983 \mathrm{E}-03$ & $2.6329 \mathrm{E}-03$ & $6.8755 \mathrm{E}-02$ & $2.0383 \mathrm{E}-01$ & $1.9807 \mathrm{E}-01$ \\
\hline 4582 & $1.9100 \mathrm{E}-03$ & $1.2637 \mathrm{E}-03$ & $4.8854 \mathrm{E}-02$ & $1.6364 \mathrm{E}-01$ & $1.6072 \mathrm{E}-01$ \\
\hline 5911 & $1.1582 \mathrm{E}-03$ & $7.0644 \mathrm{E}-04$ & $3.4759 \mathrm{E}-02$ & $1.3242 \mathrm{E}-01$ & $1.3068 \mathrm{E}-01$ \\
\hline 6732 & $8.8642 \mathrm{E}-04$ & $4.8792 \mathrm{E}-04$ & $2.4693 \mathrm{E}-02$ & $1.0750 \mathrm{E}-01$ & $1.0620 \mathrm{E}-01$ \\
\hline 7244 & $7.8052 \mathrm{E}-04$ & $4.0658 \mathrm{E}-04$ & $1.7386 \mathrm{E}-02$ & $8.6981 \mathrm{E}-02$ & $8.5859 \mathrm{E}-02$ \\
\hline
\end{tabular}

\subsubsection{Case $\theta=r=3.3$}

In this last case we only change $\theta$ and take $\theta=3.3$. Otherwise all parameters have the same values as in the previous experiment. In Tables 13 and 14 and in Figures 39 and 40 we report the residuals and the error estimators, as in the previous cases. Here the total residuals corresponding to the upper and the lower bounds do not display the mismatch seen for $r=1.3$. Figures 41 and 42 report the final meshes obtained by the adaptive process. We can see strong refinement around the corners of cavity and around the outflow edge due to the boundary condition.

\subsection{Adaptive problem 3}

Our final adaptive test-problem is Problem 1 considered in Section 6, again with the power-law model. The adaptive strategy we apply is the same as in the previous two problems of this section with the same adaptive parameters. In Tables 15 and 16 we compare the residuals with the exact errors and we report the effectivity indices as in 


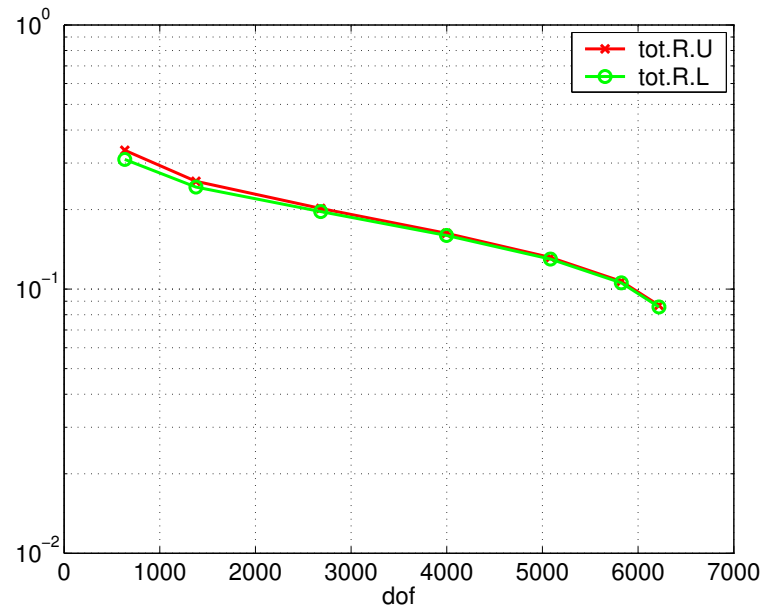

Figure 39: Adaptive problem 2, Carreau law, $r=3.3$ : total residuals during the adaptive process on $\Omega_{1}$

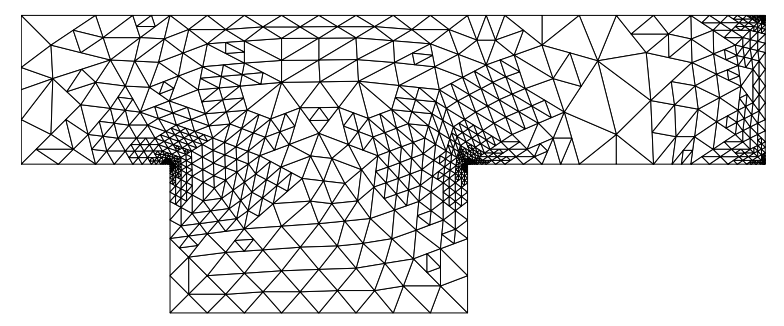

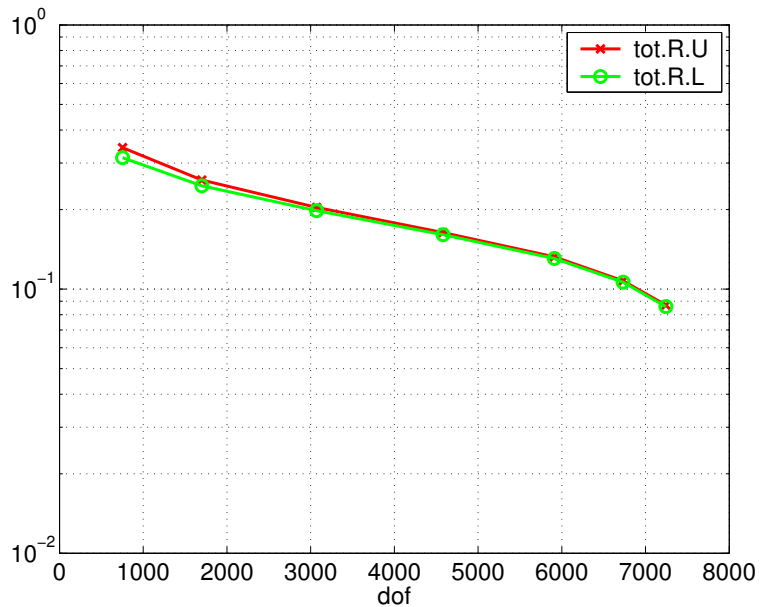

Figure 40: Adaptive problem 2, Carreau law, $r=3.3$ : total residuals during the adaptive process on $\Omega_{2}$

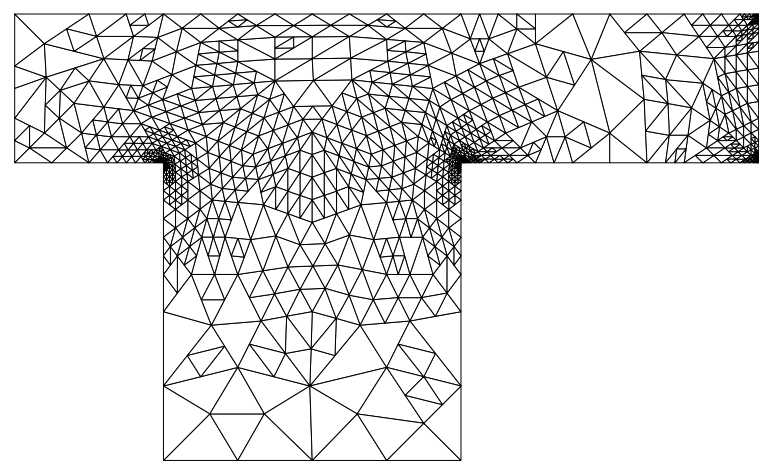

Figure 41: Adaptive problem 2, Carreau Figure 42: Adaptive problem 2, Carreau law, $r=3.3$ : final mesh on the domain $\Omega_{1}$ law, $r=3.3$ : final mesh on the domain $\Omega_{2}$

Table 15: Adaptive problem 3, $r=1.8$ : residuals, true errors and effectivity indices during the adaptive process

\begin{tabular}{|c|c|c|c|c|c|c|c|}
\hline dofs & R.res & R.jump & R.cont & E.Vh & E.ph & e.i.U & e.i.L \\
\hline 295 & 85.47 & 2.477 & $4.628 \mathrm{E}+5$ & $5.345 \mathrm{E}+5$ & 1.058 & 0.4473 & 0.9307 \\
\hline 700 & 5.665 & 0.09718 & $1.617 \mathrm{E}+4$ & $1.957 \mathrm{E}+4$ & 0.05453 & 0.525 & 0.909 \\
\hline 1523 & 0.9629 & 0.01355 & 2500 & 3059 & 0.007893 & 0.5789 & 0.9042 \\
\hline 2328 & 0.3489 & 0.003932 & 779 & 967.7 & 0.003183 & 0.6125 & 0.8974 \\
\hline 3265 & 0.1704 & 0.001871 & 350.2 & 441 & 0.001386 & 0.6356 & 0.8914 \\
\hline 3908 & 0.1183 & $9.977 \mathrm{E}-4$ & 207.8 & 264.8 & $8.91 \mathrm{E}-4$ & 0.65 & 0.8861 \\
\hline 4270 & 0.09899 & $7.506 \mathrm{E}-4$ & 143.9 & 184.9 & $6.873 \mathrm{E}-4$ & 0.6605 & 0.8826 \\
\hline 4511 & 0.08815 & $7.031 \mathrm{E}-4$ & 133.9 & 171.9 & $6.23 \mathrm{E}-4$ & 0.6634 & 0.8829 \\
\hline
\end{tabular}


Table 16: Adaptive problem 3, $r=3.0$ : residuals, true errors and effectivity indices during the adaptive process

\begin{tabular}{|c|c|c|c|c|c|c|c|}
\hline dofs & R.res & R.jump & R.cont & E.Vh & E.ph & e.i.U & e.i.L \\
\hline 295 & 38.67 & 6.793 & $1.045 \mathrm{E}+4$ & 7471 & 1.524 & 0.2647 & 1.273 \\
\hline 716 & 5.533 & 0.8902 & 801.6 & 632.9 & 0.241 & 0.3827 & 1.146 \\
\hline 1471 & 1.283 & 0.1366 & 99.41 & 67.78 & 0.04777 & 0.5809 & 1.174 \\
\hline 2527 & 0.6185 & 0.05649 & 30 & 20.81 & 0.02137 & 0.7045 & 1.161 \\
\hline 3625 & 0.3794 & 0.03065 & 9.625 & 6.879 & 0.01231 & 0.8468 & 1.153 \\
\hline 4422 & 0.286 & 0.02421 & 7.138 & 5.026 & 0.01007 & 0.8939 & 1.153 \\
\hline 5106 & 0.2339 & 0.01914 & 4.797 & 3.345 & 0.008414 & 0.9621 & 1.157 \\
\hline 5487 & 0.2097 & 0.01566 & 4.046 & 2.747 & 0.007243 & 1.003 & 1.166 \\
\hline
\end{tabular}

Section 6 , for the two values $r=1.8$ and $r=3.0$, with $\mu=1.0 E-3$. We can see in Table 16 that the lower effectivity index e.i.L and the upper effectivity index e.i.U are both quite close to 1 .

\section{Approximate computation of $\left\|h_{T} \prod_{T} \mathbf{R}_{T}\left(\left[\mathbf{u}_{h}, p_{h}\right]\right)\right\|_{L^{r^{\prime}}(T)}$}

In this section we wish to propose an heuristic approximation of the norm of the residual in each triangle that avoids the computation of second derivatives of the approximate solution and of first derivatives of the viscosity function $k($.$) required in the computation$ of $\nabla \cdot \sigma$ by the product rule for differentiation. This approximation can be useful when the viscosity model is the power-law and the computational domain contains large regions where the gradient of the solution is very small (which is usually the case when $1<r<$ 2 ). The approximation is based on the inequalities (5.7) that imply

$$
\left\|h_{T} \Pi_{T} \mathbf{R}_{T}\left(\left[\mathbf{u}_{h}, p_{h}\right]\right)\right\|_{L^{r^{\prime}(T)}} \asymp \sup _{v \in \mathrm{V}_{T}} \frac{\left|\int_{T} h_{T} \Pi_{T} \mathbf{R}_{T}\left(\left[\mathbf{u}_{h}, p_{h}\right]\right) b_{T} v \mathrm{~d} \Omega\right|}{\left\|b_{T} v\right\|_{L^{r}(T)}} .
$$

The previous relation holds for every finite-dimensional function space $V_{T}$ such that $\Pi_{T} \mathbf{R}_{T}\left(\left[\mathbf{u}_{h}, p_{h}\right]\right) \in \mathrm{V}_{T}$. We can avoid the computation of the first and second derivatives referred to above by observing that

$$
\begin{aligned}
\int_{T} \Pi_{T} h_{T} \mathbf{R}_{T}\left(\left[\mathbf{u}_{h}, p_{h}\right]\right) b_{T} v \mathrm{~d} \Omega & =\int_{T} h_{T} \mathbf{R}_{T}\left(\left[\mathbf{u}_{h}, p_{h}\right]\right) b_{T} v \mathrm{~d} \Omega \\
+h_{T} & \left(\int_{T} \Pi_{T} \mathbf{R}_{T}\left(\left[\mathbf{u}_{h}, p_{h}\right]\right) b_{T} v \mathrm{~d} \Omega-\int_{T} \mathbf{R}_{T}\left(\left[\mathbf{u}_{h}, p_{h}\right]\right) b_{T} v \mathrm{~d} \Omega\right) \\
& \sim \int_{T} h_{T}\left(\mathbf{f}+\nabla \cdot \sigma_{h}\right) b_{T} v \mathrm{~d} \Omega \\
& =\int_{T} h_{T}\left(\mathbf{f} b_{T} v-\sigma_{h}: \nabla\left(b_{T} v\right)\right) \mathrm{d} \Omega
\end{aligned}
$$


obtained by integration by parts and exploiting the fact that $b_{T}$ vanishes on $\partial T$; here $\sigma_{h}=k\left(\left|e\left(\mathbf{u}_{h}\right)\right|\right) e\left(\mathbf{u}_{h}\right)-p_{h} I$. Noting that

$$
\begin{aligned}
& \int_{T} h_{T}\left(\mathbf{f} b_{T} v-\sigma_{h}: \nabla\left(b_{T} v\right)\right) \mathrm{d} \Omega=\int_{T} h_{T} \Pi_{T}\left(\mathbf{f} b_{T} v-\sigma_{h}: \nabla\left(b_{T} v\right)\right) \mathrm{d} \Omega \\
&+h_{T}\left(\int_{T}\left(\mathbf{f} b_{T} v-\sigma_{h}: \nabla\left(b_{T} v\right)\right) \mathrm{d} \Omega-\int_{T} \Pi_{T}\left(\mathbf{f} b_{T} v-\sigma_{h}: \nabla\left(b_{T} v\right)\right)\right) \\
& \sim \int_{T} h_{T} \Pi_{T}\left(\mathbf{f} b_{T} v-\sigma_{h}: \nabla\left(b_{T} v\right)\right)
\end{aligned}
$$

then motivates us to use the following approximation:

$$
\left\|h_{T} \Pi_{T} \mathbf{R}_{T}\left(\left[\mathbf{u}_{h}, p_{h}\right]\right)\right\|_{L^{r^{\prime}}(T)} \sim \frac{\left|\int_{T} h_{T} \Pi_{T}\left(\mathbf{f} b_{T}-\sigma_{h} \nabla b_{T}\right) \mathrm{d} \Omega\right|}{\left\|b_{T}\right\|_{L^{r}(T)}},
$$

which can be viewed as an approximation of the norm of the residual if the residual is constant on each element.

In what follows, we shall compare the residuals obtained by the exact and this approximate computation of $\left\|h_{T} \Pi_{T} \mathbf{R}_{T}\left(\left[\mathbf{u}_{h}, p_{h}\right]\right)\right\|_{L^{r^{\prime}}(T)}$ for power-law flows with $r=1.3$ and $r=3.3$. When $1<r<2$ the viscosity coefficient $k($.$) is singular and sometimes the$ internal residual can display strong oscillations in parts of the computational domain where the velocity gradient vanishes; this is, most probably, due to the fact that in the computation of the residual we need to evaluate the quantity $k\left(\left|\nabla \mathbf{u}_{h}\right|\right)=2 \mu\left|\nabla \mathbf{u}_{h}\right|^{r-2}$ and its partial derivatives in these regions. We present some computations performed with the following parameters: $\Omega_{1}, \mu=5.0 E-2, r=1.3$, with the initial refinement percentage set to $40 \%$, the initial coarsening percentage set to $4 \%$ and the reduction-factor in these percentages equal to 3 in each refinement step.

As before, In Tables 17 and 18 we compare the residuals during the adaptive steps obtained by exact computation of the norm of the residual on the one hand and with approximate computation of the norm of the residual as proposed above on the other.

In Figures 43 and 44 we compare the final meshes obtained with the two methods. The meshes are quite similar, but the approximate computation of the residuals gives cleaner meshes in the outflow channel where, despite the singularity of $k($.$) , the solution$ is quite easy to capture on a grid that is coarse around the centre-line of the channel.

In Figures 45 and 46 we compare the total residuals during the adaptive process and in Figures 47, 48 and 49 we plot the maximum over the triangles of all the residuals, their mean-values and their root-mean-square (r.m.s.). We can notice the markedly oscillatory behaviour of the exactly computed residuals.

In the following we report the corresponding tables and figures obtained with the same parameters but on the domain $\Omega_{2}$. In Tables 19 and 20 we compare the residuals during the adaptive steps. In Figures 50 and 51 we compare the final meshes obtained with the two methods.

In Figures 52 and 53 we compare the total residuals during the adaptive process. Clearly, a more regular behaviour of the total residuals is observed during the adaptive process when we use the approximation proposed above. In Figures 54, 55 and 56 we 
Table 17: Adaptive problem 1, power-law, $r=1.3, \Omega_{1}$ : error estimators during the adaptive process, exact computation of the norm of residuals

\begin{tabular}{|c|c|c|c|c|c|}
\hline dofs & R.res & R.jump & R.cont & tot.R.U & tot.R.L \\
\hline 635 & $2.9191 \mathrm{E}+00$ & $7.1759 \mathrm{E}-05$ & $4.5333 \mathrm{E}-01$ & $2.1051 \mathrm{E}+00$ & $3.3725 \mathrm{E}+00$ \\
\hline 1479 & $4.8889 \mathrm{E}-02$ & $2.5823 \mathrm{E}-05$ & $2.3615 \mathrm{E}-01$ & $4.9211 \mathrm{E}-01$ & $2.8507 \mathrm{E}-01$ \\
\hline 2792 & $5.5945 \mathrm{E}-02$ & $9.1856 \mathrm{E}-06$ & $1.2777 \mathrm{E}-01$ & $3.9677 \mathrm{E}-01$ & $1.8372 \mathrm{E}-01$ \\
\hline 3854 & $6.3930 \mathrm{E}-03$ & $3.5964 \mathrm{E}-06$ & $7.6642 \mathrm{E}-02$ & $1.7682 \mathrm{E}-01$ & $8.3039 \mathrm{E}-02$ \\
\hline 4249 & $1.2689 \mathrm{E}-03$ & $2.2270 \mathrm{E}-06$ & $5.7644 \mathrm{E}-02$ & $1.0614 \mathrm{E}-01$ & $5.8915 \mathrm{E}-02$ \\
\hline 4370 & $8.6905 \mathrm{E}-04$ & $1.7808 \mathrm{E}-06$ & $5.1195 \mathrm{E}-02$ & $9.2074 \mathrm{E}-02$ & $5.2066 \mathrm{E}-02$ \\
\hline 4452 & $2.4884 \mathrm{E}-04$ & $1.1530 \mathrm{E}-06$ & $4.8946 \mathrm{E}-02$ & $7.2469 \mathrm{E}-02$ & $4.9196 \mathrm{E}-02$ \\
\hline
\end{tabular}

Table 18: Adaptive problem 1, power-law, $r=1.3, \Omega_{1}$ : error estimators during the adaptive process, approximate computation of the norm of residuals

\begin{tabular}{|c|c|c|c|c|c|}
\hline dofs & R.res & R.jump & R.cont & tot.R.U & tot.R.L \\
\hline 635 & $5.6523 \mathrm{E}-04$ & $7.1759 \mathrm{E}-05$ & $4.5333 \mathrm{E}-01$ & $4.9726 \mathrm{E}-01$ & $4.5397 \mathrm{E}-01$ \\
\hline 1525 & $1.1391 \mathrm{E}-04$ & $2.5889 \mathrm{E}-05$ & $2.3435 \mathrm{E}-01$ & $2.5712 \mathrm{E}-01$ & $2.3449 \mathrm{E}-01$ \\
\hline 2733 & $2.0579 \mathrm{E}-05$ & $9.0263 \mathrm{E}-06$ & $1.2840 \mathrm{E}-01$ & $1.3996 \mathrm{E}-01$ & $1.2843 \mathrm{E}-01$ \\
\hline 3579 & $9.7671 \mathrm{E}-06$ & $3.8928 \mathrm{E}-06$ & $7.7996 \mathrm{E}-02$ & $8.6052 \mathrm{E}-02$ & $7.8009 \mathrm{E}-02$ \\
\hline 3906 & $6.4137 \mathrm{E}-06$ & $2.5198 \mathrm{E}-06$ & $6.0753 \mathrm{E}-02$ & $6.7371 \mathrm{E}-02$ & $6.0762 \mathrm{E}-02$ \\
\hline 4032 & $4.1799 \mathrm{E}-06$ & $1.8161 \mathrm{E}-06$ & $5.3974 \mathrm{E}-02$ & $5.9506 \mathrm{E}-02$ & $5.3980 \mathrm{E}-02$ \\
\hline 4098 & $3.0807 \mathrm{E}-06$ & $1.3769 \mathrm{E}-06$ & $5.1659 \mathrm{E}-02$ & $5.6491 \mathrm{E}-02$ & $5.1664 \mathrm{E}-02$ \\
\hline
\end{tabular}

plot the maximum of all the residuals, their mean-values and their root-mean-square; again we can see that the approximate computation of the norm of the internal residual in each element leads to a more regular decrease of all quantities considered. Moreover, in these cases, using this approximation, the values of the residuals in each element are of the same order of magnitude as the jumps, and in this way an adaptive method based on the values of $R_{m o m, T}$ takes into account in roughly equal measure the two contributions from the momentum equation to the total residual.

We conclude this section by reporting some results obtained on the domain $\Omega_{2}$ with $r=3.3$, all other parameters being kept fixed.

In Tables 21 and 22 we compare the residuals during the adaptive steps. In Figures 57 and 58 we compare the total residuals during the adaptive process. We clearly observe that in this case there isn't a noteworthy difference between the approximate computation of the norm of the residual inside each element and its exact computation. In Figures 59, 60 and 61 we plot the maximum, the mean-values and the rootmean-square of all the residuals; again the approximation and the exact computation of $\left\|h_{T} \Pi_{T} \mathbf{R}_{T}\left(\left[\mathbf{u}_{h}, p_{h}\right]\right)\right\|_{L^{r^{\prime}}(T)}$ lead to very similar behaviour. 


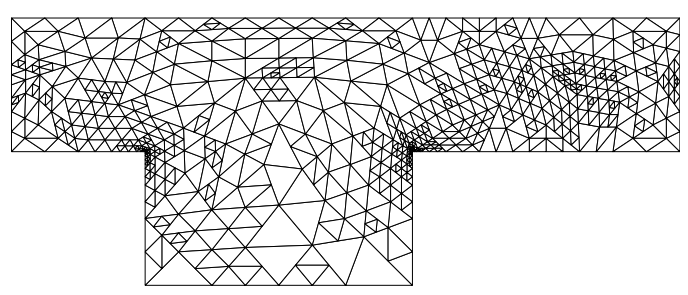

Figure 43: Adaptive problem 1, power-law $r=1.3, \Omega_{1}$ : final mesh, exact computation of the norm of the residual

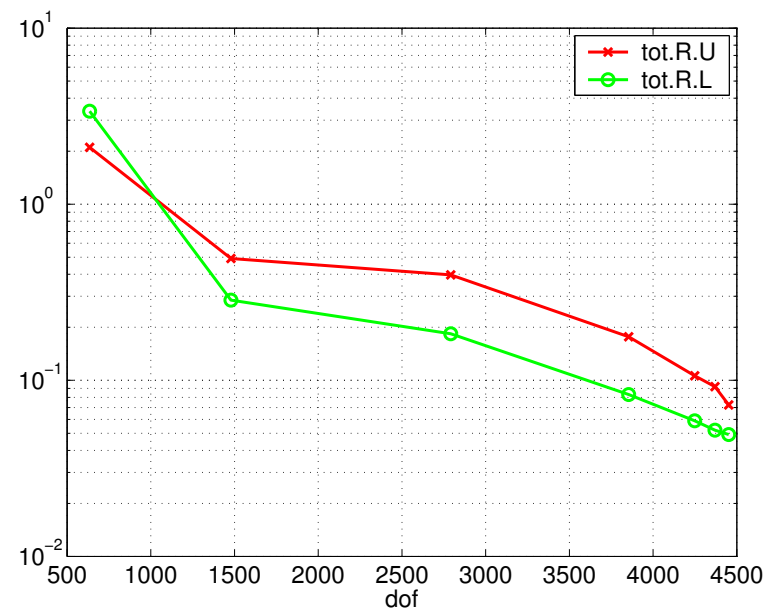

Figure 45: Adaptive problem 1, power-law, Figure 46: Adaptive problem 1, power-law, $r=1.3, \Omega_{1}$ : total residuals during the $r=1.3, \Omega_{1}$ : total residuals during the adaptive process with exact computation of the norm of the residual

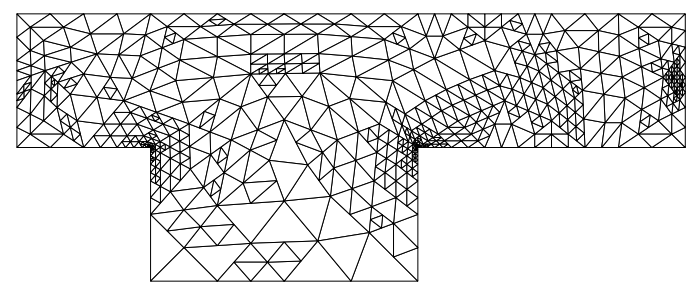

Figure 44: Adaptive problem 1, power-law, $r=1.3, \Omega_{1}$ : final mesh, approximate computation of the norm of the residual

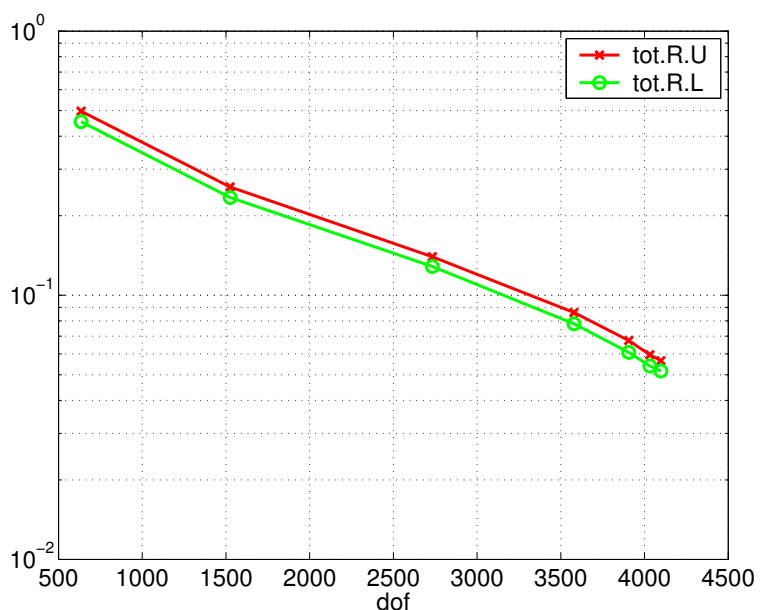

adaptive process with approximate computation of the norm of the residual

Table 19: Adaptive problem 1, power-law, $r=1.3, \Omega_{2}$ : error estimators during the adaptive process, exact computation of the norm of residuals

\begin{tabular}{|c|c|c|c|c|c|}
\hline dofs & R.res & R.jump & R.cont & tot.R.U & tot.R.L \\
\hline 753 & $2.3691 \mathrm{E}+00$ & $7.6790 \mathrm{E}-05$ & $4.5948 \mathrm{E}-01$ & $1.9611 \mathrm{E}+00$ & $2.8287 \mathrm{E}+00$ \\
\hline 1876 & $5.8274 \mathrm{E}+02$ & $2.7811 \mathrm{E}-05$ & $2.2828 \mathrm{E}-01$ & $1.9132 \mathrm{E}+01$ & $5.8297 \mathrm{E}+02$ \\
\hline 3526 & $7.3828 \mathrm{E}-03$ & $8.6622 \mathrm{E}-06$ & $1.1770 \mathrm{E}-01$ & $2.2609 \mathrm{E}-01$ & $1.2510 \mathrm{E}-01$ \\
\hline 4657 & $4.2256 \mathrm{E}-03$ & $3.3978 \mathrm{E}-06$ & $6.6718 \mathrm{E}-02$ & $1.4993 \mathrm{E}-01$ & $7.0947 \mathrm{E}-02$ \\
\hline 5139 & $3.6371 \mathrm{E}-03$ & $1.8743 \mathrm{E}-06$ & $4.8855 \mathrm{E}-02$ & $1.2598 \mathrm{E}-01$ & $5.2493 \mathrm{E}-02$ \\
\hline 5330 & $6.2730 \mathrm{E}-04$ & $1.4039 \mathrm{E}-06$ & $4.1957 \mathrm{E}-02$ & $7.7206 \mathrm{E}-02$ & $4.2586 \mathrm{E}-02$ \\
\hline 5444 & $5.2900 \mathrm{E}-02$ & $7.4092 \mathrm{E}-07$ & $3.9338 \mathrm{E}-02$ & $2.9835 \mathrm{E}-01$ & $9.2239 \mathrm{E}-02$ \\
\hline
\end{tabular}



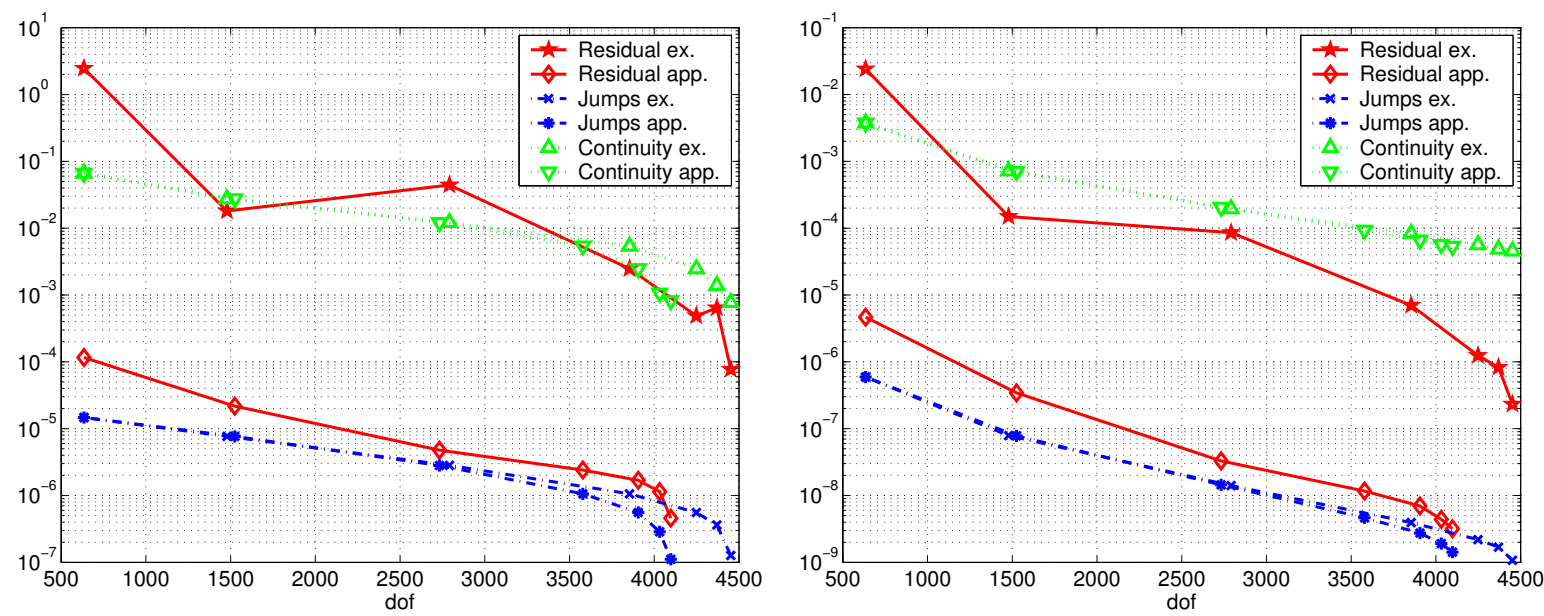

Figure 47: Adaptive problem 1, power-law, Figure 48: Adaptive problem 1, power-law, $r=1.3, \Omega_{1}$, comparison of the maximal $r=1.3, \Omega_{1}$, comparison of the mean-values values of the residuals on the elements of the residuals on the elements

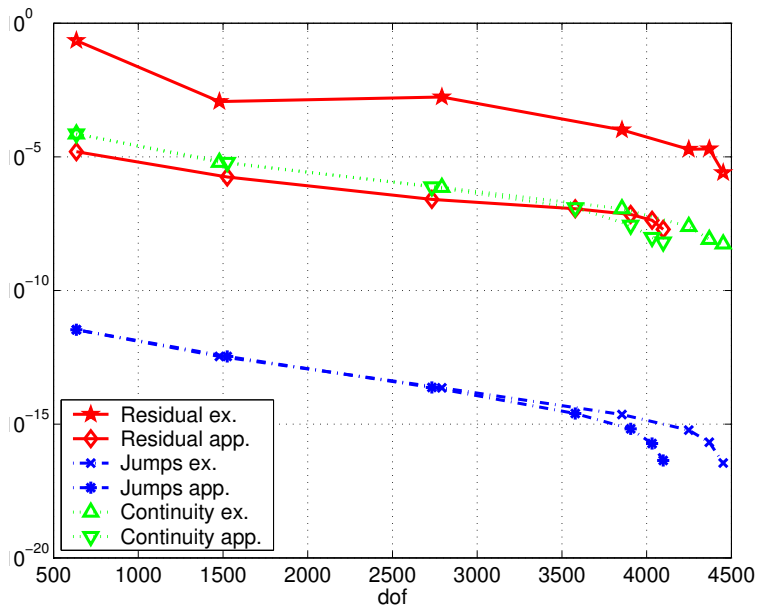

Figure 49: Adaptive problem 1, power-law, $r=1.3, \Omega_{1}$, comparison of the r.m.s. values of the residuals on the elements 
Table 20: Adaptive problem 1, power-law, $r=1.3, \Omega_{2}$ : error estimators during the adaptive process, approximate computation of the norm of residuals

\begin{tabular}{|c|c|c|c|c|c|}
\hline dofs & R.res & R.jump & R.cont & tot.R.U & tot.R.L \\
\hline 753 & $5.3628 \mathrm{E}-04$ & $7.6790 \mathrm{E}-05$ & $4.5948 \mathrm{E}-01$ & $5.0303 \mathrm{E}-01$ & $4.6009 \mathrm{E}-01$ \\
\hline 1836 & $9.0085 \mathrm{E}-05$ & $2.7704 \mathrm{E}-05$ & $2.3103 \mathrm{E}-01$ & $2.5249 \mathrm{E}-01$ & $2.3114 \mathrm{E}-01$ \\
\hline 3205 & $2.4234 \mathrm{E}-05$ & $9.0347 \mathrm{E}-06$ & $1.1825 \mathrm{E}-01$ & $1.3036 \mathrm{E}-01$ & $1.1828 \mathrm{E}-01$ \\
\hline 4209 & $1.1778 \mathrm{E}-05$ & $3.6115 \mathrm{E}-06$ & $6.7637 \mathrm{E}-02$ & $7.6024 \mathrm{E}-02$ & $6.7652 \mathrm{E}-02$ \\
\hline 4590 & $6.0203 \mathrm{E}-06$ & $2.1222 \mathrm{E}-06$ & $5.0265 \mathrm{E}-02$ & $5.6569 \mathrm{E}-02$ & $5.0273 \mathrm{E}-02$ \\
\hline 4700 & $3.9801 \mathrm{E}-06$ & $1.4403 \mathrm{E}-06$ & $4.2932 \mathrm{E}-02$ & $4.8163 \mathrm{E}-02$ & $4.2937 \mathrm{E}-02$ \\
\hline 4766 & $2.4446 \mathrm{E}-06$ & $1.2373 \mathrm{E}-06$ & $4.0850 \mathrm{E}-02$ & $4.5297 \mathrm{E}-02$ & $4.0854 \mathrm{E}-02$ \\
\hline
\end{tabular}
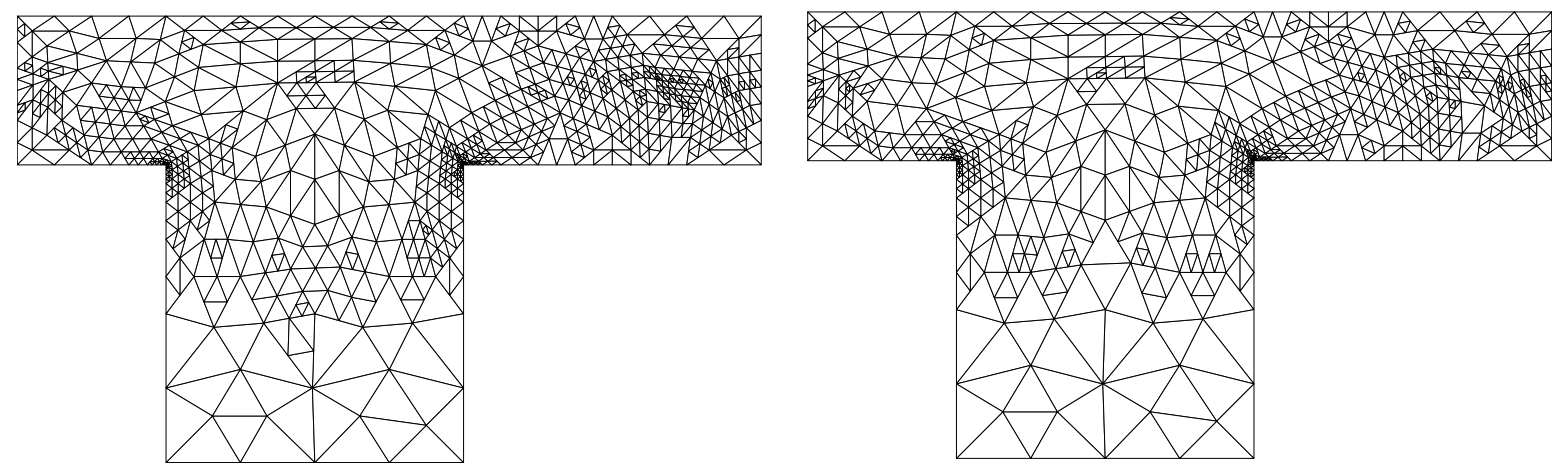

Figure 50: Adaptive problem 1, power-law, Figure 51: Adaptive problem 1, power-law, $r=1.3, \Omega_{2}$ : final mesh, exact computation of the norm of the residual putation of the norm of the residual
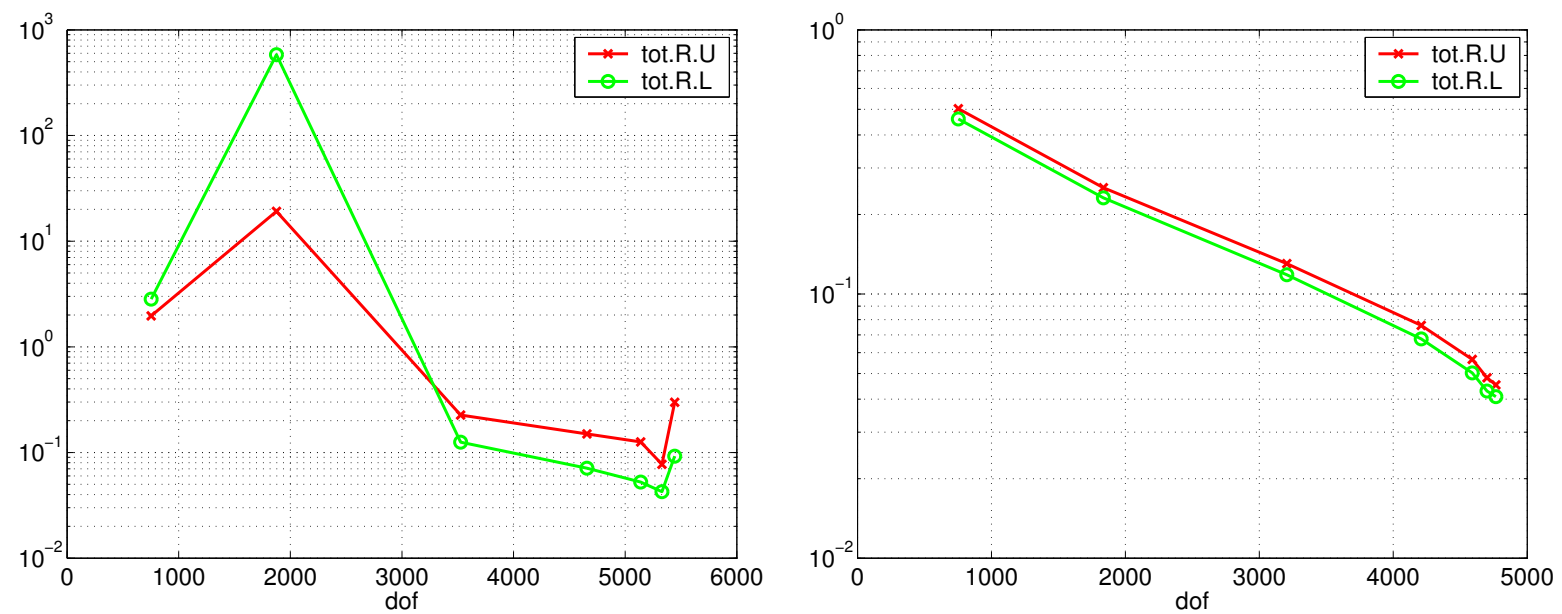

Figure 52: Adaptive problem 1, power-law, Figure 53: Adaptive problem 1, power-law, $r=1.3:$ total residuals during the adaptive process on $\Omega_{2}$ with exact computation of the norm of the residual $r=1.3:$ total residuals during the adaptive process on $\Omega_{2}$ with approximate computation of the norm of the residual 


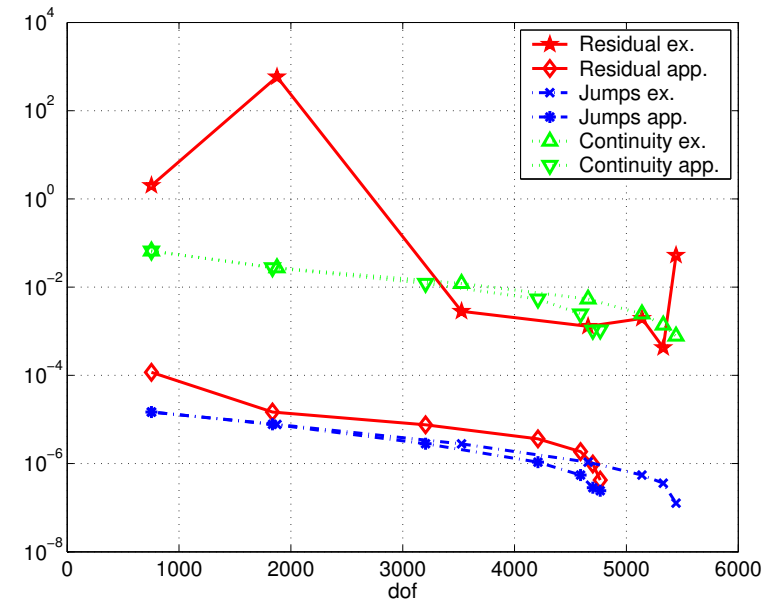

Figure 54: Adaptive problem 1, power-law, Figure 55: Adaptive problem 1, power-law, $r=1.3, \Omega_{2}$, comparison of the maximal val- $r=1.3, \Omega_{2}$, comparison of the mean-values ues of the residuals on the elements

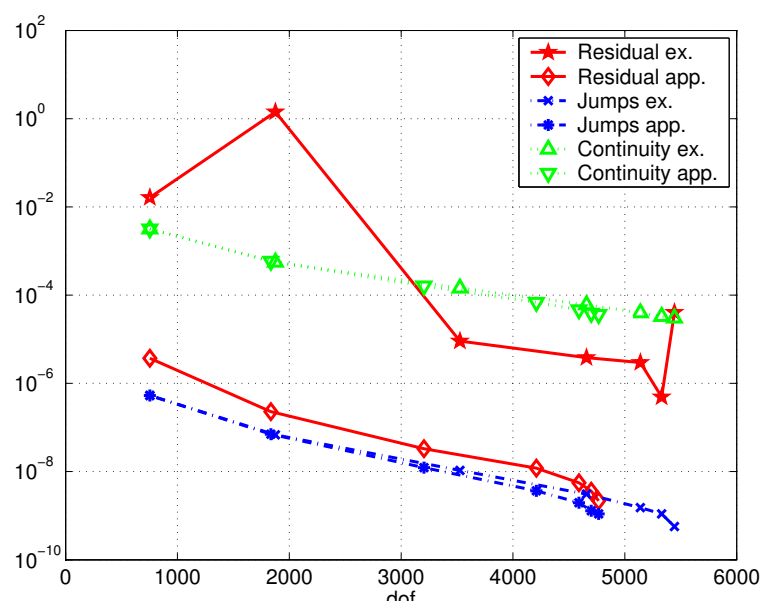

of the residuals on the elements

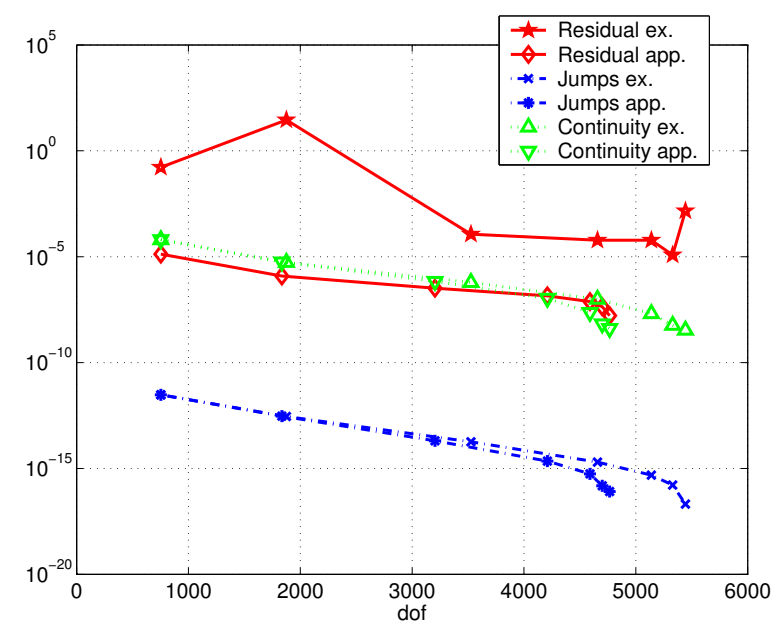

Figure 56: Adaptive problem 1, power-law, $r=1.3, \Omega_{2}$, comparison of the r.m.s. values of the residuals on the elements 
Table 21: Adaptive problem 1, power-law, $r=3.3, \Omega_{2}$ : error estimators during the adaptive process, exact computation of the norm of residuals

\begin{tabular}{|c|c|c|c|c|c|}
\hline dofs & R.res & R.jump & R.cont & tot.R.U & tot.R.L \\
\hline 753 & $4.4480 \mathrm{E}-01$ & $1.8540 \mathrm{E}-01$ & $5.1408 \mathrm{E}-02$ & $7.9570 \mathrm{E}-01$ & $5.8422 \mathrm{E}-01$ \\
\hline 1735 & $1.3521 \mathrm{E}-01$ & $7.1885 \mathrm{E}-02$ & $2.3395 \mathrm{E}-02$ & $3.0980 \mathrm{E}-01$ & $1.8966 \mathrm{E}-01$ \\
\hline 2765 & $6.1687 \mathrm{E}-02$ & $3.2629 \mathrm{E}-02$ & $1.0412 \mathrm{E}-02$ & $1.5719 \mathrm{E}-01$ & $9.1938 \mathrm{E}-02$ \\
\hline 3597 & $3.9233 \mathrm{E}-02$ & $1.9285 \mathrm{E}-02$ & $4.8769 \mathrm{E}-03$ & $9.8226 \mathrm{E}-02$ & $5.4734 \mathrm{E}-02$ \\
\hline 3915 & $3.2138 \mathrm{E}-02$ & $1.4516 \mathrm{E}-02$ & $2.3732 \mathrm{E}-03$ & $7.2317 \mathrm{E}-02$ & $3.6698 \mathrm{E}-02$ \\
\hline 3992 & $3.0427 \mathrm{E}-02$ & $1.2860 \mathrm{E}-02$ & $1.4677 \mathrm{E}-03$ & $6.2465 \mathrm{E}-02$ & $2.9179 \mathrm{E}-02$ \\
\hline 4026 & $2.9346 \mathrm{E}-02$ & $1.2016 \mathrm{E}-02$ & $1.1904 \mathrm{E}-03$ & $5.8254 \mathrm{E}-02$ & $2.6307 \mathrm{E}-02$ \\
\hline
\end{tabular}

Table 22: Adaptive problem 1, power-law, $r=3.3, \Omega_{2}$ : error estimators during the adaptive process, approximate computation of the norm of residuals

\begin{tabular}{|c|c|c|c|c|c|}
\hline dofs & R.res & R.jump & R.cont & tot.R.U & tot.R.L \\
\hline 753 & $9.9332 \mathrm{E}-02$ & $1.8540 \mathrm{E}-01$ & $5.1408 \mathrm{E}-02$ & $4.5024 \mathrm{E}-01$ & $3.0095 \mathrm{E}-01$ \\
\hline 1767 & $4.8424 \mathrm{E}-02$ & $7.1868 \mathrm{E}-02$ & $2.3395 \mathrm{E}-02$ & $2.2299 \mathrm{E}-01$ & $1.4287 \mathrm{E}-01$ \\
\hline 2729 & $2.4523 \mathrm{E}-02$ & $3.3637 \mathrm{E}-02$ & $1.0424 \mathrm{E}-02$ & $1.2108 \mathrm{E}-01$ & $7.7455 \mathrm{E}-02$ \\
\hline 3473 & $1.5932 \mathrm{E}-02$ & $1.9903 \mathrm{E}-02$ & $4.9006 \mathrm{E}-03$ & $7.5660 \mathrm{E}-02$ & $4.7198 \mathrm{E}-02$ \\
\hline 3835 & $1.2693 \mathrm{E}-02$ & $1.4986 \mathrm{E}-02$ & $2.3889 \mathrm{E}-03$ & $5.3444 \mathrm{E}-02$ & $3.0901 \mathrm{E}-02$ \\
\hline 3912 & $1.2035 \mathrm{E}-02$ & $1.3324 \mathrm{E}-02$ & $1.4828 \mathrm{E}-03$ & $4.4657 \mathrm{E}-02$ & $2.3839 \mathrm{E}-02$ \\
\hline 3946 & $1.1525 \mathrm{E}-02$ & $1.2479 \mathrm{E}-02$ & $1.2054 \mathrm{E}-03$ & $4.1024 \mathrm{E}-02$ & $2.1226 \mathrm{E}-02$ \\
\hline
\end{tabular}

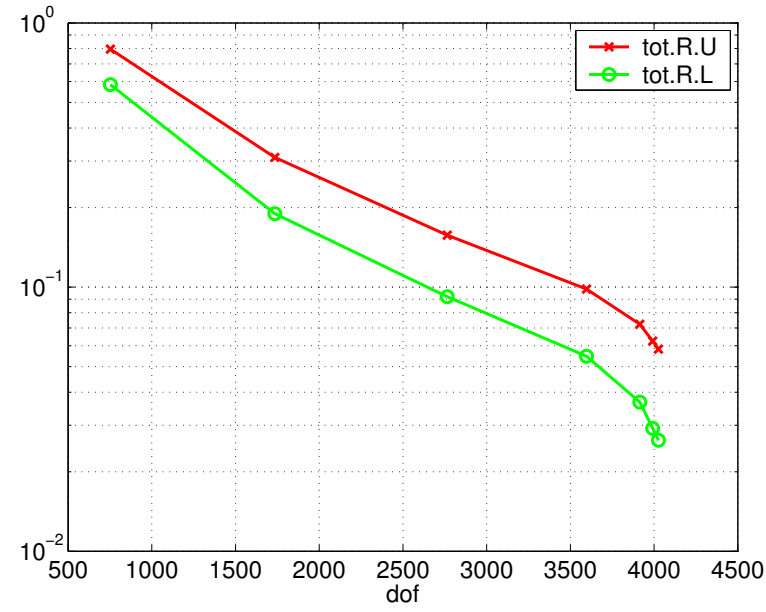

Figure 57: Adaptive problem 1, power-law, Figure 58: Adaptive problem 1, power-law, $r=3.3:$ total residuals during the adaptive process on $\Omega_{2}$ with exact computation of the norm of the residual

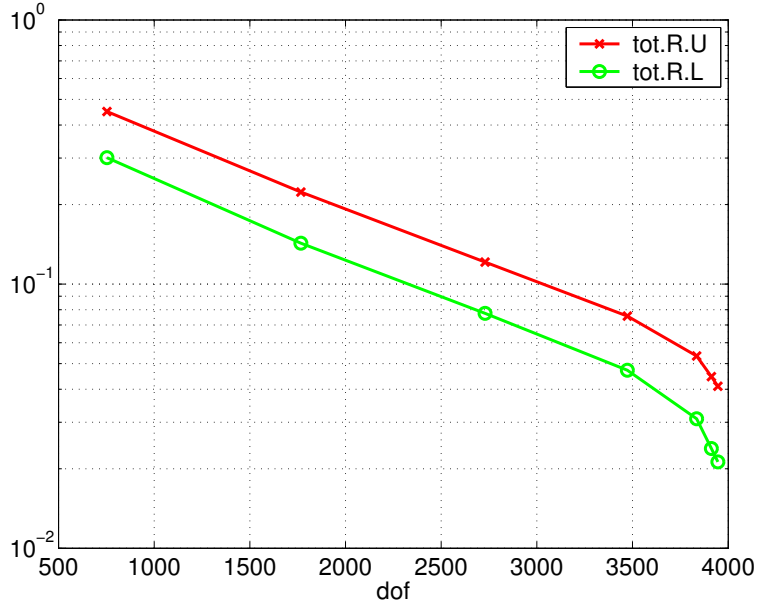

$r=3.3$ : total residuals during the adaptive process on $\Omega_{2}$ with approximate computation of the norm of the residual 


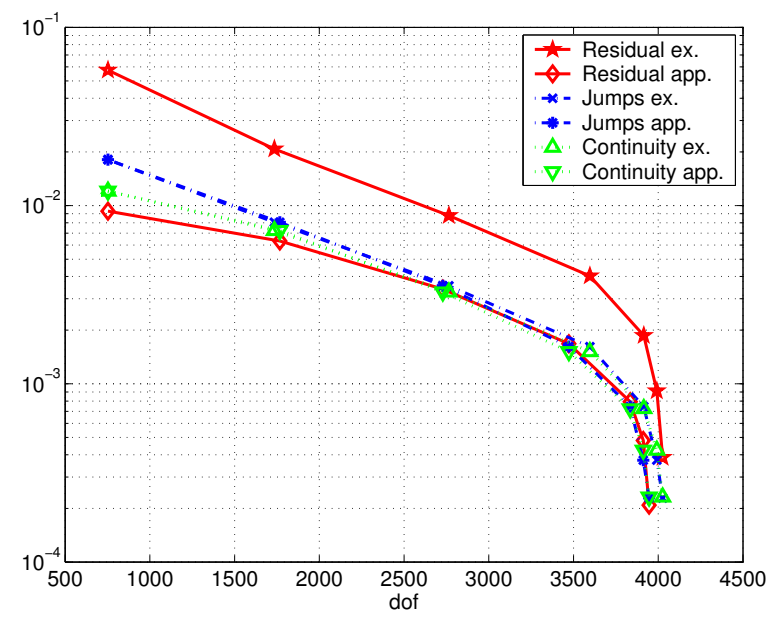

Figure 59: Adaptive problem 1, power-law, Figure 60: Adaptive problem 1, power-law, $r=3.3, \Omega_{2}$, comparison of the maximal val- $r=3.3, \Omega_{2}$, comparison of the mean-values ues of the residuals on the elements

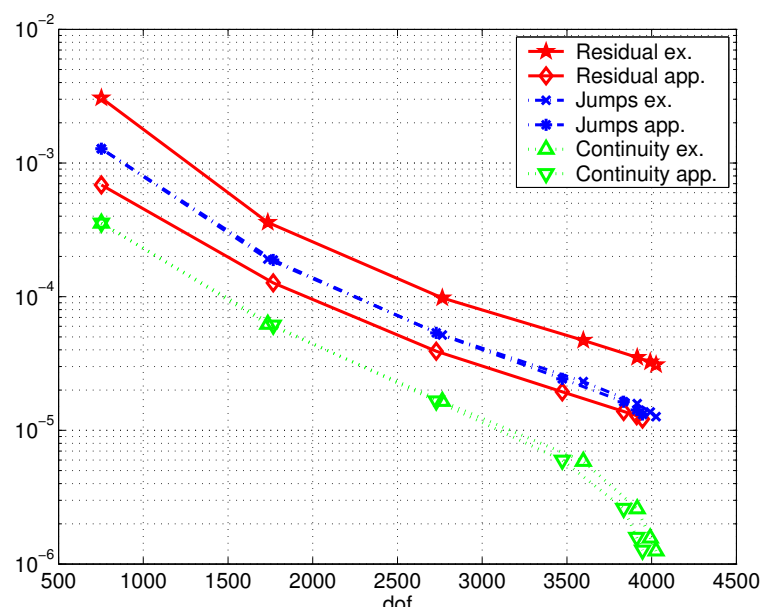

of the residuals on the elements

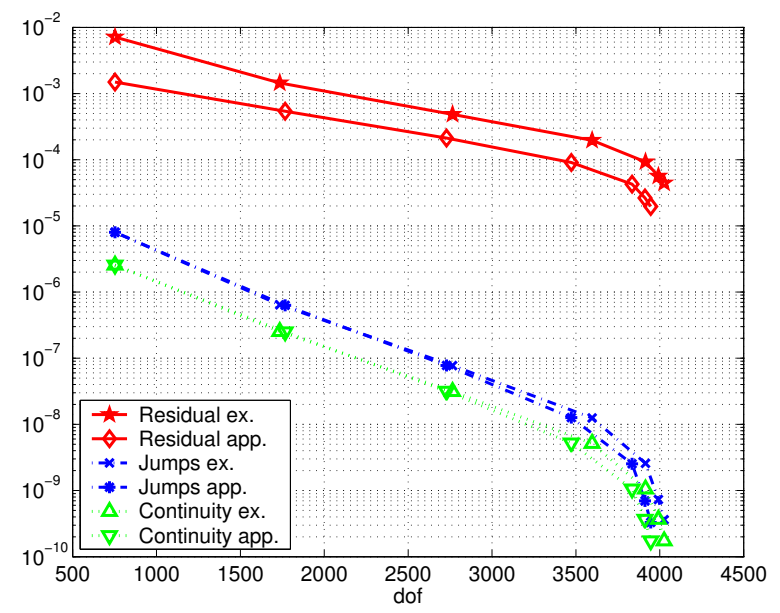

Figure 61: Adaptive problem 1, power-law, $r=3.3, \Omega_{2}$, comparison of the r.m.s. values of the residuals on the elements 


\section{Concluding remarks}

We presented a general framework for energy-norm-based a posteriori error analysis for conforming mixed finite element approximations to quasi-Newtonian flow models. As has been noted in the Introduction, Proposition 13 and Theorem 14 recover a number of known a posteriori bounds from the literature; they also provide new bounds for a very general class of quasi-Newtonian flow models.

When $r=2$, we have $\mathrm{R}_{\mathrm{U}}=\mathrm{R}_{\mathrm{U}}^{\prime}=\mathrm{R}_{\mathrm{L}}=\mathrm{R}_{\mathrm{L}}^{\prime}=\mathrm{s}=\mathrm{g}^{\prime}=2$; then, (1.8) of Theorem 14 collapses to the a posteriori error bound of Barrett and Bao [6] for inf-sup-stable mixed finite element approximations of Carreau-type quasi-Newtonian flows, the linear Stokes problem being a special case [26]. For $r \neq 2$, the bound (1.8) represents a generalization of several earlier results (cf. [4] and [20], in particular).

The validity of Propositions 12 and 13 and of the lower bounds is independent of whether or not the pair of spaces $\left(V_{h}, Q_{h}\right)$ is inf-sup stable in the sense of $(2.1)$ : it is only in the transition from Proposition 13 to Theorem 14 that made use of the bound on $\left\|p_{h}\right\|_{Q}$ from (3.10) which relied on (1.6). Indeed, suppose that problem (1.4), (1.5) has been approximated by the following finite element method: find $\mathbf{u}_{h} \in V_{h}$ and $p_{h} \in Q_{h}$ such that

$$
\begin{array}{rlrl}
a\left(\mathbf{u}_{h}, \mathbf{v}_{h}\right)+b\left(p_{h}, \mathbf{v}_{h}\right) & =\left(\mathbf{f}, \mathbf{v}_{h}\right) & & \forall \mathbf{v}_{h} \in V_{h}, \\
b\left(q_{h}, \mathbf{u}_{h}\right) & =c_{h}\left(q_{h}, p_{h}\right) & \forall q_{h} \in Q_{h},
\end{array}
$$

where $c_{h}(\cdot, \cdot)$ is a bilinear form on $Q_{h} \times Q_{h}$ satisfying $c_{h}\left(q_{h}, q_{h}\right) \geq 0, q_{h} \in Q_{h}$ (the discretisation $(2.2),(2.3)$ being a special case with $c_{h}\left(q_{h}, p_{h}\right)=0$ for all $\left.q_{h} \in Q_{h}\right)$. A number of pressure-stabilised finite element discretisations of (1.4), (1.5) are of this form. It is then easy to see that the bound on $\left\|\mathbf{u}_{h}\right\|_{V}$ from (3.10) still holds irrespective of (2.1), and, if instead of assuming (2.1) we suppose that the sequence $\left(\left\|p_{h}\right\|_{Q}\right)_{h>0}$ is bounded, independent of $h$, then, once again, Theorem 14 follows from Proposition 13 in exactly the same way as before.

In some of our numerical experiments we observed variation of the effectivity indices with the meshsize, which suggests that there is scope for further sharpening of the a posteriori bounds. Nevertheless, in none of the adaptive numerical experiments performed did this lack of robustness lead to unreasonable meshes. In fact, in all cases considered we obtained satisfactory meshes, suggesting that these estimates lead to an acceptable mesh adaptation strategy.

The fact that in some circumstances the quantities tot.R.U and tot.R.L exhibited identical behaviour during the adaptive process while in other cases a smooth diverging trend of the two quantities was observed, is probably due to the different rates of decay of the momentum residuals (R.res and R.jump) and the continuity residual (R.cont). Indeed, when the residuals R.res and R.jump do not decrease as fast as R.cont, as in the Carreau law problem with $r=1.3$, we observe such a diverging behaviour of the upper and lower effectivity indices. 


\section{Acknowledgements}

The first author acknowledges the support of the Istituto Nazionale di Alta Matematica "Francesco Severi" and Italian research project PRIN 2004 "Adattività e avanzamento in tempo nei modelli numerici alle derivate parziali", coordinated by Professor Franco Brezzi.

\section{References}

[1] C. Amrouche and V. Girault, Propriétés fonctionelles d'opérateurs. Application au problème de Stokes en dimension quelconque, Publications du Laboratoire d'Analyse Numérique, n. 90025, Université Pierre et Marie Curie, Paris, 1990.

[2] S. Balay, K Buschelman, V Eijkhout, W.D. Gropp, D. Kaushik, M.G. Knepley, L. Curfman McInnes, B.F. Smith, H. Zhang, PETSc Users Manual, ANL-95/11 - Revision 2.1.5, Argonne National Laboratory, 2004. http://www-unix.mcs.anl.gov/petsc

[3] W. BAO, An economical finite element approximation of generalized Newtonian flows, Comput. Methods Appl. Mech. Engnrg. 191 (2002) 3637-3648.

[4] J. BARAnger AND H. El Amri, Estimateurs a posteriori d'erreur pour calcul adaptif d'écoulements quasi-Newtoniens, RAIRO $M^{2} A N 25$ (1991), 31-48.

[5] J. BARAnger AND K. NAJiB, Analyse numérique des écoulements quasiNewtoniens dont la viscosité obéit à la loi puissance ou la loi de Carreau, Numer. Math. 58 (1990) 35-49.

[6] J.W. Barrett and W. BaO, A priori and a posteriori error bounds for a nonconforming linear finite element approximation of non-Newtonian flow, $M^{2} A N \mathbf{3 2}$ (1998) 843-858.

[7] J.W. BARrett And W.B. Liu, Finite element error analysis of quasi-Newtonian flow obeying the Carreau or power law, Numer. Math. 64 (1993) 433-453.

[8] J.W. BARRETT AND W.B. LiU, Quasi-norm error bounds for finite element approximations of quasi-Newtonian flows, Numer. Math. 68 (1994) 437-456.

[9] J.W. Barrett, J.A. Robson And E. Süli, A posteriori error analysis of mixed finite element approximations to quasi-Newtonian incompressible flows. Technical Report NA-04/13, Oxford University Computing Laboratory, 2004. http://web.comlab.ox.ac.uk/oucl/publications/natr/na-04-13.html

[10] S.C. Brenner And L.R. Scott, The Mathematical Theory of Finite Element Methods, Springer-Verlag, Berlin, 2002. 
[11] C. Carstensen and S.A. Funken, A posteriori error control in low-order finite element discretisations of incompressible stationary flow problems. Math. Comput. 70 (2001) 1353-1381.

[12] P. Clément, Approximation by finite element functions using local regularization, RAIRO Série Rouge Anal. Numér. 9 (1975), R-2, 77-84.

[13] P.G. Ciarlet, The Finite Element Method for Elliptic Problems, North-Holland Publishing Company, Amsterdam, 1978.

[14] Q. Du And M. Gunzburger, Finite element approximation of Ladyzhenskaya model for stationary incompressible viscous flow, SIAM J. Numer. Anal. 27 (1990) $1-19$.

[15] M. Fortin And M. Soulie, A non-conforming piecewise quadratic finite element on triangles. Internat. J. Numer. Methods Engrg., 19 (1983) 505-520.

[16] C.O. Horgan, Korn's inequalities and their applications in continuum mechanics. SIAM Review 37 (1995) 491-511.

[17] B.S. Kirk, J.W. Peterson, R. Stogner, S. Petersen, LibMesh. The University of Texas, Austin, CFDLab and Technische Universität Hamburg, Hamburg. http://libmesh.sourceforge.net.

[18] R. Kouhia And R. Stenberg, A linear nonconforming finite element method for nearly incompressible elasticity and Stokes flow, Comput. Methods Appl. Mech. Engnrg. 124 (1995) 195-212.

[19] P.P. Mosolov and V.P. Myasnikov, A proof of Korn's inequality, Soviet Math. Dokl. 12 (1971) 1618-1622.

[20] C. PADrA, A posteriori error estimators for nonconforming approximation of some quasi-Newtonian flows, SIAM J. Numer. Anal. 34 (1997) 1600-1615.

[21] D. SANDRI, Sur l'approximation numériqui des écoulements quasi-Newtoniens dont la viscosité suit loi puissance ou la loi de Carreau, RAIRO $M^{2} A N 27$ (1993) 131-155.

[22] D. SANDRI, A posteriori error estimators for mixed finite element approximations of a fluid obeying the power law, Comput. Methods Appl. Mech. Engnrg. 166 (1998) 329-340.

[23] G. Simms, Finite element approximation of some nonlinear elliptic and parabolic problems. Ph.D. Thesis. Imperial College London, London 1995.

[24] L.R. Scott And S. Zhang, Finite element interpolation of nonsmooth functions satisfying boundary conditions, Mathematics of Computation 54 (1990) 483-493.

[25] R. Verfürth, A posteriori error estimates for nonlinear problems. Finite element discretizations of elliptic equations, Math. Comput. 62 (1994), 445-475. 
[26] R. Verfürth, A Review of a Posteriori Error Estimation and Adaptive MeshRefinement Techniques, Teubner Verlag and J. Wiley, Stuttgart, 1996. 